Math. Model. Nat. Phenom.

Vol. 7, No. 4, 2012, pp. 99-145

DOI: $10.1051 / \mathrm{mmnp} / 20127408$

\title{
Dynamics of a Reactive Thin Film
}

\author{
P.M.J. Trevelyan ${ }^{1,2}$, A. Pereira ${ }^{1}$, S. Kalliadasis ${ }^{1 *}$ \\ ${ }^{1}$ Department of Chemical Engineering, Imperial College London, London SW7 2AZ, United Kingdom \\ ${ }^{2}$ Division of Mathematics \& Statistics, University of Glamorgan, Pontypridd, CF37 1DL, Wales
}

\begin{abstract}
Consider the dynamics of a thin film flowing down an inclined plane under the action of gravity and in the presence of a first-order exothermic chemical reaction. The heat released by the reaction induces a thermocapillary Marangoni instability on the film surface while the film evolution affects the reaction by influencing heat/mass transport through convection. The main parameter characterizing the reaction-diffusion process is the Damköhler number. We investigate the complete range of Damköhler numbers. We analyze the steady state, its linear stability and nonlinear regime. In the latter case, long-wave models are compared with integral-boundary-layer ones and bifurcation diagrams for permanent solitary wave solutions of the different models are constructed. Time-dependent computations with the integral-boundarylayer models show that the system approaches a train of coherent structures that resemble the solitary pulses obtained in the bifurcation diagrams.
\end{abstract}

Keywords and phrases: thin films, Marangoni effect, chemical reactions

Mathematics Subject Classification: 76E17, 76E30, 76V05

\section{Introduction}

Thin liquid films on a substrate are encountered in numerous applications and problems in engineering, chemistry and biology. As a consequence, numerous studies, both experimental and theoretical, have been devoted to the dynamics of thin films in different settings and configurations for several decades; see e.g. [1], [2] and [3] for reviews. For example, the problem of a film flowing down an inclined plane under the influence of gravity has received considerable attention since the pioneering works of $[4,5]$ and [6] who first provided a systematic exploration of its flow characteristics; see [7] and [8] for reviews.

In many situations of practical interest, a thin film cannot be isolated from its environment and one has to take into account the presence of additional effects and complexities such as concentration/heat effects and chemical reactions taking place in the film. For example, one of the chemical species may be a surfactant changing the surface tension of the liquid/gas interface. In that case, the dynamics of the film can be profoundly affected by the chemical reaction which can give rise to solitary pulses on the surface of the film [9-12]. The influence of chemical reactions has also been investigated in the case of droplets moving in a solution [13] and on a substrate [14]. In this study, we focus on the case of a film falling down a planar substrate under the influence of gravity and in the presence of an exothermic chemical reaction taking place in the bulk. This is the typical situation encountered for example in falling film reactors. In this case, it is important for engineering purposes and process efficiency to control the film

${ }^{*}$ Corresponding author. E-mail: s.kalliadasis@imperial.ac.uk 
characteristics. In particular, the film surface area and the time of contact between the liquid and the gas are often key parameters for chemical reactions involving reactants from the liquid and gas phases. Also, in several industrial applications, falling films involving specific reactants facilitate the absorption of gas components. Examples are reactions between $\mathrm{SO}_{2}$ (solute gas) and $\mathrm{KOH}$ (reactant in liquid), $\mathrm{CO}_{2}$ and Hydroxides, $\mathrm{Cl}_{2}$ and $\mathrm{FeCl}_{2}$ [15]. In these cases, the quantity of interest is the adsorption rate which is influenced by several parameters like reaction kinetics, species diffusion and liquid density and viscosity. One of the goals of this study is to examine how the chemical reaction will affect the liquid-gas surface area mainly through the influence of temperature on the physical properties of the liquid. Two aspects, in particular, are considered: the linear stability of a film of uniform thickness and the nonlinear dynamics after destabilization, in particular the emergence and dynamics of solitary waves on the film surface.

In order to identify the basic dynamic characteristics of the coupling between the fluid flow and the reaction-diffusion process, the kinetics here is kept as simple as possible. The chemical reaction is a first-order one in which the reactant is assumed to be provided through the film surface by absorption into the liquid.

To model the nonlinear dynamics of reactive films, several approaches are possible. The first one is based on a long-wave expansion of all variables; it is valid close to criticality but fails to account for the nonlinear regime. Because of that, we favor a second approach, based on a weighted residuals technique with a Galerkin projection. As far as the chemical reaction is concerned, we consider the cases of small, moderate and large reaction rates. For large reaction rates we demonstrate the presence of a concentration boundary layer at the free surface which is resolved via matched asymptotics.

The case of a slow reaction has already been investigated in previous studies by Trevelyan and Kalliadasis $[16,17]$, while in this study we examine both fast and slow reaction rates. The basic differences between these studies and the present one are: (i) in $[16,17]$ the different dimensionless groups, such as Reynolds, Weber and Damköhler numbers, were varied independently from each other corresponding to changing the liquid and the reaction-diffusion process. While varying independently the different parameters is something that is frequently done in the thin-films literature, it is more appropriate to introduce a parametrization that distinguishes clearly between the flow, the physical properties of the gas-liquid system and the reaction-diffusion process, as in experiments; (ii) consideration of the Orr-Sommerfeld eigenvalue problem; (iii) non-zero free-surface Biot number.

The paper is organized as follows. In $\S 2$ we define the problem and give the governing equations and dimensionless groups. In $\S 3$ and $\S 4$, we examine the steady state of the system and its linear stability. In $\S 5$ we derive the long-wave models while $\S 6$ is devoted to the weighted residuals ones. In $\S 7$ we present solitary wave solutions and their bifurcation diagrams and in $\S 8$ time-dependent computations. We conclude in $\S 9$.

\section{Physical problem and formulation}

Figure 1 shows the problem definition. We consider a thin liquid film falling under the influence of gravity down an inclined planar substrate forming an angle $\beta$ with the horizontal direction. The liquid has viscosity $\mu$, density $\rho$, constant pressure heat capacity $c_{p}$, thermal conductivity $\lambda$ and surface tension $\sigma$. With the exception of surface tension, the liquid properties are assumed to be constant and independent of temperature (applicable if the temperature variations in the film are small). We also assume that the liquid is non-volatile so that evaporation effects can be neglected. We restrict our attention to the two-dimensional dynamics of the film. A Cartesian coordinate system $(x, y)$ is chosen so that $x$ is in the direction parallel to the substrate and $y$ is the outward-pointing coordinate normal to the substrate. The wall is then located at $y=0$ and the free surface at $y=h(x, t)$. 


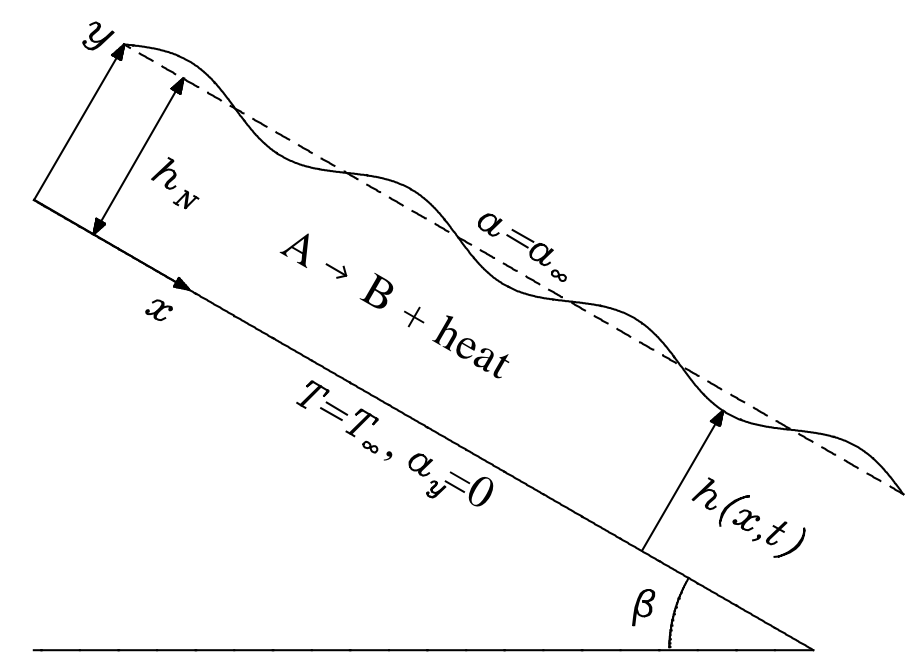

FIgURE 1. Sketch of the profile geometry for an inclined falling film in the presence of a first-order exothermic chemical reaction $\mathrm{A} \rightarrow \mathrm{B}+$ heat. $h(x, t)$ is the local film thickness, $h_{\mathrm{N}}$ is the Nusselt flat film thickness and $\beta$ is the inclination angle with respect to the horizontal direction.

\subsection{Hydrodynamics}

The governing equations for the fluid flow are the mass conservation and Navier-Stokes equations,

$$
\begin{aligned}
\nabla \cdot \boldsymbol{u} & =0 \\
\rho\left(\boldsymbol{u}_{t}+(\boldsymbol{u} \cdot \boldsymbol{\nabla}) \boldsymbol{u}\right) & =-\nabla p+\nabla \cdot \boldsymbol{\tau}+\rho \boldsymbol{g}
\end{aligned}
$$

where $\boldsymbol{\nabla}=(\partial / \partial x, \partial / \partial y)$ is the gradient operator on the $(x, y)$ plane, $\boldsymbol{u}=(u, v)$ is the fluid velocity vector, $\boldsymbol{g}=g(\sin \beta,-\cos \beta)$ with $g$ the magnitude of the gravitational acceleration, $p$ is the fluid pressure and $\boldsymbol{\tau}=\mu\left[(\boldsymbol{\nabla u})+(\boldsymbol{\nabla u})^{t}\right]$ is the deviatoric stress tensor. Moreover, in equation (2.1) and throughout the paper, partial derivatives are denoted by subscripts (i.e. $\boldsymbol{u}_{t}$ refers to $\partial \boldsymbol{u} / \partial t$ ).

On the wall we have the no-slip boundary condition,

$$
\boldsymbol{u}=\mathbf{0} \quad \text { on } \quad y=0
$$

while on the free surface we have the kinematic boundary condition and the normal and tangential stress balances, respectively,

$$
\begin{aligned}
h_{t}+(\boldsymbol{u} \cdot \boldsymbol{\nabla})(h-y) & =0 \quad \text { on } \quad y=h \\
p_{\infty}-p+(\boldsymbol{\tau} \cdot \mathbf{n}) \cdot \mathbf{n} & =2 \sigma K(h) \quad \text { on } \quad y=h \\
(\boldsymbol{\tau} \cdot \mathbf{n}) \cdot \mathbf{t} & =\nabla_{s} \sigma \cdot \mathbf{t} \quad \text { on } \quad y=h,
\end{aligned}
$$

where $\mathbf{n}$ and $\mathbf{t}$ are unit vectors, normal (outward pointing) and tangential to the interface, respectively, defined from $\mathbf{n}=N^{-1 / 2}\left(-h_{x}, 1\right), \mathbf{t}=N^{-1 / 2}\left(1, h_{x}\right)$ where $N=1+h_{x}^{2} . K(h)=-(1 / 2) \nabla \cdot \mathbf{n}$ is the curvature of the interface and $\nabla_{s}=(\mathbf{I}-\mathbf{n} \otimes \mathbf{n}) \cdot \boldsymbol{\nabla}$ is the surface gradient operator with $\mathbf{I}$ the $2 \times 2$ unitary matrix. $p_{\infty}$ is the pressure of the ambient gas phase which can be absorbed into a redefined pressure and hence without loss of generality can be taken as zero.

The coupling between hydrodynamics and chemistry is achieved through the Marangoni effect in the right-hand-side of the tangential stress balance (2.3c). The Marangoni effect also influences the normal stress balance but this is typically a higher-order effect compared to the influence on the tangential stress. 


\subsection{The reaction-diffusion process}

The ambient gas phase contains a species A that is absorbed into the liquid at the interface. Once species A enters the liquid it undergoes a simple first-order decay,

$$
\mathrm{A} \rightarrow \mathrm{B}+\text { heat }
$$

with both reactant A and product B assumed to be passive, i.e. they have no effect on the flow field (via e.g. changing the density and/or the surface tension). Note that although few interesting reactions are truly first order, many can appear to be so under certain circumstances, for example in the presence of an excess of one of the reactants.

The heat generated by the reaction, induces a thermocapillary Marangoni effect, which in turn, affects the free-surface, fluid flow, and hence adsorption properties of the film. The thermocapillary effect is modeled by assuming a linear dependence of the surface tension on temperature,

$$
\sigma=\sigma_{\infty}-\gamma\left(T-T_{\infty}\right),
$$

where $\sigma_{\infty}$ is the surface tension at the far field gas temperature $T_{\infty} . \gamma$ is positive in most cases corresponding to the frequently encountered property of liquids that their surface tension decreases as the temperature increases. However, there are several liquids which are known to display the opposite behavior [18].

The governing equations for the reaction-diffusion process are the continuity of species $\mathrm{A}$ and the energy equation,

$$
\begin{aligned}
a_{t}+(\boldsymbol{u} \cdot \boldsymbol{\nabla}) a & =D \nabla^{2} a-a k(T) \\
\rho c_{p}\left[T_{t}+(\boldsymbol{u} \cdot \boldsymbol{\nabla}) T\right] & =\lambda \nabla^{2} T+a q_{0} k(T),
\end{aligned}
$$

where $a$ and $T$ denote the concentration of A and fluid temperature, respectively, $D$ is the molecular diffusivity of $\mathrm{A}$ and $q_{0}$ is the heat of reaction which is assumed to be independent of temperature. $k(T)$ is the part of the reaction rate that depends on the temperature following the Arrhenius law,

$$
k(T)=k_{0} e^{-E_{a} / R T},
$$

where $k_{0}$ is the frequency (or pre-exponential factor), $E_{a}$ is the activation energy and $R$ is the ideal gas constant.

The wall is an impermeable boundary so that there is no mass flux though the wall:

$$
a_{y}=0 \quad \text { on } \quad y=0 .
$$

The wall is also held at a constant temperature which, for simplicity, is taken to be the same as that of the far field gas temperature $T_{\infty}$ :

$$
T=T_{\infty} \quad \text { on } \quad y=0 .
$$

Hence the wall is a 'cold boundary' having a cooling effect on the film.

On the free surface, we assume that the concentration of A remains constant,

$$
a=a_{\infty} \quad \text { on } \quad y=h .
$$

This is effectively the case if an unlimited supply of A is available from the gas and the mass-transport coefficient there is large so that the concentration of $\mathrm{A}$ in the gas is constant, and if an equilibrium relationship between the concentration of $\mathrm{A}$ in the gas and in the liquid near the surface (in the form of Henry's law for example) is assumed. Note, however, that the equilibrium relationship usually depends on temperature but in the present study we assume this to be a higher-order effect given the range 
of temperatures considered. Finally, on the free surface we have Newton's law of cooling in which the temperature difference between the interfacial fluid temperature and that of the surrounding gas is balanced by the heat flux normal to the interface,

$$
\lambda \nabla T \cdot \mathbf{n}=-\alpha_{s}\left(T-T_{\infty}\right) \quad \text { on } \quad y=h,
$$

with $\alpha_{s}$ the heat transfer coefficient between the liquid and the gas.

Some typical physical parameters are given in table 1 (see e.g. [19]). To keep the problem as simple as possible this study will focus on the already established regime away from the inlet where flow, concentration and heat are fully developed, and thus periodic boundary conditions are imposed in the streamwise direction. This in turn allows for the base state solutions to be spatially uniform in the streamwise direction, which would not be valid near the inlet.

\subsection{Applications}

Although we shall keep this study as general as possible we note that there are several situations to which it could be applicable. As the reaction is a first-order one could consider for example the case of catalytic reactions or acid base reactions. Here we discuss the latter. More specifically, we consider two acid-base reactions, a fast one and a slow one, in which the gas contains the acid and the liquid contains the base, and then assume that the base is in excess in the liquid and the acid is in excess in the gas.

First let us take a fast acid-base reaction. Consider hydrochloric acid $(\mathrm{HCl})$ in gaseous form and a diluted aqueous solution of sodium hydroxide $(\mathrm{NaOH})$ falling down the wall. By assuming that the concentration of $\mathrm{NaOH}$ is sufficiently large compared to the concentration of $\mathrm{HCl}$ being absorbed at the interface, then the exothermic reaction $\mathrm{NaOH}+\mathrm{HCl} \rightarrow \mathrm{NaCl}+\mathrm{H}_{2} \mathrm{O}$ could be treated as a first order reaction, namely $\mathrm{A} \rightarrow \mathrm{B}+$ heat, where $\mathrm{A}$ represents $\mathrm{HCl}$ and $\mathrm{B}$ represents $\mathrm{NaCl}$. For this to be a truly first order reaction the reaction rate should only depend on the concentration of species $\mathrm{A}$. We have assumed that the concentration of $\mathrm{NaOH}$ is in excess in the liquid, and the concentration of $\mathrm{HCl}$ is in excess in the gas. Both these conditions could not be met in the same medium, but this is not a problem here as we have two different mediums and so there is no contradiction. A second problem with theses assumptions is that they becomes less valid as the length of the wall increases as more and more $\mathrm{NaOH}$ would be consumed and thus the reaction would then be slower further downstream and faster upstream. In addition, making the already thin film thinner would also amplify this effect. However, these problems can easily be avoided by making the ratio of the concentration of $\mathrm{NaOH}$ say $10^{4}$ times larger than the anticipated level of $\mathrm{HCl}$ absorbed on to the interface, so that $\mathrm{NaOH}$ is in such excess that it is reasonable to assume that its concentration is constant. Additional concerns that one might have about this reaction is that one of the products is the salt $\mathrm{NaCl}$ which can act as a weak surfactant inducing a weak solutal Marangoni effect, however, we anticipate that the thermal Marangoni effect associated with the (moderate) reaction exothermicity would dominate the resulting instability. Furthermore, the presence of different species of different densities could induce a variety of buoyancy instabilities, however, as we are considering the film thickness to be thin, the presence of such buoyancy instabilities can also be reduced.

Secondly, let us take a slow acid-base reaction. Carbon dioxide sequestration is a currently a very active area of research, in which gaseous $\mathrm{CO}_{2}$ is taken from the air, absorbed into water and then sinks under gravity. A small fraction of the aqueous $\mathrm{CO}_{2}$ slowly reacts with the water to form carbonic acid $\mathrm{H}_{2} \mathrm{CO}_{3}$. This reaction is only very weakly exothermic, however, several other reactions involving $\mathrm{H}_{2} \mathrm{CO}_{3}$ can follow from this, including exothermic reactions. If we ignore the additional reactions which may follow, then it is reasonable to consider this as a slow first-order exothermic reaction. Admittedly, such a system would be an inefficient way of removing $\mathrm{CO}_{2}$ from a gas, however, there are some involved modifications making our system more complicated, by e.g. including additional reactants, which lead to improved efficiency. For example [20] included additional reactions involving sodium carbonate and bicarbonate, but the emphasis of this study was on buoyancy instabilities. 
TABLE 1. Typical physical constants

\begin{tabular}{cc}
\hline$g$ & $9.81 \mathrm{~m} \mathrm{~s}^{-2}$ \\
$R$ & $8.314 \mathrm{~J} \mathrm{~mol}^{-1} \mathrm{~K}^{-1}$ \\
$c_{P}$ & $4.186 \times 10^{3} \mathrm{~J} \mathrm{~kg}^{-1} \mathrm{~K}^{-1}$ \\
$\rho$ & $10^{3} \mathrm{~kg} \mathrm{~m}^{-3}$ \\
$\nu$ & $10^{-6} \mathrm{~m}^{2} \mathrm{~s}^{-1}$ \\
$D$ & $10^{-9} \mathrm{~m}^{2} \mathrm{~s}^{-1}$ \\
$\lambda$ & $0.6 \mathrm{~J} \mathrm{~m}^{-1} \mathrm{~s}^{-1} \mathrm{~K}^{-1}$ \\
$\sigma$ & $7 \times 10^{-2} \mathrm{~N} \mathrm{~m}^{-1}$ \\
$\gamma$ & $1.7 \times 10^{-4} \mathrm{~N} \mathrm{~m} \mathrm{~K}^{-1}$ \\
$T_{\infty}$ & $293 \mathrm{~K}^{-1}$ \\
$a_{\infty}$ & $10^{-3} \mathrm{to} \mathrm{mol} \mathrm{m}^{-3}$ \\
$h_{0}$ & $10^{-5} \mathrm{to}^{-3} \mathrm{~m}^{-1}$ \\
$E_{a}$ & $2.5 \times 10^{3} \mathrm{~J} \mathrm{~mol}^{-1}$ \\
$k_{0}$ & $10^{-3}{\mathrm{to} 10^{6} \mathrm{~s}^{-1}}$ \\
$q_{0}$ & $10^{5} \mathrm{~J} \mathrm{~mol}^{-1}$ \\
$\alpha_{s}$ & $10^{2} \mathrm{~W} \mathrm{~m}^{-2} \mathrm{~K}^{-1}$ \\
\hline
\end{tabular}

\subsection{Scalings and non-dimensionalization}

In the absence of a reaction, i.e. if $k_{0}=0$, the coupled system of equations (2.1-2.7) has a trivial solution corresponding to the plane-parallel base state,

$$
\begin{gathered}
h=h_{\mathrm{N}}, u=\frac{g \sin \beta}{2 \nu}\left(2 h_{\mathrm{N}} y-y^{2}\right), v=0, p=p_{\infty}+\rho\left(h_{\mathrm{N}}-y\right) g \cos \beta, \\
a=a_{\infty}, \quad T=T_{\infty}
\end{gathered}
$$

where (2.8a) is the Nusselt flat-film solution corresponding to a balance between viscous and gravitational forces. Equation $(2.8 \mathrm{~b})$ is only valid in the absence of a chemical reaction. The flow is driven by the hydrodynamics and hence the length/time scales and therefore the velocity scales should be based on the hydrodynamics. Using both the dimensional Nusselt flat film thickness $h_{\mathrm{N}}$ and the viscous-gravity length and time scales,

$$
l_{\nu}=\frac{\nu^{2 / 3}}{(g \sin \beta)^{1 / 3}}, \quad t_{\nu}=\frac{\nu^{1 / 3}}{(g \sin \beta)^{2 / 3}},
$$

we employ the non-dimensionalization

$$
\begin{gathered}
(x, y, h)=h_{\mathrm{N}}(\bar{x}, \bar{y}, \bar{h}), \quad t=\frac{t_{\nu} l_{\nu}}{h_{\mathrm{N}}} \bar{t}, \quad(u, v)=\frac{h_{\mathrm{N}}^{2}}{t_{\nu} l_{\nu}}(\bar{u}, \bar{v}), \quad p=p_{\infty}+\rho \frac{l_{\nu} h_{\mathrm{N}}}{t_{\nu}^{2}} \bar{p} \\
a=a_{\infty}(1+\mathcal{D} \bar{a}), \quad T=T_{\infty}(1+\mathcal{D} \phi \bar{T}),
\end{gathered}
$$

where the dimensionless groups $\mathcal{D}$ and $\phi$ associated with the reaction-diffusion process will be defined shortly (the introduction of these parameters in the non-dimensionalization for $a$ and $T$ in $(2.9 \mathrm{~b})$ is done purely on the basis that it simplifies the subsequent algebra).

The dimensionless bulk equations then in (2.1) and (2.4) are

$$
\begin{aligned}
u_{x}+v_{y} & =0 \\
3 \operatorname{Re}\left(u_{t}+u u_{x}+v u_{y}\right) & =-p_{x}+u_{x x}+u_{y y}+1 \\
3 \operatorname{Re}\left(v_{t}+u v_{x}+v v_{y}\right) & =-p_{y}+v_{x x}+v_{y y}-\cot \beta \\
3 \operatorname{ReSc}\left(a_{t}+u a_{x}+v a_{y}\right) & =a_{x x}+a_{y y}-(1+\mathcal{D} a) e^{\frac{\mathcal{E D} \phi T}{1+\mathcal{D} \phi T}} \\
3 \operatorname{RePr}\left(T_{t}+u T_{x}+v T_{y}\right) & =T_{x x}+T_{y y}+(1+\mathcal{D} a) e^{\frac{\mathcal{E D} \phi T}{1+\mathcal{D} \phi T}}
\end{aligned}
$$


where bars have been dropped for convenience. The dimensionless wall boundary conditions in $(2.2)$ and (2.6) are

$$
u=v=a_{y}=T=0 \quad \text { on } \quad y=0
$$

and the dimensionless free-surface boundary conditions in (2.3) and (2.7) are:

$$
\begin{gathered}
p+(\mathrm{We}-\mathcal{M T}) N^{-3 / 2} h_{x x}=2 N^{-1}\left(v_{y}-h_{x}\left(u_{y}+v_{x}\right)+u_{x} h_{x}^{2}\right) \quad \text { on } y=h \\
\left(u_{y}+v_{x}\right)\left(1-h_{x}^{2}\right)+2 h_{x}\left(v_{y}-u_{x}\right)=-\mathcal{M}\left(T_{x}+h_{x} T_{y}\right) N^{1 / 2} \quad \text { on } \quad y=h \\
h_{t}+u h_{x}-v=a=T_{y}-h_{x} T_{x}+B T N^{1 / 2}=0 \quad \text { on } y=h .
\end{gathered}
$$

It should be noted that with the introduction of the parameter $\mathcal{D}$ in $(2.9 \mathrm{~b})$, in the absence of a chemical reaction i.e. $\mathcal{D}=0, a=a_{\infty}$ and $T=T_{\infty}$, as expected. On the other hand, the introduction of the parameter $\phi$ in $(2.9 \mathrm{~b})$ makes the sink/source terms of the concentration/energy equations (2.10d,e), respectively, have the same magnitude.

The system of equations in (2.10-2.12) is therefore governed by the following dimensionless groups and parameters

$$
\operatorname{Re}=\frac{\bar{h}_{\mathrm{N}}^{3}}{3}, \quad \mathrm{We}=\frac{\Gamma}{\bar{h}_{\mathrm{N}}^{2}}, \quad B=\operatorname{Bi} \bar{h}_{\mathrm{N}}, \quad \mathcal{D}=\operatorname{Da} \bar{h}_{\mathrm{N}}^{2}
$$

corresponding to the Reynolds, Weber, film Biot and film Damköhler numbers ('film parameters' are based on $h_{\mathrm{N}}$ ), respectively, written explicitly in terms of the dimensionless Nusselt flat film thickness, $\bar{h}_{\mathrm{N}}=h_{\mathrm{N}} / l_{\nu}$, a key parameter of the problem, and

$$
\operatorname{Pr}=\rho c_{p} \frac{\nu}{\lambda}, \quad \mathrm{Sc}=\frac{\nu}{D}, \quad \mathcal{M}=\operatorname{MaDa} \phi
$$

corresponding to the Prandtl, Schmidt and 'composite' Marangoni numbers, respectively. The products RePr and ReSc are the heat/mass transport Péclet numbers which measure the relative importance of conduction/diffusion over convection, respectively. Finally, we have

$$
\mathrm{Bi}=\frac{\alpha_{s} l_{\nu}}{\lambda}, \mathrm{Da}=\frac{k_{0} l_{\nu}^{2}}{D} e^{-\mathcal{E}}, \Gamma=\frac{\sigma_{\infty} t_{\nu}}{\rho \nu l_{\nu}}, \mathrm{Ma}=\frac{\gamma T_{\infty} t_{\nu}}{\rho \nu l_{\nu}}, \mathcal{E}=\frac{E_{a}}{R T_{\infty}}, \phi=\frac{D q_{0} a_{\infty}}{\lambda T_{\infty}}
$$

corresponding to the Biot, Damköhler, Kapitza, Marangoni, Zeldovich (dimensionless activation energy) and reaction exothermicity (dimensionless heat of reaction) numbers, respectively (Bi, Da, and Ma are based on $l_{\nu}$ ). Note that the Marangoni number in both normal and tangential stress boundary conditions $(2.12 \mathrm{a})$ and $(2.12 \mathrm{~b})$, appears always as a product with the Damköhler number and the reaction exothermicity through the modified Marangoni number $\mathcal{M}$. Hence, the Marangoni effect is absent, as expected, in the absence of a chemical reaction, i.e. $\mathrm{Da}=0$, or for an isothermal chemical reaction, i.e. $\phi=0$, or simply for a fluid whose surface tension is not a function of temperature, i.e. Ma $=0$.

The Damköhler number in (2.13c) expresses the relative importance of the diffusive time scale based on $l_{\nu}, \tau_{D}^{l_{\nu}} \sim l_{\nu}^{2} / D$ (time scale for diffusion with the flow - viscous transport), to the chemical time scale $\tau_{c} \sim 1 / k_{0}: \mathrm{Da} \sim k_{0} l_{\nu}^{2} / D \sim \tau_{D}^{l_{\nu}} / \tau_{c}$. On the other hand, the film Damköhler number in (2.13a) expresses the relative importance of the diffusive time scale based on $h_{\mathrm{N}}, \tau_{D}^{h_{\mathrm{N}}} \sim h_{\mathrm{N}}^{2} / D$ (time scale for diffusion into the film) to the chemical time scale $\tau_{c}: \mathcal{D}=\operatorname{Da} h_{\mathrm{N}}^{2} / l_{\nu}^{2} \sim k_{0} h_{\mathrm{N}}^{2} / D \sim \tau_{D}^{h_{\mathrm{N}}} / \tau_{c}$.

As pointed out in $\S 1$, in the studies of $[16,17]$ of a vertical reactive falling film (and in the absence of interfacial heat transfer effects), the different dimensionless groups such as $R e$, We and $\mathcal{D}$ were varied independently from each other corresponding to changing the liquid and the reaction-diffusion process. On the other hand, the set of dimensionless groups and parameters in (2.13) isolates the dependence on the dimensionless Nusselt flat film thickness $\bar{h}_{\mathrm{N}}$ and the physical properties of the problem. Indeed, Pr, Sc, Bi, Da, $\Gamma$, Ma, $\mathcal{E}, \phi$ and $\mathcal{M}$ are all independent of $\bar{h}_{\mathrm{N}}$ and they depend only on the physical properties 
of the liquid-gas system and the reaction-diffusion process, unlike Re, We, $B$ and $\mathcal{D}$ which depend on $\bar{h}_{\mathrm{N}}$ - these groups have been parametrized in terms of $\bar{h}_{\mathrm{N}}$ and the parameters $\Gamma$, Bi and Da which are independent of $\bar{h}_{\mathrm{N}}$. The system is, therefore, governed by the inclination angle $\beta, \bar{h}_{\mathrm{N}}, \mathrm{Pr}, \mathrm{Sc}, \mathrm{Bi}, \mathrm{Da}, \Gamma$, $\mathrm{Ma}, \mathcal{E}$ and $\phi$. Hence, for a fixed $\beta$ and given liquid-gas system and reaction-diffusion process, the only free parameter is $\bar{h}_{\mathrm{N}}$ or equivalently Re which is a flow control parameter, a rather substantial simplification. Indeed, in actual experiments it would be easier to fix the liquid-gas system and the reaction-diffusion process and vary the film thickness via changing the flow rate. On the other hand, for a fixed $\beta$ and given liquid-gas system, the free parameters are $\bar{h}_{\mathrm{N}}$, Da, $\mathcal{E}$ and $\phi$. Hence, the parametrization employed here distinguishes clearly between the flow, the physical properties of the gas-liquid system and the reaction-diffusion parameters.

\section{Flat-film solution}

As pointed out in $\S 2.4$, the plane-parallel base state consists of $(2.8 \mathrm{a})$, the Nusselt flat film solution, which in dimensionless variables is given by $h \equiv 1, u \equiv y-y^{2} / 2, v \equiv 0, p \equiv(1-y) \cot \beta$ and $(2.8 \mathrm{~b})$ valid only in the absence of a chemical reaction. In the presence of a chemical reaction, the flat film concentration and temperature solutions are more involved. Indeed, in this case one needs to solve the flat film concentration and energy equations,

$$
a_{y y}=(1+\mathcal{D} a) e^{\frac{\mathcal{E} \mathcal{D} \phi T}{1+\mathcal{D} \phi T}}, \quad T_{y y}=-(1+\mathcal{D} a) e^{\frac{\mathcal{E} \mathcal{D} \phi T}{1+\mathcal{D} \phi T}}
$$

subject to the boundary conditions

$$
\begin{gathered}
a_{y}=T=0 \quad \text { on } \quad y=0, \\
a=T_{y}+B T=0 \quad \text { on } \quad y=1 .
\end{gathered}
$$

In general, system (3.1) must be solved numerically. Using finite differences the solutions to $a$ and $T$ can easily be obtained. In figure 2 typical flat film concentration/temperature solutions are illustrated using the parameter values $\mathrm{Re}=1$ and $\mathrm{Bi}=0.1$ with $\mathrm{Da}=0.1$ or $\mathrm{Da}=100$. For large $\mathcal{D}$ we find that there is a boundary layer in $a$ near the free surface, as illustrated in figure 2(a), whilst figure 2(b) shows the existence of a boundary layer in $T_{y}$ near the free surface but not in $T$ ( $T$ does not vary rapidly near the surface but $T_{y}$ does).

Although system (3.1) has been solved numerically, analytical solutions are possible in the limits of $\mathcal{D} \rightarrow 0$ and $\mathcal{D} \rightarrow \infty$. The same limits will also be exploited in the linear stability of the flat-film solution and the development of different models to describe the nonlinear regime. Note that due to the scaling $\mathcal{D}=\tau_{D}^{h_{\mathrm{N}}} / \tau_{c}$ given in $\S 2.4$, the small-D limit implies that the reaction rate is slow compared to diffusion into the film. This can be achieved either by a small $k_{0}$ or by a small $h_{\mathrm{N}}^{2} / D$ which for fixed $D$ is equivalent to small $h_{\mathrm{N}}$ (the rate by which species A diffuses is infinitely fast when the length over which it diffuses becomes infinitesimally small). On the other hand, the large- $\mathcal{D}$ limit implies that the reaction rate is fast compared to diffusion into the film. In the following we shall refer to the small/large- $\mathcal{D}$ limits as the 'slow/fast chemical reaction' cases, respectively (with the understanding that the reaction is slow/fast compared to diffusion).

\subsection{Slow reaction}

The assumption $\mathcal{D} \ll 1$ converts the highly nonlinear problem in (3.1) to solvable perturbation problems. By expanding the Arrhenius term up to and including terms of $\mathcal{O}\left(\mathcal{D}^{2}\right)$, the equations in (3.1a) become:

$$
\begin{aligned}
& a_{y y}=1+\mathcal{D}(a+\mathcal{E} \phi T)+\frac{1}{2} \mathcal{D}^{2} \mathcal{E} \phi T(2 a+(\mathcal{E}-2) \phi T)+\mathcal{O}\left(\mathcal{D}^{3}\right) \\
& T_{y y}=-\left[1+\mathcal{D}(a+\mathcal{E} \phi T)+\frac{1}{2} \mathcal{D}^{2} \mathcal{E} \phi T(2 a+(\mathcal{E}-2) \phi T)\right]+\mathcal{O}\left(\mathcal{D}^{3}\right)
\end{aligned}
$$




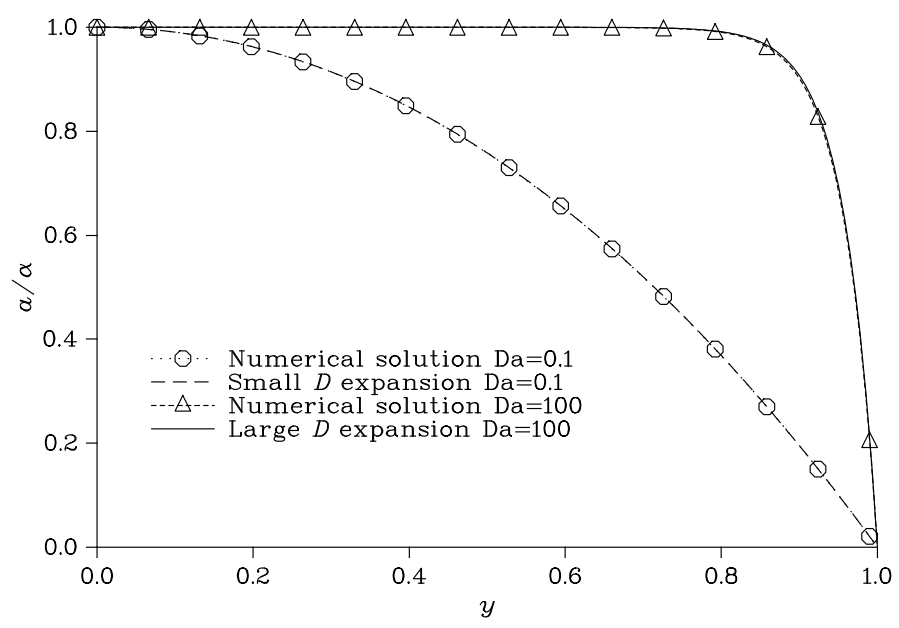

(a)

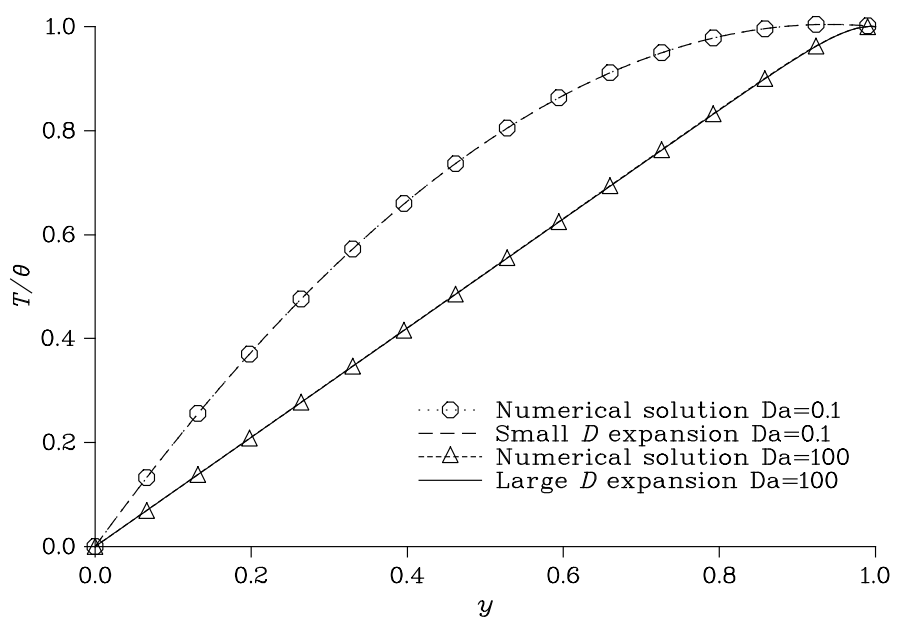

(b)

FiguRE 2. Comparisons of flat-film concentration and temperature profiles obtained from the small/large $\mathcal{D}$ asymptotics with the exact flat-film solutions. The parameter values are: $\mathrm{Re}=1$ and $\mathrm{Bi}=0.1$ (a) Flat-film concentration profiles normalized with the wall concentration $\alpha$. (b) Flat-film temperature profiles normalized with the interfacial temperature $\theta$.

Expanding now $a$ and $T$ up to and including terms of $\mathcal{O}\left(\mathcal{D}^{2}\right)$ and equating coefficients, the above equations with the boundary conditions (3.1b,c) can be solved to yield $a=\left.a_{F}^{s}\right|_{h=1}+\mathcal{O}\left(\mathcal{D}^{3}\right)$ and $T=\left.T_{F}^{s}\right|_{h=1}+\mathcal{O}\left(\mathcal{D}^{3}\right)$ where the functions $a_{F}^{s}$ and $T_{F}^{s}$ are given in Appendix A. Note that the $\mathcal{O}\left(\mathcal{D}^{2}\right)$ concentration terms are given for completeness: due to the presence of $\mathcal{D} a$ in the energy equation $(2.10 \mathrm{e})$, we only need the $\mathcal{O}(\mathcal{D})$ terms of the concentration (they affect the temperature up to and including $\mathcal{O}\left(\mathcal{D}^{2}\right)$ ).

\subsection{Fast reaction}

When the reaction is fast compared to diffusion, i.e. $\mathcal{D} \gg 1$, it consumes the reactant quickly leaving no reactant below a boundary layer near the surface. The formation of this boundary layer is evident when we consider the trivial solution, $a=-\mathcal{D}^{-1}$, of the mass transport equation: it satisfies the wall boundary condition $a_{y}=0$, but it fails to satisfy the surface boundary condition $a=0$ and hence the presence of 
a boundary layer near the surface is required. Consider

$$
a=(\widehat{a}-1) \mathcal{D}^{-1} \quad \text { and } \quad y=1-\delta Y,
$$

where $\delta$ is the thickness of the boundary layer. The mass transport equation then becomes

$$
0=\widehat{a}_{Y Y}-\delta^{2} \mathcal{D} \widehat{a} e^{\frac{\mathcal{E} \mathcal{D} \phi T}{1+\mathcal{D} \phi T}}
$$

and for molecular diffusion to be of the same order with the rate of reactant consumption, the boundarylayer thickness, say $\delta$, should be $\delta=\mathcal{O}\left(\mathcal{D}^{-1 / 2}\right)$. Let us assume that in the boundary layer the Arrhenius term varies slowly and as a first approximation it can be treated as a constant, an assumption which allows us to make analytical progress. For convenience we let $\Pi^{2}$ denote the value of this constant. The validity of this assumption will be evaluated on an a posteriori basis once the temperature distribution is known.

Hence, in the boundary layer the mass transport equation is given by

$$
0=\widehat{a}_{Y Y}-\widehat{a} \Pi^{2}
$$

which has the general solution:

$$
\widehat{a}=z_{0} e^{\Pi Y}+z_{1} e^{-\Pi Y} .
$$

This inner solution must match asymptotically the outer solution, namely,

$$
\lim _{y \rightarrow 1} \widehat{a}_{\text {outer }}=\lim _{Y \rightarrow \infty} \widehat{a}_{\text {inner }} .
$$

Now $\widehat{a}_{\text {outer }}=0$, thus we require $z_{0}=0$ for the solutions to match. Hence,

$$
\widehat{a}=z_{1} e^{-\Pi Y} .
$$

The inner solution must satisfy $a=0$ on the surface, i.e. $\widehat{a}=1$ on $Y=0$, thus $z_{1}=1$ which yields,

$$
\widehat{a}=e^{-\Pi Y} .
$$

As the outer solution is a constant, the inner solution is a uniform approximation valid everywhere. Thus, returning to the original $y$ coordinate and concentration $a$, the uniform approximation is $a=a_{F}^{f}$ with

$$
a_{F}^{f}=\mathcal{D}^{-1}\left(e^{\Pi \mathcal{D}^{1 / 2}(y-1)}-1\right),
$$

where the index $f$ denotes 'fast reaction'. The solution in (3.3a) reveals the presence of the boundary layer for the concentration: the first term in the brackets is exponentially small for $y<1$ so that the concentration is everywhere $-1 / \mathcal{D}$ except close to the surface for $y-1=\mathcal{O}\left(\mathcal{D}^{-1 / 2}\right)$ where the two terms in the brackets are of the same order and where the concentration varies rapidly to approach zero.

We now turn to the temperature field. Summing up the flat film concentration and energy equations gives, $T_{y y}+a_{y y}=0$, and hence as $a$ and therefore $a_{y y}$ vary fast in the surface boundary layer, at least $T_{y y}$ has a boundary layer at the surface. We shall demonstrate that $T_{y}$ also has a boundary layer at the surface, but not $T$. Indeed, the solution for $T$ satisfies all boundary conditions without the need to introduce a boundary layer.

Consider the energy transport equation:

$$
0=T_{y y}+e^{\Pi \mathcal{D}^{1 / 2}(y-1)} e^{\frac{\mathcal{E} \mathcal{D} \phi T}{1+\mathcal{D} \phi T}} .
$$

Outside the boundary layer the term $e^{\Pi \mathcal{D}^{1 / 2}(y-1)}(\equiv 1+\mathcal{D} a)$ is exponentially small and since the Arrhenius term has an upper bound of $e^{\mathcal{E}}(\mathcal{E} \mathcal{D} \phi T /(1+\mathcal{D} \phi T)<\mathcal{E})$, the product of the two terms is still exponentially 
small. Hence, the actual value of the Arrhenius term outside the boundary layer is irrelevant. At the same time, inside the boundary layer we have assumed that the Arrhenius term is approximately a constant, $\Pi^{2}$. Thus, for simplicity we shall set the Arrhenius term identical to $\Pi^{2}$ throughout the domain. The energy equation then reduces to

$$
0=T_{y y}+\Pi^{2} e^{\Pi \mathcal{D}^{1 / 2}(y-1)}
$$

with solution,

$$
T=-\mathcal{D}^{-1} e^{\Pi \mathcal{D}^{1 / 2}(y-1)}+z_{2} y+z_{3}
$$

We require that $T=0$ on $y=0$ and asymptotically we have $z_{3}=0$. Thus the temperature is given by:

$$
T=z_{2} y-\mathcal{D}^{-1} e^{\Pi \mathcal{D}^{1 / 2}(y-1)} .
$$

Now $T_{y}+B T=0$ on $y=1$, hence we obtain $z_{2}=\mathcal{D}^{-1}\left(\Pi \mathcal{D}^{1 / 2}+B\right) /(1+B)$ and thus the temperature is given by $T=T_{F}^{f}$ where,

$$
T_{F}^{f}=\frac{1}{\mathcal{D}}\left[\frac{\Pi \mathcal{D}^{1 / 2}+B}{1+B} y-e^{\Pi \mathcal{D}^{1 / 2}(y-1)}\right] .
$$

In the boundary layer where $y-1=\mathcal{O}\left(\mathcal{D}^{-1 / 2}\right), T \sim \mathcal{D}^{-1}(1+B)^{-1}\left(\Pi \mathcal{D}^{1 / 2}+B\right) \sim \mathcal{D}^{-1 / 2}$ and thus $\mathcal{D} T=\mathcal{O}\left(\mathcal{D}^{1 / 2}\right)$ there which $\rightarrow \infty$ as $\mathcal{D} \rightarrow \infty$, i.e. $T$ does not tend to zero in the boundary layer faster than $1 / \mathcal{D}$ as $\mathcal{D} \rightarrow \infty$. Therefore, as assumed earlier, in the boundary layer the Arrhenius term $e^{\frac{\mathcal{E} \mathcal{D} \phi T}{1+\mathcal{D} \phi T}}$ can be approximated by $e^{\mathcal{E}}$. Thus, we have determined the value of $\Pi$ :

$$
\Pi=e^{\mathcal{E} / 2} .
$$

The solution in (3.3b) reveals the presence of a boundary layer for $T_{y}$ and $T_{y y}$ but not for $T$. Indeed, for $T$ the first term in the brackets which is of $\mathcal{O}\left(\mathcal{D}^{1 / 2}\right)$ dominates over the second term which is exponentially small for $y<1$ and unity for $y=1$. For $T_{y}$, however, both terms are of the same order for $y-1=\mathcal{O}\left(\mathcal{D}^{-1 / 2}\right)$, indicating the presence of a boundary layer close to the surface for $T_{y}$. For $T_{y y}$ we loose the first term so that $T_{y y}$ vanishes everywhere except for $y-1=\mathcal{O}\left(\mathcal{D}^{-1 / 2}\right)$ where it is $\sim-\Pi^{2}$.

Apart from obtaining the concentration and energy profiles for large $\mathcal{D}$, an important result of the boundary-layer analysis presented here is that even though the Arrhenius term varies in [0,1] (e.g. it takes on the values 1 at $y=0$ and $\sim e^{\mathcal{E}}$ at $y \sim 1$ ) but is approximately a constant in the boundary layer, it can be treated as a constant throughout. This substantially simplifies the analysis of the linear/nonlinear regimes for the large $\mathcal{D}$ case to be presented in subsequent sections.

In figure 2 the analytical solutions for both small $\mathcal{D}$ and large $\mathcal{D}$ are compared with the numerical solution to system (3.1) and are found to be in excellent agreement.

\section{Linear stability analysis}

\subsection{The Orr-Sommerfeld eigenvalue problem}

We now examine the linear stability of the flat-film solution which can be written as

$$
\bar{h}=1, \quad \bar{v}=0, \quad \bar{u}=y-\frac{1}{2} y^{2}, \quad \bar{p}=(1-y) \cot \beta, \quad \bar{a}=f(y), \quad \bar{T}=g(y)
$$

where

$$
f_{y y}=(1+\mathcal{D} f) e^{\frac{\mathcal{E} \mathcal{D} \phi g}{1+\mathcal{D} \phi g}}, \quad g_{y y}=-(1+\mathcal{D} f) e^{\frac{\mathcal{E} \mathcal{D} \phi g}{1+\mathcal{D} \phi g}}
$$


subject to the boundary conditions:

$$
f_{y}(0)=f(1)=0, \quad g(0)=g_{y}(1)+B g(1)=0 .
$$

Small disturbances to the flat film solution can be examined by introducing

$$
\begin{gathered}
h=\bar{h}+\chi \tilde{h}(x, t), u=\bar{u}(y)+\chi \tilde{u}(x, y, t), v=\bar{v}(y)+\chi \tilde{v}(x, y, t) \\
p=\bar{p}(y)+\chi \tilde{p}(x, y, t), a=f(y)+\chi \tilde{a}(x, y, t), \quad T=g(y)+\chi \tilde{T}(x, y, t)
\end{gathered}
$$

where $\chi$ is a small parameter. Substituting these expressions into (2.10) and taking the limit $\chi \rightarrow 0$, yields the following linearized problem,

$$
\begin{gathered}
\tilde{u}_{x}+\tilde{v}_{y}=0 \\
\tilde{u}_{y y}=\tilde{p}_{x}+3 \operatorname{Re}\left(\tilde{u}_{t}+\bar{u} \tilde{u}_{x}+\tilde{v} \bar{u}_{y}\right)-\tilde{u}_{x x} \\
\tilde{v}_{y y}=\tilde{p}_{y}+3 \operatorname{Re}\left(\tilde{v}_{t}+\bar{u} \tilde{v}_{x}\right)-\tilde{v}_{x x} \\
\tilde{a}_{y y}-\frac{\mathcal{D} f_{y y} \tilde{a}}{1+\mathcal{D} f}-\frac{\mathcal{E} \mathcal{D} \phi f_{y y} \tilde{T}}{(1+\mathcal{D} \phi g)^{2}}=3 \operatorname{ReSc}\left(\tilde{a}_{t}+\bar{u} \tilde{a}_{x}+\tilde{v} f_{y}\right)-\tilde{a}_{x x} \\
\tilde{T}_{y y}-\frac{\mathcal{D} g_{y y} \tilde{a}}{1+\mathcal{D} f}-\frac{\mathcal{E D} \phi g_{y y} \tilde{T}}{(1+\mathcal{D} \phi g)^{2}}=3 \operatorname{Re} \operatorname{Pr}\left(\tilde{T}_{t}+\bar{u} \tilde{T}_{x}+\tilde{v} g_{y}\right)-\tilde{T}_{x x},
\end{gathered}
$$

subject to the boundary conditions at $y=0$,

$$
\tilde{u}=\tilde{v}=\tilde{a}_{y}=\tilde{T}=0
$$

and the boundary conditions at $y=1$,

$$
\begin{gathered}
\tilde{h}_{t}+\frac{1}{2} \tilde{h}_{x}=\tilde{v}, \quad \tilde{p}=\tilde{h} \cot \beta+2 \tilde{v}_{y}-(\mathrm{We}-\mathcal{M} g) \tilde{h}_{x x} \\
\tilde{a}+\tilde{h} f_{y}=0, \quad \tilde{T}_{y}+\tilde{h} g_{y y}=-B\left(\tilde{T}+\tilde{h} g_{y}\right), \quad \tilde{u}_{y}=\tilde{h}-\tilde{v}_{x}-\mathcal{M}\left(\tilde{T}_{x}+\tilde{h}_{x} g_{y}\right) .
\end{gathered}
$$

A Taylor expansion was required at $y=1$ since the interfacial boundary conditions (2.12) are evaluated at $y=h \equiv 1+\chi \tilde{h}$.

We now seek solutions of the linearized system in the form of normal modes

$$
[\tilde{h}, \tilde{u}, \tilde{v}, \tilde{p}, \tilde{a}, \tilde{T}]=e^{i k(x-c t)}\left[H, \psi_{y}(y),-i k \psi(y), \pi(y), a(y), \vartheta(y)\right],
$$

where $k$ and $c$ are the real wavenumber and complex wave velocity of the infinitesimal perturbations, respectively, with the real part $c_{r}$ the phase velocity. We can also define the complex angular frequency, $\omega=k c$, whose imaginary part, $\omega_{i}=\Im(k c)$, is the growth rate. The pressure field is then eliminated from the problem via the two momentum equations and the normal stress condition to yield the OrrSommerfeld eigenvalue problem for a falling film in the presence of an exothermic chemical reaction,

$$
\begin{gathered}
\left(D^{2}-k^{2}\right)^{2} \psi=3 i k \operatorname{Re}\left[\psi+(\bar{u}-c)\left(D^{2}-k^{2}\right) \psi\right] \\
\left(D^{2}-k^{2}\right) \mathrm{a}=\mathcal{D} f_{y y}\left(\frac{\mathrm{a}}{1+\mathcal{D} f}+\frac{\mathcal{E} \phi \vartheta}{(1+\mathcal{D} \phi g)^{2}}\right)+3 i k \operatorname{ReSc}\left[-f_{y} \psi+(\bar{u}-c) \mathrm{a}\right] \\
\left(D^{2}-k^{2}\right) \vartheta=\mathcal{D} g_{y y}\left(\frac{\mathrm{a}}{1+\mathcal{D} f}+\frac{\mathcal{E} \phi \vartheta}{(1+\mathcal{D} \phi g)^{2}}\right)+3 i k \operatorname{Re} \operatorname{Pr}\left[-g_{y} \psi+(\bar{u}-c) \vartheta\right]
\end{gathered}
$$

subject to the wall boundary conditions,

$$
\psi(0)=D \psi(0)=a_{y}(0)=\vartheta(0)=0,
$$


and the interfacial boundary conditions,

$$
\begin{gathered}
{\left[D^{2}-3 k^{2}+3 i k \operatorname{Re}\left(c-\frac{1}{2}\right)\right] D \psi(1)=i k\left[\cot \beta+k^{2}(\mathrm{We}-\mathcal{M} g)\right] H} \\
\left(D^{2}+k^{2}\right) \psi(1)=H-i k \mathcal{M}\left(\vartheta(1)+H g_{y}(1)\right) \\
a(1)+H f_{y}(1)=0 \\
D \vartheta(1)+H g_{y y}(1)=-B\left(\vartheta(1)+H g_{y}(1)\right),
\end{gathered}
$$

with

$$
H=\frac{\psi(1)}{c-1 / 2}
$$

where $D \equiv d / d y$.

\subsection{Small-wavenumber expansion}

Although a full solution to equations (4.6-4.8) can only be obtained numerically, an analytical solution is possible in the limit $k \rightarrow 0$ for the two special cases of slow and fast reaction, i.e. $\mathcal{D} \rightarrow 0$ and $\mathcal{D} \rightarrow \infty$, respectively.

\subsubsection{Slow reaction}

The solutions to $f$ and $g$ up to $\mathcal{O}(\mathcal{D})$ are given by $a$ and $T$ in Appendix B in equation $(B .1)$ with $h=1$ substituted in. Equations (4.6b) and (4.6c) can then be expanded in small $\mathcal{D}$ up to $\mathcal{O}(\mathcal{D})$ to yield:

$$
\begin{aligned}
\left(D^{2}-k^{2}\right) a & =\mathcal{D} f_{y y}(a+\mathcal{E} \phi \vartheta)+3 i k \operatorname{ReSc}\left[-f_{y} \psi+(\bar{u}-c) a\right] \\
\left(D^{2}-k^{2}\right) \vartheta & =\mathcal{D} g_{y y}(a+\mathcal{E} \phi \vartheta)+3 i k \operatorname{Re} \operatorname{Pr}\left[-g_{y} \psi+(\bar{u}-c) \vartheta\right] .
\end{aligned}
$$

By considering $k$ and $\mathcal{D}$ as two independent small parameters, we seek a solution as a double perturbation expansion in the form

$$
\Omega=\Omega_{00}+\mathcal{D} \Omega_{01}+i k\left(\Omega_{10}+\mathcal{D} \Omega_{11}\right)+\mathcal{O}\left(k^{2}, \mathcal{D}^{2}\right)
$$

where $\Omega=\{\psi, c, a, \vartheta\}$. Substituting these expansions into (4.6a), (4.9), (4.7) and (4.8) and expanding in powers of $k$ and $\mathcal{D}$ gives a sequential solution of the Orr-Sommerfeld problem for the eigenvalue $c$.

Due to the linearity and homogeneity of the equations, the leading-order solution $\psi_{00}$ is obtained within an arbitrary multiplicative constant that we set equal to unity so that $\psi_{00}$ is normalized. The algebra is rather tedious and we shall just list some of the more pertinent solutions:

$$
\begin{gathered}
\psi_{00}=y^{2}, \quad \psi_{01}=0, \quad c_{00}=1, \quad c_{01}=0, \quad \mathrm{a}_{00}=-2 \\
\vartheta_{00}=\frac{2+2 B+B^{2}}{(1+B)^{2}} y, \quad \psi_{10}=\frac{\operatorname{Re}}{20} y^{4}(y-5)+\frac{y^{3}}{3} \cot \beta, \quad \psi_{11}=0 \\
c_{10}=\frac{2}{5} \operatorname{Re}-\frac{1}{3} \cot \beta-\frac{\mathcal{M}(2+B)}{4(1+B)^{2}}, \quad c_{11}=\frac{\mathcal{M}(4+3 B)}{16(1+B)^{2}}-\frac{\mathcal{M} \phi \mathcal{E}\left(20+15 B+3 B^{2}\right)}{48(1+B)^{3}} .
\end{gathered}
$$

The above expansion yields the only root $c$ of the dispersion relation that can become unstable for moderate Reynolds numbers. There is, in fact, a countable infinite number of eigenvalues whose leadingorder terms are of the form $i c_{-1} / k$ as $k \rightarrow 0$ and which can only be destabilized for large Reynolds numbers corresponding to the Tollmien-Schlichting instability for a falling film and transition to usual turbulence.

The instability onset occurs when the coefficient of $k^{2}$ in $\omega_{i}$ vanishes, i.e. $c_{10}+\mathcal{D} c_{11}=0$, which gives the critical condition,

$$
\operatorname{Re}_{c}=\frac{5}{6} \cot \beta+\frac{5 \mathcal{M}(2+B)}{8(1+B)^{2}}-\frac{5}{2} \mathcal{D} c_{11}
$$



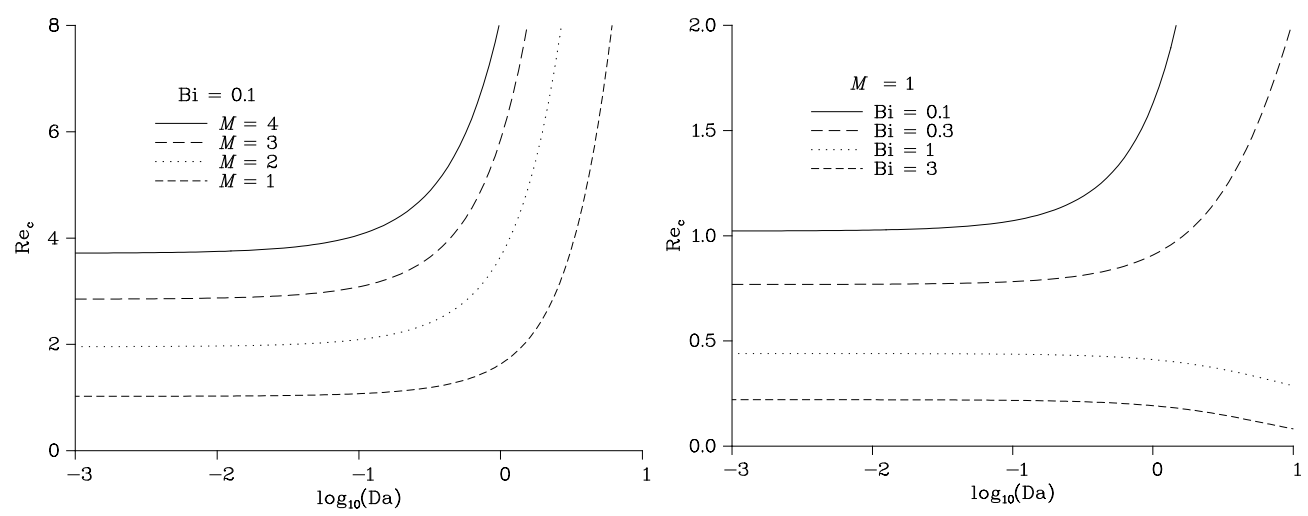

FiguRE 3. The relationship between the critical Reynolds number and Da in the slow reaction limit obtained from equation (4.10) using $\mathcal{E}=\phi=1$ and $\beta=\pi / 2$. In (a) $\mathrm{Bi}=0.1$ and $\mathcal{M}=1,2,3,4$, whilst in (b) $\mathcal{M}=1$ and $\mathrm{Bi}=0.1,0.3,1,3$.

where $\operatorname{Re}_{c}$ is the critical Reynolds number above which the flat film becomes unstable. This relationship between the critical Reynolds number and Da, in the slow reaction limit, is illustrated in figure 3, where $\mathcal{M}$ is taken to be constant.

Figures 3(a) and (b) show that the critical Reynolds number is only weakly effected by Da in the slow reaction limit (as $\bar{h}_{\mathrm{N}} \sim 1$ in the computations, the figures are qualitatively correct up to the region $\mathrm{Da} \ll 1)$. Figure $3(\mathrm{a})$ shows that increasing $\mathcal{M}$ increases the critical Reynolds number and hence the Marangoni effect induced by a slow reaction has a stabilizing influence when $\mathcal{M}>0$, (Ma $>0$ due to $\gamma>0$ ). The Reynolds number must be large enough so that inertial effects can overcome the damping effect of the reaction and lead to instability. This result can also be obtained by inspecting condition (4.10) which further reveals that the Marangoni effect is a destabilizing one for $\mathcal{M}<0$ (Ma $<0$ due to $\gamma<0)$.

Figure 3(b) reveals that increasing Bi decreases the critical Reynolds number and hence heat losses have destabilizing influence when $\mathcal{M}>0$. Again, by inspecting condition (4.10) we see that the reverse is true when $\mathcal{M}<0$. We shall return to this point in $\S 5.4$. We note that for the associated problem of thermocapillary Marangoni effect on a falling film due to heating the film uniformly from below [2124], the influence of the Marangoni effect is opposite to that in the present problem: $\gamma>0$ reduces the critical Reynolds number (by an amount proportional to the thermocapillary Marangoni number) leading to destabilization of the interface and $\gamma<0$ increases the critical Reynolds number leading to stabilization of the interface.

\subsubsection{Fast reaction}

The boundary-layer analysis of the flat film in $\S 3.2$ revealed that the Arrhenius term could be treated as a constant, $\Pi^{2}$, and equations $(4.6 \mathrm{~b})$ and $(4.6 \mathrm{c})$ reduce to

$$
\begin{aligned}
& \left(D^{2}-k^{2}\right) \mathrm{a}=\mathcal{D} \mathrm{a} \Pi^{2}+3 i k \operatorname{ReSc}\left[-f_{y} \psi+(\bar{u}-c) \mathrm{a}\right] \\
& \left(D^{2}-k^{2}\right) \vartheta=-\mathcal{D} \mathrm{a} \Pi^{2}+3 i k \operatorname{Re} \operatorname{Pr}\left[-g_{y} \psi+(\bar{u}-c) \vartheta\right],
\end{aligned}
$$

where the solutions for $f$ and $g$ are given by $a$ and $T$ in (3.3). Again we consider $k$ small and seek a solution in the form,

$$
\Omega=\Omega_{0}+i k \Omega_{1}+\mathcal{O}\left(k^{2}\right),
$$

where $\Omega=\{\psi, c, a, \vartheta\}$. Substituting these expansions into (4.6a), (4.11), (4.7) and (4.8) and expanding in powers of $k$ gives a sequential solution for the eigenvalue $c$. 

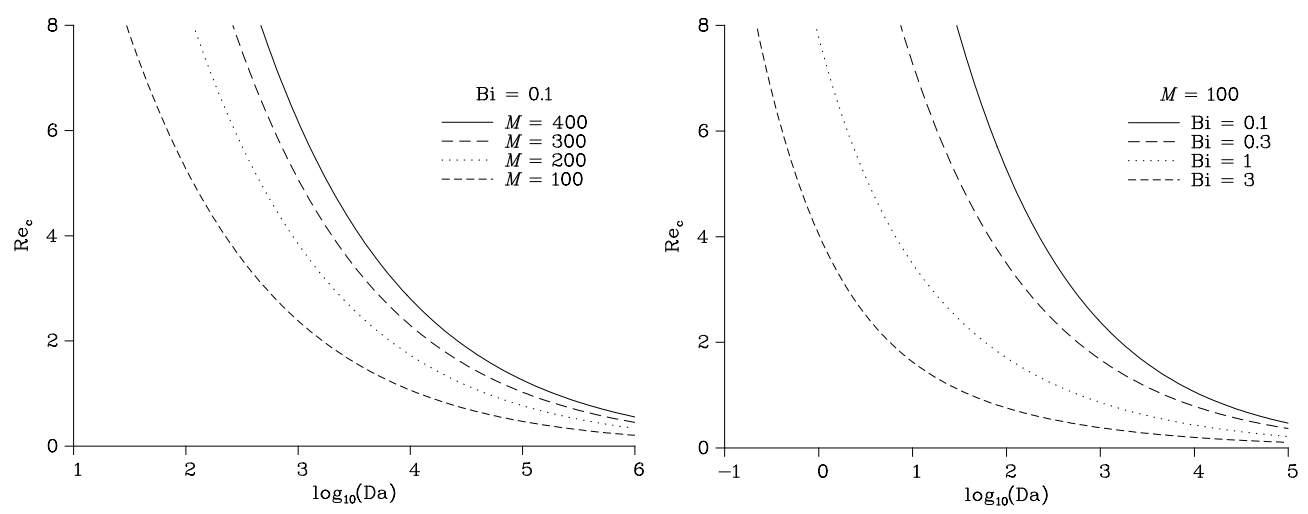

Figure 4. The relationship between the critical Reynolds number and Da in the fast reaction limit obtained from equation (4.12) using $\mathcal{E}=\phi=1$ and $\beta=\pi / 2$. In (a) $\mathrm{Bi}=0.1$ and $\mathcal{M}=100,200,300,400$, whilst in (b) $\mathcal{M}=100$ and $\mathrm{Bi}=0.1,0.3,1,3$.

The precedure for obtaining the solutions is similar to the one used in $\S 4.2 .1$ :

$$
\begin{gathered}
\psi_{0}=y^{2}, \quad c_{0}=1, \quad a_{0}=-\frac{2 \Pi}{\mathcal{D}^{1 / 2}} e^{\Pi \mathcal{D}^{1 / 2}(y-1)}, \quad \vartheta_{0}=-\mathrm{a}_{0}-\frac{2 B y\left(B+\Pi \mathcal{D}^{1 / 2}\right)}{\mathcal{D}(1+B)^{2}} \\
\psi_{1}=\frac{\operatorname{Re}}{20} y^{4}(y-5)+\frac{y^{3}}{3} \cot \beta, \quad c_{1}=\frac{2}{5} \operatorname{Re}-\frac{1}{3} \cot \beta-\frac{\mathcal{M}}{2 \mathcal{D}(1+B)^{2}}\left[B+\Pi \mathcal{D}^{1 / 2}\right] .
\end{gathered}
$$

Again, this expansion yields the only root $c$ of the dispersion relation that can become unstable.

The instability onset occurs at $c_{1}=0$ which yields the critical Reynolds number

$$
\operatorname{Re}_{c}=\frac{5}{6} \cot \beta+\frac{5 \mathcal{M}\left(B+\Pi \mathcal{D}^{1 / 2}\right)}{4 \mathcal{D}(1+B)^{2}},
$$

We note that $\mathcal{M}$ should be of $\mathcal{O}\left(\mathcal{D}^{1 / 2}\right)$ for the Marangoni effect induced by the chemical reaction to remain an $\mathcal{O}(1)$ effect. The relationship between the critical Reynolds number and Da, in the fast reaction limit, is illustrated in figure 4 .

Figures 4(a) and (b) show that the critical Reynolds number tends to zero as Da tends to infinity. Figure 4(a) shows that increasing $\mathcal{M}$ increases the critical Reynolds number and hence the Marangoni effect induced by a fast reaction also has a stabilizing influence, when $\mathcal{M}>0$. This result can also be obtained by inspecting condition (4.12) which reveals that the Marangoni effect is a destabilizing one for $\mathcal{M}<0$ ( Ma $<0$ due to $\gamma<0)$, like the slow-reaction case.

Figure 4(b) shows that increasing Bi decreases the critical Reynolds number and hence heat losses have destabilizing influence when $\mathcal{M}>0$, like the slow-reaction case. Again, by inspecting condition (4.12) we see that the reverse is true when $\mathcal{M}<0$.

Interestingly, figures 3 and 4 suggest the existence of an 'optimum' stabilizing reaction rate, in the sense that there should be a maximum for $\operatorname{Re}_{c}$ as a function of $D a$, at least for sufficiently small $B i$.

Finally, it is noteworthy that for $\mathcal{M}=0$ the above expressions for $\operatorname{Re}_{c}$ in $(4.10,4.12)$ reduce to the well-known critical condition for a free falling film, $\operatorname{Re}_{c}=(5 / 6) \cot \beta[25,26]$ (the term 'free falling film' is used hereinafter to denote the classical case of a film falling down a planar substrate without any additional effects, e.g. Marangoni effects, chemical reactions etc.).

\section{Long-wave expansion}

By introducing a formal parameter $\epsilon$ representing a typical slope of the film, we can perform a gradient expansion for $\epsilon \ll 1$. This is based on the observation that because surface tension is generally large the 
interfacial waves are typically long compared to the film thickness. This so-called 'long-wave approximation' has been central to many thin film studies (see e.g. $[1,2]$ ). We note, however, that unlike the classical 'long-wave expansion' (LWE) performed initially by Benney [27], the term 'gradient expansion' adopted here and in our previous studies on film flows $[23,24,28,29]$ refers to the particular way we do the LWE: (i) $\epsilon$ is introduced from $\epsilon \sim \partial_{x}$, $\partial_{t}$, i.e. it acts as an 'ordering parameter' instead of defining it e.g. from $\bar{h}_{\mathrm{N}} / \ell$ where $\ell$ an a-priori unknown long-scale in the streamwise direction; (ii) the LWE with respect to $\epsilon$ is then carried out as usual, i.e. it is a regular perturbation expansion of all pertinent variables in terms of $\epsilon$.

We now assume that $\epsilon^{2} \mathrm{We}, \beta, \mathcal{E}, \phi$, Re and $B$ are all $\mathcal{O}(1)$. Under these orders-of-magnitude assignments we find that the leading-order pressure is given by:

$$
p_{00}=\cot \beta(h-y)-\mathrm{We}_{x x} .
$$

The remaining variables shall be obtained through a gradient expansion for the two limiting cases of small and large $\mathcal{D}$.

\subsection{Slow reaction}

For the small $\mathcal{D}$ case we assume that $\mathcal{M}$ is $\mathcal{O}(1)$. We also make two further assumptions, $\epsilon \operatorname{Sc}$ is $\mathcal{O}(\mathcal{D})$ and $\epsilon \operatorname{Pr}$ is $\mathcal{O}\left(\mathcal{D}^{2}\right)$, so that the convective effects in both heat and mass transport are included at the lowest possible order without having to continue the gradient expansion up to $\mathcal{O}\left(\epsilon^{2}\right)$. This level of truncation allows the derivation of a relatively simple long-wave evolution equation as the $\mathcal{O}\left(\epsilon^{2}\right)$ terms are rather lengthy. The governing equations are then approximated through a double perturbation expansion (a combined gradient/small- $\mathcal{D}$ expansion):

$$
\Omega=\Omega_{00}+\mathcal{D} \Omega_{01}+\mathcal{D}^{2} \Omega_{02}+\epsilon\left(\Omega_{10}+\mathcal{D} \Omega_{11}+\mathcal{D}^{2} \Omega_{12}\right)+\mathcal{O}\left(\mathcal{D}^{3}, \epsilon^{2}\right)
$$

where $\Omega=\{u, v, a, T\}$.

As the temperature only affects the fluid flow through the tangential stress condition at $\mathcal{O}(\epsilon)$, the temperature and concentration do not require their $\mathcal{O}(\epsilon)$ corrections which would then introduce $\mathcal{O}\left(\epsilon^{2}\right)$ corrections for the velocity which are rather lengthy as already pointed out above. At this level of truncation, the concentration and temperature are given by

$$
\begin{gathered}
a=a_{L}^{s}+\mathcal{O}\left(\epsilon, \mathcal{D}^{3}\right) \\
T=T_{L}^{s}+\mathcal{O}\left(\epsilon, \mathcal{D}^{3}\right),
\end{gathered}
$$

where the subscript $L$ is used to denote 'long-wave' and the functions $a_{L}^{s}$ and $T_{L}^{s}$ are given in Appendix B. The $u$-velocity is also given in Appendix B. Then the $v$-velocity can be obtained from the continuity equation in (2.10a) which by utilizing the non-slip condition can be integrated once to give, $v=-\int_{0}^{y} u_{x} d y^{\prime}$. With the introduction of the flow rate $q^{s}=\int_{0}^{h} u d y$ in the streamwise direction, the kinematic boundary condition in $(2.12 \mathrm{c})$ can then be written as

$$
h_{t}+q_{x}^{s}=0,
$$

where $q^{s}$ is given in Appendix B. This is the long-wave equation in the slow reaction case and it will be referred to hereinafter as LWE-S.

\subsection{Fast reaction}

As before, the case of large $\mathcal{D}$ requires special treatment. We shall assume $\mathcal{M} \sim \mathcal{D}^{1 / 2}$ so that the Marangoni effect affects the $\mathcal{O}(\epsilon)$ correction to the leading-order velocity field. We also consider $\epsilon \mathrm{Sc} / \mathcal{D}$ and $\epsilon \operatorname{Pr} \sim \mathcal{O}(\Delta)$ with $\Delta$ a parameter that represents the effect of convection and is such that, $0<\epsilon \ll \Delta \ll 1$, which again allows us to include the convective effects associated with heat and mass transport at the lowest possible order without having to continue the gradient expansion up to $\mathcal{O}\left(\epsilon^{2}\right)$. 
The governing equations can then be expanded in both small $\epsilon$ and $\Delta$ as a double perturbation expansion (a combined gradient/small- $\Delta$-expansion)

$$
\Omega=\Omega_{00}+\Delta \Omega_{01}+\epsilon\left(\Omega_{10}+\Delta \Omega_{11}\right)+\mathcal{O}\left(\Delta^{2}, \epsilon^{2}\right)
$$

where $\Omega=\{u, v, a, T\}$. The expansion is truncated at $\mathcal{O}(\epsilon \Delta)$ and higher-order terms are neglected. Once again, since the temperature only effects the fluid flow through the tangential stress condition at $\mathcal{O}(\epsilon)$ the temperature and concentration do not require the $\mathcal{O}(\epsilon)$ terms at the level of truncation for the above expansion. From our discussion at the start of $\S 3$ and the boundary-layer analysis in $\S 3.2$, the leading-order velocity, concentration and temperature solutions are given in Appendix C. Then the $\mathcal{O}(\Delta)$ terms $a_{0,1}$ and $T_{0,1}$, the $\mathcal{O}(\epsilon) u$-velocity term $u_{1,0}$ and the $\mathcal{O}(\epsilon \Delta) u$-velocity term $u_{1,1}$ are also given in Appendix C. We note that $u_{01} \equiv 0$ and $u_{10}$ and $u_{11}$ have no exponential terms as they are affected by the leading-order temperature gradient through the Marangoni effect at $y=h$. As with the slow reaction case, the $v$-velocity can be obtained from, $v=-\int_{0}^{y} u_{x} d y^{\prime}$, and with the introduction of the flow rate $q^{f}=\int_{0}^{h} u d y$ in the streamwise direction, the kinematic boundary condition in $(2.12 \mathrm{c})$ can then be written as

$$
h_{t}+q_{x}^{f}=0,
$$

The function $q^{f}$ is given in Appendix C. This is the long-wave equation for the fast reaction case and will be referred to hereinafter as LWE-F.

\subsection{Rescaling}

It is now convenient to rescale the space and time coordinates for both slow and fast reaction cases as $(x, t)=\mathrm{We}^{1 / 3}(X, \Theta)$ to yield,

$$
h_{\Theta}+h^{2} h_{X}+\left[\mathcal{A} h_{X}+\mathcal{B} h_{X}^{2}+\mathcal{C} h_{X X}+\frac{h^{3}}{3} h_{X X X}\right]_{X}=0
$$

so that the coefficient of the highest derivative is independent of the parameters. The functions $\mathcal{A}, \mathcal{B}$ and $\mathcal{C}$ are given in Appendix D for both the slow and fast reaction cases.

\subsection{Linear stability of trivial solution}

We now consider the stability of the trivial solution to infinitesimal disturbances in the form of the normal mode, $h=1+H \exp \{i K(X-c \Theta)\}\left(K \mathrm{We}^{-1 / 3}=k\right.$, the wavenumber in $\left.\S 4\right)$, which when substituted into the evolution equation in (5.3) and linearizing for $H \ll 1$ yields

$$
c=i K\left(\overline{\mathcal{A}}-\frac{1}{3} K^{2}\right)+\left(1-\overline{\mathcal{C}} K^{2}\right),
$$

where the functions $\mathcal{A}$ and $\mathcal{C}$ are defined in $\S 5.3$ and the overbars denote evaluation of the corresponding functions at the flat reactive film $h=1$. Hence, the normal modes of the flat film represent infinitesimal disturbances traveling downstream with a phase velocity, $\left(1-\overline{\mathcal{C}} K^{2}\right)$. The presence of the dispersive term $-\overline{\mathcal{C}} K^{2}$ in the expression for the phase velocity implies that the convective terms of the heat/mass transport equations make the reactive falling film dispersive.

The instability onset occurs when the coefficient of $K^{2}$ in the growth rate, $\omega_{i}=\Im(K c) \equiv K^{2}(\overline{\mathcal{A}}-$ $\left.(1 / 3) K^{2}\right)$, vanishes, i.e. $\overline{\mathcal{A}}=0$, which yields the critical Reynolds number,

$$
\operatorname{Re}_{c}=\frac{5}{6} \cot \beta+\frac{5}{2} \mathcal{M} \bar{G}
$$

where $\bar{G}$ denotes $G^{s}$ (after neglecting terms of $\mathcal{O}\left(\mathcal{D}^{2}\right)$ and higher) or $G^{f}$ evaluated at $h=1$ (see Appendix D):

$$
\bar{G}^{s}=\frac{2+B}{4(1+B)^{2}}-\frac{\mathcal{D}}{16}\left(\frac{4+3 B}{(1+B)^{2}}-\mathcal{E} \phi \frac{20+15 B+3 B^{2}}{3(1+B)^{3}}\right), \quad \bar{G}^{f}=\frac{\Pi \mathcal{D}^{1 / 2}+B}{2 \mathcal{D}(1+B)^{2}}
$$


Hence, the critical Reynolds numbers predicted by LWE for both the small- and large- $\mathcal{D}$ limits are identical to equations $(4.10,4.12)$ obtained from the Orr-Sommerfeld analysis. This is not surprising as LWE is a regular perturbation expansion of the full Navier-Stokes/energy equations and associated wall and free-surface boundary conditions and hence it should resolve fully the linear instability threshold. [As far as critical/interfacial quantities are concerned to be precise; clearly due to the underlying longwave assumption, LWE fails to describe accurately the dynamics within the film, e.g. the development of short-scale convection cells due to the Marangoni effect (as defined in [48]); a nice discussion of the relation between long- and short-wave Marangoni instability is given in [49].] We note that the expression $\omega_{i}=\left(\overline{\mathcal{A}}-(1 / 3) K^{2}\right) K^{2}$ indicates that the band of unstable modes shrinks when $\mathcal{M}$ increases (for Ma $\left.>0\right)$, a signature again of the stabilizing effect of the chemical reaction.

The expressions for the critical Reynolds numbers contain implicitly Re in their right-hand sides through the dependence of $\mathcal{D}$ and $B$ on $\bar{h}_{\mathrm{N}}-$ recall the relations $\operatorname{Re}=\bar{h}_{\mathrm{N}}^{3} / 3, \mathcal{D}=\operatorname{Da} \bar{h}_{\mathrm{N}}^{2}$ and $B=\operatorname{Bi} \bar{h}_{\mathrm{N}}$. By assuming $\bar{h}_{\mathrm{N}}=\mathcal{O}(1)$ (the Nusselt film thickness is of the same order with the viscous-gravity lengthscale), small $\mathcal{D}$ is equivalent to small Da. In order to maintain the Marangoni effect in this case as an $\mathcal{O}(1)$ term in the critical Reynolds number, we assume $\mathcal{M}=\operatorname{MaDa} \phi=\mathcal{O}(1)$ or equivalently $\mathrm{Ma}=\mathcal{O}(1 / \mathrm{Da})$. Writing explicitly the critical Reynolds number in (5.5) in terms of $\bar{h}_{\mathrm{N}}$,

$$
\begin{aligned}
\operatorname{Re}_{c} & =\frac{5}{6} \cot \beta+\frac{5}{2} \mathcal{M}\left[\frac{2+\mathrm{Bi} \bar{h}_{\mathrm{N}}}{4\left(1+\mathrm{Bi} \bar{h}_{\mathrm{N}}\right)^{2}}\right. \\
& \left.-\frac{\operatorname{Da} \bar{h}_{\mathrm{N}}^{2}}{16}\left(\frac{4+3 \mathrm{Bi} \bar{h}_{\mathrm{N}}}{\left(1+\mathrm{Bi} \bar{h}_{\mathrm{N}}\right)^{2}}-\mathcal{E} \phi \frac{20+15 \mathrm{Bi} \bar{h}_{\mathrm{N}}+3 \mathrm{Bi}^{2} \bar{h}_{\mathrm{N}}^{2}}{3\left(1+\mathrm{Bi} \bar{h}_{\mathrm{N}}\right)^{3}}\right)\right]
\end{aligned}
$$

which in the limit $\mathrm{Da} \rightarrow 0$ can be rearranged as a fifth-order polynomial in $\bar{h}_{\mathrm{N}}$, so that the critical Nusselt flat film thickness cannot be expressed explicitly.

On the other hand, for large $\mathcal{D}$, which is equivalent to large Da by assuming $\bar{h}_{\mathrm{N}}=\mathcal{O}(1)$, it is appropriate to assume that $\mathcal{M}=\mathcal{O}\left(\mathrm{Da}^{1 / 2}\right)$ so that the Marangoni effect can remain an $\mathcal{O}(1)$ term. Writing explicitly the critical Reynolds number in (5.5) in terms of $\bar{h}_{\mathrm{N}}$,

$$
\operatorname{Re}_{c}=\frac{5}{6} \cot \beta+\frac{5}{4} \mathcal{M} \frac{\Pi \mathrm{Da}^{1 / 2}+\mathrm{Bi}}{\mathrm{Da}_{\overline{\mathrm{N}}}\left(1+\mathrm{Bi} \bar{h}_{\mathrm{N}}\right)^{2}},
$$

which in the limit $\mathrm{Da} \rightarrow \infty$ can be rearranged as a sixth-order polynomial in $\bar{h}_{\mathrm{N}}$, so that again the critical Nusselt flat film thickness cannot be expressed explicitly.

\subsection{Mechanism}

As pointed out in $\S 4.2$, the Marangoni effect induced by an exothermic chemical reaction is stabilizing for $\gamma>0$ and destabilizing for $\gamma<0$. This can be understood by examining the fate of an initially corrugated interface in response to the Marangoni effect induced by the reaction. By utilizing the freesurface temperature distribution obtained from the long-wave approximation in Appendix B, it can be easily shown numerically that elevated regions always have higher free-surface temperatures compared to depressed regions: physically, below the peaks of the corrugation the rate of reaction is higher as there is more reactant available there (however, the free-surface concentration remains constant due to the boundary condition in (2.7)); alternatively, elevated regions are further away from the 'cold' wall and hence are hotter than depressed ones.

Consider now an elevated region with temperature $T_{1}$ and a depressed region with temperature $T_{2}<T_{1}$. Consider also four different values of $\gamma, \gamma_{A}<\gamma_{B}<0<\gamma_{C}<\gamma_{D}$. In cases A and B the surface tension gradient is directed from 2 to 1 so that an exothermic chemical reaction is destabilizing for $\gamma<0$. On the contrary, in cases $\mathrm{C}$ and $\mathrm{D}$ the surface tension gradient is directed from 1 to 2 so that an exothermic chemical reaction is stabilizing for $\gamma>0$. [As a result, for an instability the Reynolds number should not be very small so that the destabilizing inertia effect can overcome the damping effect of the chemical 
reaction.] For cases $\mathrm{C}, \mathrm{D}$ we have $\sigma_{2}^{D}-\sigma_{1}^{D}=\gamma_{D}\left(T_{1}-T_{2}\right)>\sigma_{2}^{C}-\sigma_{1}^{C}=\gamma_{C}\left(T_{1}-T_{2}\right)$ so that as $\gamma$ increases the surface tension gradient $\sigma_{2}-\sigma_{1}$ increases and the dampening effect of the chemical reaction increases leading to reduction of the perturbation. On the other hand, for cases $\mathrm{A}$ and $\mathrm{B}$ we have $\sigma_{1}^{A}-\sigma_{2}^{A}=-\gamma_{A}\left(T_{1}-T_{2}\right)>\sigma_{1}^{B}-\sigma_{2}^{B}=-\gamma_{B}\left(T_{1}-T_{2}\right)$ and the surface tension gradient increases as $|\gamma|$ increases so that the destabilizing effect of the chemical reaction increases leading to amplification of the perturbation.

Regarding the influence of the modified Biot number, clearly for $\gamma>0$ increasing $B$ reduces the stabilization induced by the Marangoni effect (e.g. in the fast reaction case increasing $B$ reduces $\bar{G}^{f}$ ). Physically, as $B$ increases more heat is transferred to the ambient gas phase which then reduces the interfacial temperature and hence its gradient so that the stabilizing effect of the chemical reaction decreases (this can be easily seen numerically by considering e.g. the flat film interfacial temperature distribution $\left.T_{F}^{s}\right|_{y=h}$ from Appendix A: this function increases monotonically when $B$ increases). The opposite is true for $\gamma<0$.

\subsection{Mass transport across the interface}

An important measure of the efficiency of the system is the mass transport across the interface. Let us consider a solution of period $L$. In dimensionless variables the total flux across the interface is given by,

$$
f_{T}=\frac{\left.\int_{0}^{L}(\nabla a \cdot \mathbf{n})\right|_{y=h} d x}{\int_{0}^{L} d x}=\left.\frac{1}{L} \int_{0}^{L}\left(a_{y}-h_{x} a_{x}\right)_{y=h} d x \approx \frac{1}{L} \int_{0}^{L} a_{y}\right|_{y=h} d x .
$$

Taking the case of a slow reaction and using the LWE solution for $a$ in Appendix B, to first order in $\mathcal{D}$ we obtain:

$$
\begin{aligned}
f_{T} & =\frac{1}{L} \int_{0}^{L}\left(h+\frac{\mathcal{D} h^{3}}{12}\left[\mathcal{E} \phi \frac{4+B h}{1+B h}-4\right]+\frac{13}{8} \operatorname{ReSch}^{4} h_{x}\right) d x \\
& \equiv \frac{1}{L} \int_{0}^{L}\left(h+\frac{\mathcal{D} h^{3}}{12}\left[\mathcal{E} \phi \frac{4+B h}{1+B h}-4\right]\right) d x
\end{aligned}
$$

We note that to leading order this quantity is the average film height and mass transport is proportional to the average film thickness. As the average film thickness being proportional to the cube root of the fluid rate when the film is flat, to a first approximation the mass transport is also approximately proportional to the cube root of the fluid rate, and increasing the flow rate increases mass transport. In dimensional quantities this becomes:

$$
F_{T}=a_{\infty}(\mathcal{D} / L) \int_{0}^{L} h d x
$$

Although it first appears that the total mass transport is proportional to the reaction rate $\mathcal{D}$, however, the integral of the film thickness will depend on $\mathcal{D}$. In fact, this integral changes dynamically and naturally, the more unstable the system, the greater this value and so the greater the mass transport rate.

For the fast reaction rate case, using the LWE solution for $a$ given in Appendix $\mathrm{C}$ we obtain:

$$
\begin{aligned}
f_{T} & =\frac{1}{L} \int_{0}^{L}\left(\frac{\Pi}{\mathcal{D}^{1 / 2}}-\frac{3 \operatorname{ReSc}}{4 \Pi \mathcal{D}^{3 / 2}} h h_{x}\right) d x \\
& =\frac{1}{L}\left[\frac{\Pi x}{\mathcal{D}^{1 / 2}}-\frac{3 \operatorname{ReSc}}{8 \Pi \mathcal{D}^{3 / 2}} h^{2}\right]_{0}^{L}=\frac{\Pi}{\mathcal{D}^{1 / 2}} .
\end{aligned}
$$

Thus in dimensional variables we have

$$
F_{T}=a_{\infty} \Pi \mathcal{D}^{1 / 2}
$$


which reveals that the mass flux across the interface is proportional to the square root of $\mathcal{D}$. Thus, the faster the reaction, the larger the mass transport across the interface. Surprisingly, this result is independent of all flow parameters implying that convection beneath a wave is not affecting the mass transport across the interface.

\section{Integral-boundary-layer approximation}

\subsection{Previous studies}

For a free falling film, [30] and [31] constructed numerically solitary waves of the first-order LWE and demonstrated that the solitary-wave solution branches for the speed of the waves as a function of the Reynolds number show branch multiplicity and turning points above which solitary waves do not exist. Time-dependent computations by Pumir et al. [30] revealed that LWE exhibits finite-time blow-up behavior when it is integrated in regions of the parameter space where solitary waves do not exist. The connection between the absence of solitary-wave solutions and finite-time blow up was investigated in detail by Oron and Gottlieb [32] and Scheid et al. [33].

Clearly, this behavior is unphysical and marks the failure of LWE to correctly describe nonlinear waves far from criticality: LWE is necessarily limited to a narrow range of parameters around the instability onset (as pointed out in $\S 5.4$ it fully resolves the linear instability threshold as far as critical/interfacial quantities are concerned). The main reason behind this failure is the slaving of all variables to the free-surface height $h$, thus yielding a single evolution equation for $h$.

A way out is to introduce more degrees of freedom/independent fields and obtain models in terms of systems of coupled evolution equations for these fields. Such systems of coupled equations can be obtained by averaging the basic equations across the fluid layer. This process enables us to turn from the description of the motion of a fluid particle to the motion of a column of fluid from $y=0$ to $y=h$. Obviously, such an approximation is valid only if a strong coherence between different layers of the fluid from $y=0$ to $y=h$ exists. As the interfacial waves are typically long compared to the film thickness, the viscosity of the fluid ensures a strong coherence of the flow across the film. The averaging process is then combined with the long-wave approximation and the resulting system of averaged equations is frequently referred to as the 'integral-boundary-layer' (IBL) approximation; for the free falling film it was introduced by Shkadov [34], following the pioneering theoretical work by Kapitza [4]. It combines the boundary-layer approximation of the Navier-Stokes equations, assuming a self-similar parabolic velocity profile and long waves on the interface, with the Kármán-Pohlhausen averaging method in boundary-layer theory. It results in a two-equation model for the free surface and streamwise flow rate and unlike LWE, IBL has no turning points for the solitary wave solution branches and in fact it predicts the existence of solitary waves for all Reynolds numbers. However, despite its success in describing nonlinear waves far from criticality, the IBL approximation does have its shortcomings the principal one being a $20 \%$ error for the critical Reynolds number (except for film flow on the vertical plane where the critical Reynolds number vanishes).

The IBL approximation was corrected by Ruyer-Quil and Manneville [35-37] who combined a gradient expansion with a weighted residual technique using polynomial test functions for the velocity field (we know that the interface can exhibit quite complex structures in space and time, however, the fluid flow beneath the surface is much simpler). Equations for the amplitudes/coefficients of each test function are then obtained by appropriate weighted averaging of the flow equations across the film. It was shown that a simple Galerkin projection with just one test function (the self-similar parabolic profile assumed by Shkadov) and a weight function the test function itself (the Kármán-Pohlhausen averaging method employed by Shkadov can be viewed as a special weighted weighted residuals technique with a weight function equal to unity), fully corrects the critical Reynolds number.

The IBL approximation for free falling films was extended to falling films in the presence of thermocapillary Marangoni effects induced by heating the substrate either uniformly or non-uniformly by Kalliadasis et al. $[21,38]$. These authors assumed a velocity profile that satisfies the free-surface boundary condition 
due to the Marangoni effect through a tau method. However, the resulting model still suffers from the main deficiency of IBL for the free falling film case, namely a $20 \%$ error for the critical Reynolds number. This deficiency was cured for the problem of a uniformly heated wall by Trevelyan and Kalliadasis [22] who combined the tau method of Kalliadasis et al. [21,38] with the simple Galerkin projection for the free falling film employed by Ruyer-Quil and Manneville (that corrects the critical Reynolds number of the IBL approach), appropriately modified for the problem of a uniformly heated wall.

The studies by Ruyer-Quil et al. [23] and Scheid et al. [28] for a falling film heated uniformly from below took into account the second-order diffusive terms of the momentum and energy equations: as was shown in the studies of Ruyer-Quil and Manneville [35-37] for the free falling film, the diffusive terms of the momentum equation play an important role in the dispersion of the capillary ripples at the front of the main solitary hump and they determine the amplitude and frequency of these ripples. The procedure Ruyer-Quil et al. [23] and Scheid et al. [28] followed is effectively an extension of the methodology employed by Ruyer-Quil and Manneville to heated falling films and is based on a high-order weighted residuals approach with polynomial expansions for both the velocity and temperature fields. The problem of a film falling down a planar substrate heated with a mixed heat flux condition that takes into account the heat losses from the substrate to the ambient gas phase was considered recently by Trevelyan et al. [24]. Finally, the more recent study by Ruyer-Quil et al. [29] for the problem of a film falling down a vertical fiber combined the gradient expansion with a weighted residuals approach and demonstrated good agreement with the experimental results by Kliakhandler et al. [39] and Duprat et al. [40].

\subsection{The momentum equation}

Here we adapt the aforementioned studies to falling films in the presence of chemical reactions. The starting point is to assume long waves in the streamwise direction, i.e. invoke the gradient expansion. We also assume that $\operatorname{Re}, \mathrm{B}=\mathcal{O}(1)$ and $\epsilon^{2} \mathrm{We}=\mathcal{O}(1)$. For the modified Marangoni number we assume $\mathcal{M}=\mathcal{O}(1)$ for the slow-reaction case. These orders-of-magnitude assignments are made for simplicity and in order to illustrate the main points of the derivation of the IBL equations. We shall return to this point shortly. For simplicity the governing equations are truncated at first order so that second and higher-order terms are neglected (i.e. we neglect the second-order diffusive terms $u_{x x}, a_{x x}$ and $T_{x x}$ ).

First we consider the fluid flow. The bulk equations (2.10) become,

$$
u_{x}+v_{y}=0, u_{y y}+1=p_{x}+3 \operatorname{Re}\left(u_{t}+u u_{x}+v u_{y}\right), \quad p_{y}=-\cot \beta
$$

subject to the wall conditions (2.11),

$$
u=0 \text { and } \quad v=0 \text { on } y=0
$$

and interfacial conditions (2.12),

$$
p+\mathrm{We} h_{x x}=0, \quad u_{y}+\mathcal{M}\left(T_{x}+h_{x} T_{y}\right)=0 \quad \text { and } \quad h_{t}+u h_{x}-v=0 \quad \text { on } \quad y=h .
$$

From (6.1a) and (6.1c) the pressure distribution is readily obtained,

$$
p=(h-y) \cot \beta-\mathrm{We}_{x x} .
$$

Substituting the expression for the pressure into the streamwise component of the momentum equation in (6.1a) yields:

$$
u_{y y}+1=h_{x} \cot \beta-\mathrm{We} h_{x x x}+3 \operatorname{Re}\left(u_{t}+u u_{x}+v u_{y}\right) .
$$

Using the continuity equation in (6.1a) and the no-penetration condition on the wall (6.1b) allows for the elimination of the $y$-component of the velocity field, $v=-\int_{0}^{y} u_{x} d \tilde{y}$. By introducing $\theta$ as the interfacial temperature, the tangential stress condition in (6.1c) becomes:

$$
u_{y}(y=h)=-\mathcal{M} \theta_{x} .
$$


By introducing now the streamwise flow rate,

$$
q=\int_{0}^{h} u d y
$$

and by integrating the continuity equation in (6.1a) across the film, the kinematic boundary condition in $(6.1 \mathrm{c})$ can be written as:

$$
h_{t}+q_{x}=0
$$

In the absence of the Marangoni effect, i.e. for $\mathcal{M}=0$, equations (6.3) together with the no-slip condition on the wall $(6.1 \mathrm{~b})$ are the 'first-order boundary-layer equations' (terms of $\mathcal{O}\left(\epsilon^{2}\right)$ and higher have been neglected) for a free falling film. Although for $\mathcal{M} \neq 0$ the boundary-layer equations for the fluid flow are coupled to the mass and energy transport equations subject to their associated boundary conditions, we can still examine the flow field by assuming that the function $\theta$ is known.

We now project the velocity field onto the set of polynomial test functions,

$$
u=\sum_{i=1}^{N} a_{i}(x, t) \eta^{i}
$$

where $\eta=y / h(x, t)$ is a reduced normal coordinate and the amplitudes $a_{i}$ have to be determined. The corresponding approximation for $v$ is then obtained from $v=-\int_{0}^{y} u_{x} d y^{\prime}$. Introduction of the profile in (6.4) into (6.3a) yields the residual:

$$
R_{u}=3 \operatorname{Re}\left(u_{t}+u u_{x}+v u_{y}\right)-u_{y y}-1+h_{x} \cot \beta-\mathrm{We} h_{x x x} .
$$

The expansion in (6.4) satisfies trivially the no-slip boundary condition in (6.1b). We also require that the integral of this expansion with respect to $y$ (or the reduced variable $\eta$ ) gives the flow rate $q$ in the streamwise direction and also satisfies the interfacial boundary condition due to the Marangoni effect induced by the chemical reaction in $(6.3 \mathrm{~b})$. These give

$$
\frac{a_{1}}{2}+\frac{a_{2}}{3}=\frac{q}{h}-\sum_{i=3}^{N} \frac{a_{i}}{i+1}
$$

and

$$
a_{1}+2 a_{2}=-\mathcal{M h} \theta_{x}-\sum_{i=3}^{N} i a_{i}
$$

which can be solved for $a_{1}$ and $a_{2}$ which in turn are substituted into (6.4) to yield

$$
u=u^{(0)}+\frac{1}{2} \mathcal{M} h \theta_{x}\left(\eta-\frac{3}{2} \eta^{2}\right)+\sum_{i=2}^{N-1} a_{i+1} \phi_{i}(\eta)
$$

where

$$
u^{(0)}=3 \frac{q}{h} \phi_{1}(\eta) \equiv 3 \frac{q}{h}\left(\eta-\frac{1}{2} \eta^{2}\right)
$$

is the self-similar semi-parabolic profile for the free falling film introduced by Shkadov [34] and, for $i=2,3,4, \cdots, N-1$,

$$
\phi_{i}(\eta)=\left(\frac{i+1}{2}-\frac{3}{i+2}\right) \eta+\frac{3}{4}\left(\frac{2}{i+2}-3(i+1)\right) \eta^{2}+\eta^{i+1} .
$$

Hence, by eliminating the amplitudes $a_{1}$ and $a_{2}$ we have introduced explicitly into the problem the streamwise flow rate $q$ and we have satisfied the tangential stress boundary condition. As a consequence 
we have reduced the number of amplitudes by one, with $q$ and $a_{i}, i=3,4,5, \cdots, N$, the amplitudes for the new set of test functions. In effect the elimination of $a_{1}$ and $a_{2}$ 'homogenizes' the tangential stress boundary condition, a step equivalent to a tau method. Alternatively, this approach can be viewed as a projection of the function

$$
u^{\prime} \equiv u-\frac{1}{2} \mathcal{M} h \theta_{x}\left(\eta-\frac{3}{2} \eta^{2}\right)
$$

onto the new set of test functions $\phi_{i}(\eta) ; i=1,2,3, \cdots, N-1$,

$$
u^{\prime}=\sum_{i=1}^{N-1} a_{i}^{\prime} \phi_{i}(\eta)
$$

where

$$
a_{1}^{\prime}=\frac{3 q}{h} \quad \text { and } \quad a_{i}^{\prime}=a_{i+1} ; \quad i=2,3, \cdots, N-1
$$

so that the tangential stress boundary condition is homogenized.

In general $N$ must be sufficiently large to achieve convergence, however, it turns out that, much like the heated falling film problem [22] a simple Galerkin projection with just the test function $\phi_{1}$ is sufficient for a satisfactory description of the behavior close to criticality and in the nonlinear regime. We shall then truncate the projection in (6.9) after the first term,

$$
u=u^{(0)}+\frac{1}{2} \mathcal{M} h \theta_{x}\left(\eta-\frac{3}{2} \eta^{2}\right),
$$

which is the simplest possible velocity profile that satisfies all boundary conditions and its integral with respect to $y$ (or the reduced variable $\eta$ ) gives the streamwise flow rate $q$. Equation (6.10) coincides with the profile proposed by Kalliadasis et al. [21,38] and Trevelyan and Kalliadasis [22] for a heated falling film when $\mathcal{M}$ is replaced with the Marangoni number for the thermocapillary Marangoni effect. We also note that the profile (6.10) trivially satisfies the $x$-component of the equation of motion for zero Reynolds and modified Marangoni numbers with $q=h^{3} / 3 \equiv 1 / 3$, i.e. when the interface is flat. [Shkadov's basic assumption for the profile in $(6.8 \mathrm{~b})$ was that the parabolic velocity profile which satisfies the $x$-component of the equation of motion for zero Reynolds number, persists even for larger Reynolds numbers when the free surface is no longer flat.]

Introducing now the profile (6.10) into the residual (6.5) and truncating the resulting expression at $\mathcal{O}(\epsilon)$ yields,

$$
\tilde{R}_{u}=3 \operatorname{Re}\left(u_{t}^{(0)}+u^{(0)} u_{x}^{(0)}+v^{(0)} u_{y}^{(0)}\right)-u_{y y}-1+h_{x} \cot \beta-\mathrm{We} h_{x x x}
$$

where $v^{(0)}=-\int_{0}^{y} u_{x}^{(0)} d y^{\prime}$. Indeed, the Marangoni terms in (6.10) are of $\mathcal{O}(\epsilon)$ and can only contribute to the viscous diffusion term $\partial^{2} / \partial y^{2}$.

The momentum residual in (6.11) is then made small by demanding it to be orthogonal to a weight function $w_{1}$ which provides a constraint on $q$ and thus a closure for the system,

$$
\left\langle\tilde{R}_{u}, w_{1}\right\rangle=0,
$$

where the inner product is defined as $\langle f, g\rangle=\int_{0}^{1} f g d \eta$ for any two functions $f$ and $g$ in $[0,1]$ with appropriate boundary conditions. Specifying the weight function fixes the particular weighted residual method being used. In the case of the Galerkin method, $w_{1} \equiv \phi_{1}$, which after integrations by parts, utilizing the Leibniz rule, the continuity equation in (6.1a), the no-slip boundary condition in (6.1b) and the kinematic boundary condition in (6.1c) yields:

$$
\frac{18}{5} \operatorname{Re}\left(q_{t}+\frac{17}{7} \frac{q}{h} q_{x}-\frac{9}{7} \frac{q^{2}}{h^{2}} h_{x}\right)+\frac{3 q}{h^{2}}=h+\mathrm{We} h h_{x x x}-h h_{x} \cot \beta-\frac{3}{2} \mathcal{M} \theta_{x} .
$$


This is the averaged momentum equation used in the remainder of the study.

Finally, we note that as mentioned earlier, the orders-of-magnitude assignments for the different dimensionless groups in the above derivation of the averaged equations were made for simplicity and in order to fix ideas. Indeed, strict orders-of-magnitude assignments for the different groups are not required and in fact the above assignments can be relaxed. For example, let us relax the order-of-magnitude assignment for Re while keeping for the remaining groups the assignments given above. $\epsilon$ Re must be at most of $\mathcal{O}(1)$, the maximum order in the right-hand-side of the streamwise momentum equation (2.10b), which automatically ensures that $\epsilon^{2} \mathrm{Re} \ll 1$ for cross-stream inertia to be negligible in the $y$-component of the momentum equation (2.10c) and Re $\ll$ We for cross-stream inertia to be negligible compared to surface tension in the pressure distribution (6.2). At the same time, for the streamwise inertia terms of $(2.10 \mathrm{~b})$ to dominate over the neglected $\mathcal{O}\left(\epsilon^{2}\right)$ terms of the right-hand-side of this equation, $\operatorname{Re} \gg \epsilon$.

As far as the modified Marangoni number is concerned, the most general order-of-magnitude assignment is, $\epsilon^{2} \ll \epsilon \mathcal{M} \ll 1$. The normal stress balance then (6.1c) remains unaltered as terms of $\mathcal{O}(\epsilon \mathcal{M})$ have been retained compared to the neglected $\mathcal{O}\left(\epsilon^{2}\right)$ terms. Similarly, the tangential stress balance $(6.3 \mathrm{~b})$ remains unaltered. Finally, the Marangoni terms in (6.10) are of $\mathcal{O}(\epsilon \mathcal{M})$ and can only contribute to the viscous diffusion term $\partial^{2} / \partial y^{2}$.

Before considering the heat/mass transport equations, it should be noted that the semi-parabolic velocity profile is not valid in the region in front of the solitary hump, where deviations to this profile due to inertia have been shown to be present (e.g. [35,41,42]). However, at least for moderate Reynolds numbers as is the case here, this region is only a small part of a solitary wave and so we anticipate that most of the heat/mass transport will take place in the solitary-hump region and the associated error should be small.

\subsection{The concentration and energy equations}

A similar approach is now applied for the concentration and temperature fields. For simplicity we assume $\mathrm{Re}, \mathrm{B}, \epsilon^{2} \mathrm{We}, \mathcal{M}, \mathrm{Pr}, \mathrm{Sc}=\mathcal{O}(1)$ like at the start of $\S 6.2$ for the momentum equation, but these orders-ofmagnitude assignments can be relaxed as we did at the end of $\S 6.2$. The bulk equations (2.10) become,

$$
\begin{array}{r}
a_{y y}=(1+\mathcal{D} a) e^{\frac{\mathcal{E D} \phi T}{1+\mathcal{D} \phi T}}+3 \operatorname{ReSc}\left(a_{t}+u a_{x}+v a_{y}\right) \\
T_{y y}=-(1+\mathcal{D} a) e^{\frac{\mathcal{E} \mathcal{D} \phi T}{1+\mathcal{D} \phi T}}+3 \operatorname{RePr}\left(T_{t}+u T_{x}+v T_{y}\right)
\end{array}
$$

with the wall conditions (2.11),

$$
a_{y}=T=0 \quad \text { on } \quad y=0
$$

and interfacial conditions (2.12),

$$
a=T_{y}+B T=0 \quad \text { on } \quad y=h .
$$

Just as for the velocity field, we shall use a single test function to approximate each of the concentration and temperature fields. And much like with the velocity field, the test function for each of the concentration and temperature fields is based on the flat film solution. However, for the velocity field the boundary conditions are satisfied through a tau method. This is not the case with the concentration/temperature fields where the boundary conditions in $(6.14 \mathrm{c}, \mathrm{d})$ are homogeneous.

\subsubsection{Slow reaction}

For a flat film, i.e. $\operatorname{Re}=0$ (equation $(\mathrm{A} .1)$ ) and in the absence of a chemical reaction, i.e. $\mathcal{D}=0$, the concentration and temperature profiles can be written as

$$
a^{s}=\left(1-\eta^{2}\right) \alpha, \quad T^{s}=(2+B h-(1+B h) \eta) \eta \theta
$$


which already satisfy the boundary conditions along with $\left.a^{s}\right|_{\eta=0}=\alpha$ and $\left.T^{s}\right|_{\eta=1}=\theta$. These are the test functions for the concentration and temperature fields in the slow reaction case. Introducing them into the bulk equations (6.14) and performing a Taylor expansion for small $\mathcal{D}$ yields the residuals,

$$
\begin{aligned}
\tilde{R}_{a}^{s} & =1+\mathcal{D}\left(a^{s}+\mathcal{E} \phi T^{s}\right)+\frac{1}{2} \mathcal{D}^{2} \mathcal{E} \phi T^{s}\left(2 a^{s}+(\mathcal{E}-2) \phi T^{s}\right)-a_{y y}^{s} \\
& +3 \operatorname{ReSc}\left(a_{t}^{s}+u^{(0)} a_{x}^{s}+v^{(0)} a_{y}^{s}\right) \\
\tilde{R}_{T}^{s} & =-1-\mathcal{D}\left(a^{s}+\mathcal{E} \phi T^{s}\right)-\frac{1}{2} \mathcal{D}^{2} \mathcal{E} \phi T^{s}\left(2 a^{s}+(\mathcal{E}-2) \phi T^{s}\right)-T_{y y}^{s} \\
& +3 \operatorname{Re} \operatorname{Pr}\left(T_{t}^{s}+u^{(0)} T_{x}^{s}+v^{(0)} T_{y}^{s}\right),
\end{aligned}
$$

where terms up to and including $\mathcal{O}\left(\mathcal{D}^{2}\right)$ have been retained while for the velocity field only the leadingorder terms have been retained; indeed, if we assume for simplicity the orders-of-magnitude assignments given at the beginning of $\S 6.2$ and $\S 6.3$, then the $\mathcal{O}(\epsilon)$ corrections to the velocity field would introduce $\mathcal{O}\left(\epsilon^{2}\right)$ corrections in the momentum equation (6.13) through $\theta_{x}$. By taking the weight functions equal to the test functions which corresponds to the Galerkin approach, the vanishing residuals for the concentration and energy equations are

$$
\left\langle 1-\eta^{2}, \tilde{R}_{a}\right\rangle=0, \quad \text { and } \quad\left\langle(2+B h-(1+B h) \eta) \eta, \tilde{R}_{T}\right\rangle=0,
$$

or

$$
0=\frac{6}{5} \operatorname{ReSc}\left[\alpha_{t}+\frac{81 q}{112 h} \alpha_{x}-\frac{31}{224 h} q_{x} \alpha\right]+\frac{\alpha}{h^{2}}+J_{e a}
$$

and

$$
\begin{aligned}
0 & =\frac{6}{5} \operatorname{RePr}\left[\left(1+\frac{7}{16} B h+\frac{1}{16} B^{2} h^{2}\right) \theta_{t}+\left(\frac{9}{7}+\frac{57 B h}{112}+\frac{15 B^{2} h^{2}}{224}\right) \frac{q}{h} \theta_{x}\right] \\
& +\frac{6}{5} \operatorname{RePr}\left[+\left(\frac{1}{7}-\frac{41 B h}{224}-\frac{27 B^{2} h^{2}}{448}\right) \theta \frac{q x}{h}+\left(\frac{57}{224}+\frac{15 B h}{224}\right) \frac{q}{h} B h_{x} \theta\right] \\
& +(4+B h)(1+B h) \frac{\theta}{4 h^{2}}-J_{e t}
\end{aligned}
$$

with the functions $J_{e a}$ and $J_{e t}$ given in Appendix E. The system of equations (6.13) and (6.18) will be referred to hereinafter as $\mathrm{IBL}^{s}$.

\subsubsection{Fast reaction}

In the case of a fast reaction, the concentration and temperature test functions are also based on the flat film solutions $a_{F}^{f}$ and $T_{F}^{f}$ given by equations (3.3) (or the functions $a_{0,0}$ and $T_{0,0}$ given in $\S 5.2$ ):

$$
a^{f}=\left(1-e^{\mathcal{D}^{\prime} h(\eta-1)}\right) \alpha, \quad T^{f}=\left(\frac{\mathcal{D}^{\prime} h+B h}{\mathcal{D}^{\prime} h-1} \eta-\frac{1+B h}{\mathcal{D}^{\prime} h-1} e^{\mathcal{D}^{\prime} h(\eta-1)}\right) \theta,
$$

where $\mathcal{D}^{\prime}=\Pi \sqrt{\mathcal{D}}$. For the flat film solution we have kept exponential terms which can be of $\mathcal{O}(1)$ near the free surface and small everywhere else and have neglected exponential terms which are small throughout $[0,1]$. Hence the flat film solution satisfies asymptotically the wall boundary conditions for $\mathcal{D} \gg 1$ and the same is true for the test functions in (6.19).

The Arrhenius term is approximated by a constant, namely $\Pi^{2}$ given in (3.4), as we did for the derivation of the flat film solutions. The resulting residuals are then given by:

$$
\begin{aligned}
& \tilde{R}_{a}^{f}=\left(1+\mathcal{D} a^{f}\right) \Pi^{2}-a_{y y}^{f}+3 \operatorname{ReSc}\left(a_{t}^{f}+u^{(0)} a_{x}^{f}+v^{(0)} a_{y}^{f}\right), \\
& \tilde{R}_{T}^{f}=-\left(1+\mathcal{D} a^{f}\right) \Pi^{2}-T_{y y}^{f}+3 \operatorname{RePr}\left(T_{t}^{f}+u^{(0)} T_{x}^{f}+v^{(0)} T_{y}^{f}\right) .
\end{aligned}
$$


Using the Galerkin approach, i.e. applying the test functions as weight functions to minimize these residuals, yields,

$$
\begin{aligned}
0 & =\operatorname{ReSc}\left[\left(1-\frac{3}{2 \mathcal{D}^{\prime} h}\right) \alpha_{t}+\left(1-\frac{9}{4 \mathcal{D}^{\prime} h}+\frac{45}{8 \mathcal{D}^{\prime 3} h^{3}}\right) \frac{q}{h} \alpha_{x}\right] \\
& +\frac{9 \operatorname{ReSc} \alpha}{16 \mathcal{D}^{\prime} h}\left[\left(2-\frac{15}{\mathcal{D}^{\prime 2} h^{2}}\right) \frac{q h_{x}}{h^{2}}-\left(2-\frac{5}{\mathcal{D}^{\prime 2} h^{2}}\right) \frac{q_{x}}{h}\right]+\frac{\Pi}{3 \mathcal{D}^{1 / 2} h}\left(\mathcal{D}^{\prime} h-1\right)(1+\mathcal{D} \alpha)
\end{aligned}
$$

and

$$
\begin{aligned}
0 & =\operatorname{Re} \operatorname{Pr}\left(\mathcal{P}_{1} \theta_{t}+9 \mathcal{P}_{2} \frac{q}{h} \theta_{x}+9 \mathcal{P}_{3} \frac{\theta q h_{x}}{h^{2}\left(\mathcal{D}^{\prime} h-1\right)}+\mathcal{P}_{4} \frac{\theta q_{x}}{8 h\left(\mathcal{D}^{\prime} h-1\right)}\right) \\
& +\frac{\theta(1+B h)}{h^{2}}\left(1-\frac{3-B h}{2 \mathcal{D}^{\prime} h}-\frac{B}{\mathcal{D}^{\prime 2} h}\right) \\
& -\frac{\alpha}{2 h}\left(1-\frac{1}{\mathcal{D}^{\prime} h}\right)^{2}\left(\mathcal{D}^{\prime 2} h+\mathcal{D}^{\prime}(B h-1)-2 B\right) \\
& -\frac{1}{2 \mathcal{D} h^{2}}\left(1-\frac{1}{\mathcal{D}^{\prime} h}\right)\left(\mathcal{D}^{\prime 2} h^{2}+\mathcal{D}^{\prime} B h^{2}-2(1+B h)\right)
\end{aligned}
$$

with the functions $\mathcal{P}_{i}$ given in Appendix E and where exponentially small terms have been neglected. The system of equations (6.13) and (6.21) will be referred to hereinafter as $\mathrm{IBL}^{f}$. The case of moderate reaction rates is treated in Appendix F.

Finally, for consistency with LWE, the $\mathrm{IBL}^{s}$ and $\mathrm{IBL}^{f}$ models are rescaled with $(x, t)=\mathrm{We}^{1 / 3}(X, \Theta)$ as we did in $\S 5.3$.

\subsection{Linear stability of trivial solution}

The trivial solution of the $\mathrm{IBL}^{s}$ model is,

$$
\begin{gathered}
h=1, \quad q=\frac{1}{3} \\
\alpha=-\frac{1}{2}+\mathcal{D}\left(\frac{1}{5}-\frac{22+7 B}{160(1+B)} \phi \mathcal{E}\right)+\mathcal{O}\left(\mathcal{D}^{2}\right) \\
\theta=\frac{1}{2(1+B)}+\frac{\mathcal{D}}{20(4+B)(1+B)}\left(-\frac{22+7 B}{2}+\frac{16+7 B+B^{2}}{1+B} \phi \mathcal{E}\right)+\mathcal{O}\left(\mathcal{D}^{2}\right)
\end{gathered}
$$

along with equation (6.15), while for the $\mathrm{IBL}^{f}$ model we have,

$$
\begin{gathered}
h=1, \quad q=\frac{1}{3} \\
\alpha=-\frac{1}{\mathcal{D}} \\
\theta=\frac{\Pi}{1+B} \frac{1}{\mathcal{D}^{1 / 2}}\left(1-\frac{1}{\Pi \mathcal{D}^{1 / 2}}\right),
\end{gathered}
$$

along with equation (6.19).

In the case of a slow reaction, only the leading-order term of the trivial solution is in agreement with the solution derived in $\S 3.1$ while for the fast reaction we obtain the same expressions with those derived in $\S 3.2$. The dispersion relation resulting from the linear stability analysis is a fourth-order polynomial for the complex wave velocity $c$. Expanding for $K \ll 1$ the only root that can become unstable yields,

$$
c=1+i \frac{1}{\mathrm{We}^{1 / 3}}\left(\frac{2}{5} \mathrm{Re}-\frac{1}{3} \cot \beta-\frac{1}{2} \mathcal{M} F\right) K+\mathcal{O}\left(K^{2}\right),
$$


where for the slow reaction $F=F^{s}$ with

$$
\begin{gathered}
F^{s}=\frac{1}{2(1+B)^{2}}\left(2+B-\frac{\mathcal{D}}{10(4+B)^{2}}\left(\frac{352+470 B+184 B^{2}+21 B^{3}}{2}\right.\right. \\
\left.\left.-\frac{256+316 B+152 B^{2}+35 B^{3}+3 B^{4}}{1+B} \phi \mathcal{E}\right)\right)
\end{gathered}
$$

and for the fast reaction, $F=F^{f}$ with:

$$
F^{f}=\frac{1}{\mathcal{D}} \frac{\Pi \mathcal{D}^{1 / 2}+B}{(1+B)^{2}} .
$$

Note that the precise orders-of-magnitude for $\mathrm{Sc}, \mathrm{Pr}$ do not matter as these parameters contribute terms of $\mathcal{O}\left(K^{3}\right)$ which are neglected here. The critical Reynolds number can then be obtained by setting the coefficient of $K^{2}$ in the growth rate, $\omega_{i}=\Im(K c)$, equal to zero,

$$
\operatorname{Re}_{c}=\frac{5}{6} \cot \beta+\frac{5}{4} \mathcal{M} F
$$

The $\mathrm{IBL}^{s}$ model gives the correct $\mathcal{O}(1)$ term for the critical Reynolds number but not the $\mathcal{O}(\mathcal{D})$ term. This is a direct consequence of not capturing correctly the $\mathcal{O}(\mathcal{D})$ terms of the flat film wall concentration and interfacial temperature. The differences are due to the fact that in order to keep the IBL models as simple as possible we used a single test function for each of the concentration and temperature. In fact, to correct the $\mathrm{IBL}^{s}$ model we would have to increase the number of test functions for both concentration and temperature (but retain the basic momentum equation in (6.13) which is responsible for the correct $\mathcal{O}(1)$ term of the critical Reynolds number).

The number of test functions depends on the level of improvement we wish to achieve. If, for example, we simply want to correct the flat film wall concentration up to and including $\mathcal{O}(\mathcal{D}$ ) (recall from $\S 3.1$ that the $\mathcal{O}\left(\mathcal{D}^{2}\right)$ terms of the concentration are not required) and the flat film interfacial temperature up to and including $\mathcal{O}\left(\mathcal{D}^{2}\right)$, the concentration and temperature must be expanded in polynomials of $\eta$ up to the powers 4 and 6 , respectively (see the distributions in Appendix B with $h_{x}=0$ ), or equivalently, 3 and 5 test functions, respectively. This would also correct $\operatorname{Re}_{c}$ up to and including $\mathcal{O}\left(\mathcal{D}^{2}\right)$. Note that $\mathrm{Re}_{c}$ is not affected by convection and indeed the flat film wall concentration and interfacial temperature do not contain any convective effects: as we pointed out in $\S 5.4$, convection affects the dispersion of the disturbances at onset but not their growth rate from which $\mathrm{Re}_{c}$ is evaluated. On the other hand, if we wish to recover from a gradient expansion LWE, the concentration and temperature must be expanded in polynomials of $\eta$ up to the powers 5 and 7, respectively, or equivalently, 4 and 6 test functions, respectively. This would fully resolve the behavior close to criticality but it would lead to a large-dimensionality IBL system.

The $\mathrm{IBL}^{f}$ model performs better than the $\mathrm{IBL}^{s}$ one as it yields the correct critical Reynolds number. However, it also does not reproduce LWE with a gradient expansion. Indeed, assuming the same ordersof-magnitude assignments for the different parameters as in the derivation of LWE, a gradient expansion of $\mathrm{IBL}^{f}$ yields to leading order

$$
\alpha_{0,0}=\frac{1}{\mathcal{D}} \quad \text { and } \quad \theta_{0,0}=\frac{\Pi \mathcal{D}^{1 / 2} h-1}{\mathcal{D}(1+B h)}
$$

from which $a_{0,0}$ and $T_{0,0}$ can be obtained through the corresponding test functions for the fast reaction case. $a_{0,0}$ and $T_{0,0}$ are then identical to the $a_{0,0}$ and $T_{0,0}$ leading-order LWE distributions given in $\S$ 4.2.2. However, the terms $a_{0,1}$ and $T_{0,1}$ which contain the heat and mass transport convective effects (through the presence of RePr and ReSc) are not the same as those given in $\S 4.2 .2$ and like with $\mathrm{IBL}^{s}$ more test 

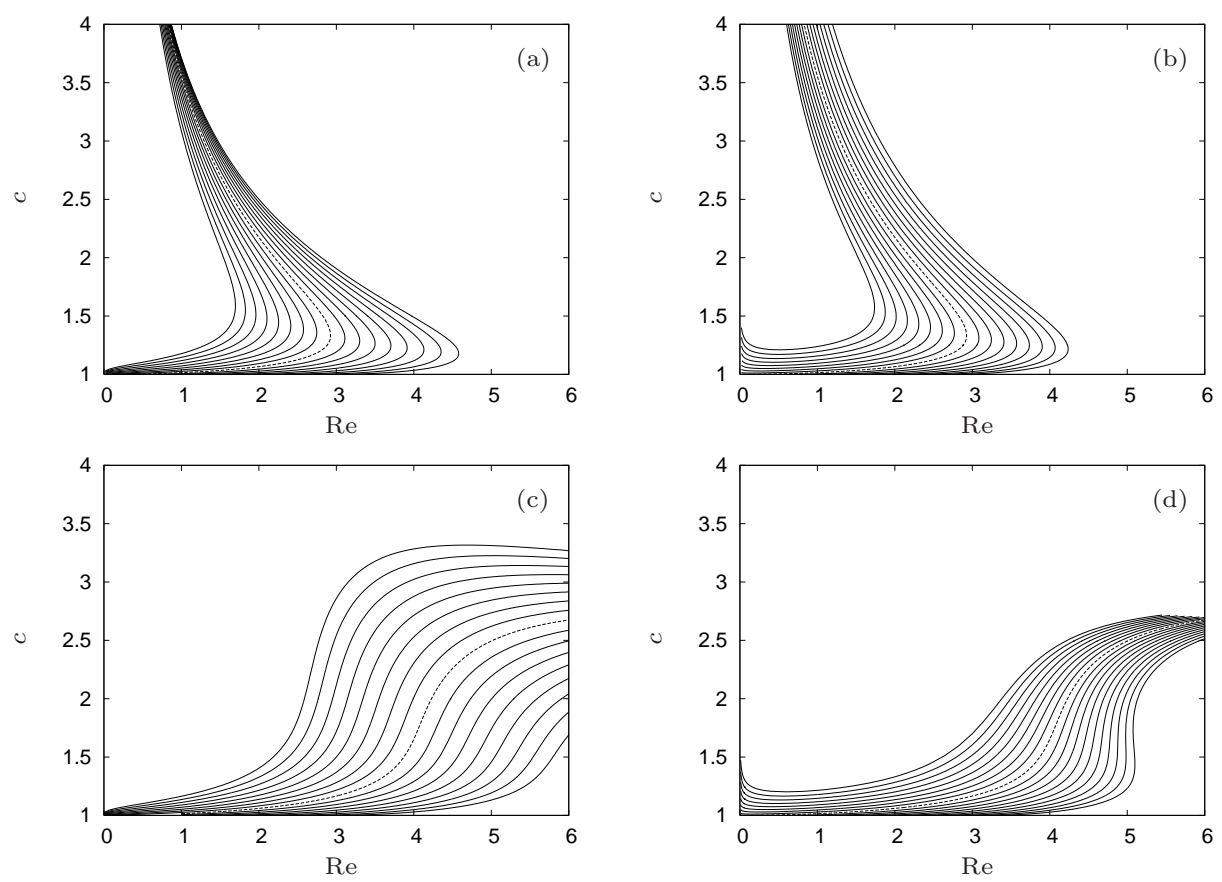

FiguRE 5. Bifurcation diagrams for the solitary waves, showing the dependence of the nonlinear eigenvalue $c$ on Re. The values of the dimensionless groups are $\Gamma=3000$, $\mathrm{Bi}=0.1, \mathcal{E}=1, \phi=1$ and $\beta=\pi / 2$. Two different values of Da are used: (i) $\mathrm{Da}=0.01$ with $\operatorname{Pr}=0.01$ and $\mathrm{Sc}=1$; (ii) $\mathrm{Da}=10000$ with $\mathrm{Pr}=1$ and $\mathrm{Sc}=10$. The dotted line corresponds to $\mathcal{M}=0$ - (a) LWE model for $\mathrm{Da}=0.01, \mathcal{M}$ changes from -3.2 to 3.2 from left to right. - (b) LWE model for $\mathrm{Da}=10000, \mathcal{M}$ changes from -400 to 400 from left to right. - (c) IBL model for $\mathrm{Da}=0.01, \mathcal{M}$ changes from -3.2 to 3.2 from left to right. - (d) IBL model for $\mathrm{Da}=10000, \mathcal{M}$ changes from -400 to 500 from left to right.

functions would be required. These observations also imply that in the artificial case $\mathrm{Sc}=\operatorname{Pr}=0$, a gradient expansion of $\mathrm{IBL}^{f}$ will give LWE.

Finally, we note that we have confirmed that with a sufficiently large number of test functions for the concentration and temperature, we do obtain IBL models whose gradient expansion close to criticality yields LWE for both slow- and fast- reaction cases. Despite the fact that the $\mathrm{IBL}^{s, f}$ models do not capture fully the instability threshold, their simplicity makes them useful prototypes for numerical exploration.

\section{Solitary waves bifurcation diagrams}

In this section we investigate the solitary waves solutions of the different models presented in the previous sections. Such waves are known to exist in the case of free falling films.

Solitary waves are obtained by seeking solutions in the form, $h(x, t)=\hat{h}(z), \theta(x, t)=\hat{\theta}(z)$, and $\alpha(x, t)=\hat{\alpha}(z)$, where $z=x-c t$ is the coordinate in a frame moving with the speed $c$ of the waves, assumed to be constant. The partial differential equations of the different models are thus brought into a set of ordinary differential equations involving $c$ as a (nonlinear) eigenvalue. Note that the resulting equation for $h$ can be integrated once as it is a conservation equation. Only solutions that tend to the same (finite) value as $z \rightarrow \pm \infty$ are retained. Such solutions correspond to homoclinic orbits in the phase plane associated with the corresponding ordinary differential system of equations. Their bifurcation diagrams are constructed numerically by using the continuation software AUTO97 by Doedel et al. [43]. 
Bifurcation diagrams for the solitary waves are depicted in figure 5. For a given Re, the wave speed decreases as $\mathcal{M}$ increases. This is an indication of the stabilizing influence of the exothermic chemical reaction on the free surface dynamics and is consistent with our observations in previous sections. A comparison between the curves obtained with the LWE models (figures 5a and 5b) and the IBL ones (figures $5 \mathrm{c}$ and $5 \mathrm{~d}$ ) confirms that, as for free falling films, the IBL models predict the continuing existence of solitary waves for all Reynolds numbers unlike the LWE models which exhibit turning points in the bifurcation diagrams. Such points are unphysical and by analogy with free falling films (recall our discussion from $\S 6.1$ ), they should be responsible for finite-time blow up behavior of the LWE models in the region where solitary waves do not exist. Note also that in figure $5 \mathrm{~d}$, two opposite turning points appear for $\mathcal{M}=450$ and $\mathcal{M}=500$, leading to hysteretic phenomena (see $\S 8$ ).

As the flow field predicted by IBL in the absence of any Marangoni effects has already been validated before [36], in Appendix G we validate our periodic traveling wave solutions for the concentration and temperature fields obtained from IBL. More specifically, we compare them with the solutions of the first order two-dimensional concentration and temperature transport equations. The fluid flow predicted from IBL in the absence of any Marangoni effects $(\mathcal{M}=0)$ is used as an input in these computations. The agreement is very good in both the small- and large-Da limits.

Waves' shapes for the film thickness $\hat{h}$, wall concentration $\hat{\alpha}$ and surface temperature $\hat{\theta}$ obtained with the IBL equations in the general case $(\mathcal{M} \neq 0)$ are depicted in figure 6.

Independently of the value of Da, the free-surface profiles are found to be similar to those obtained in the case of free falling films: they consist of a main hump, a sequence of undulations ahead at its front and a smooth tail at its back. Further, $\hat{\alpha}$ and $\hat{\theta}$ exhibit a similar behavior to $\hat{h}$, although concentration solitary waves are negative-hump ones. As a rule, the amplitude of all these waves increases as Re increases.

\section{Time-dependent computations}

To examine the spatio-temporal dynamics of the reactive film we solve numerically the IBL models as initial-value problems. We employ a Crank-Nicolson-type implicit scheme with the spatial derivatives approximated by central differences and with dynamic time step adjustment. We impose periodic boundary conditions over a finite domain much larger than the maximum growing wavelength predicted by linear stability. The computations are performed in the moving frame, $z=x-c t$, where $c$ does not necessarily correspond to the speed of the traveling waves computed in the previous section.

Typical spatio-temporal evolutions are shown in figures 7 and 8 using as initial conditions a Gaussian distribution in the middle of the domain and random noise, respectively, added to the flat film solution $h=1$. Figure 7 shows that for large times the system evolves into a single solitary pulse for each of the free surface, wall concentration and interfacial temperature, very close to the infinite-domain pulses calculated in $\S 7$ for each of these variables from the corresponding set of ordinary differential equations in terms of $z$ using AUTO97. The backwards traveling disturbances on the flat film free surface, wall concentration and interfacial temperature are actually an expanding radiation wave packet due to the primary flat film instability. In fact, in the laboratory frame of reference, both front and back of the wave packet travel in the downstream direction, with the front traveling faster than the back, typical of a convectively unstable system (e.g. [29], [40], [44] and [45]). A general rule for the interaction between wave packets and solitary waves, that seems to be applicable for both one-dimensional and two-dimensional solitary waves, is that if the speed of a solitary pulse is smaller than the largest speed in a wave packet, the speed of its front, the solitary wave cannot escape the packet and will be destroyed. On the other hand, if the speed of a solitary wave is larger than the speed of the front of a wave packet, the solitary wave will escape the packet unscathed [46,47]. Analysis of the convective instability of the reactive film is beyond the scope of this study.

In figure 8, for large times the system evolves into a train of coherent structures which resemble the infinite-domain solitary pulses for the free surface, wall concentration and interfacial temperature obtained in $\S 7$. The large-time non-stationary 'turbulent' wave dynamics observed in the figure is similar to that found in free falling films which in turn is similar to the spatio-temporal chaos observed with 

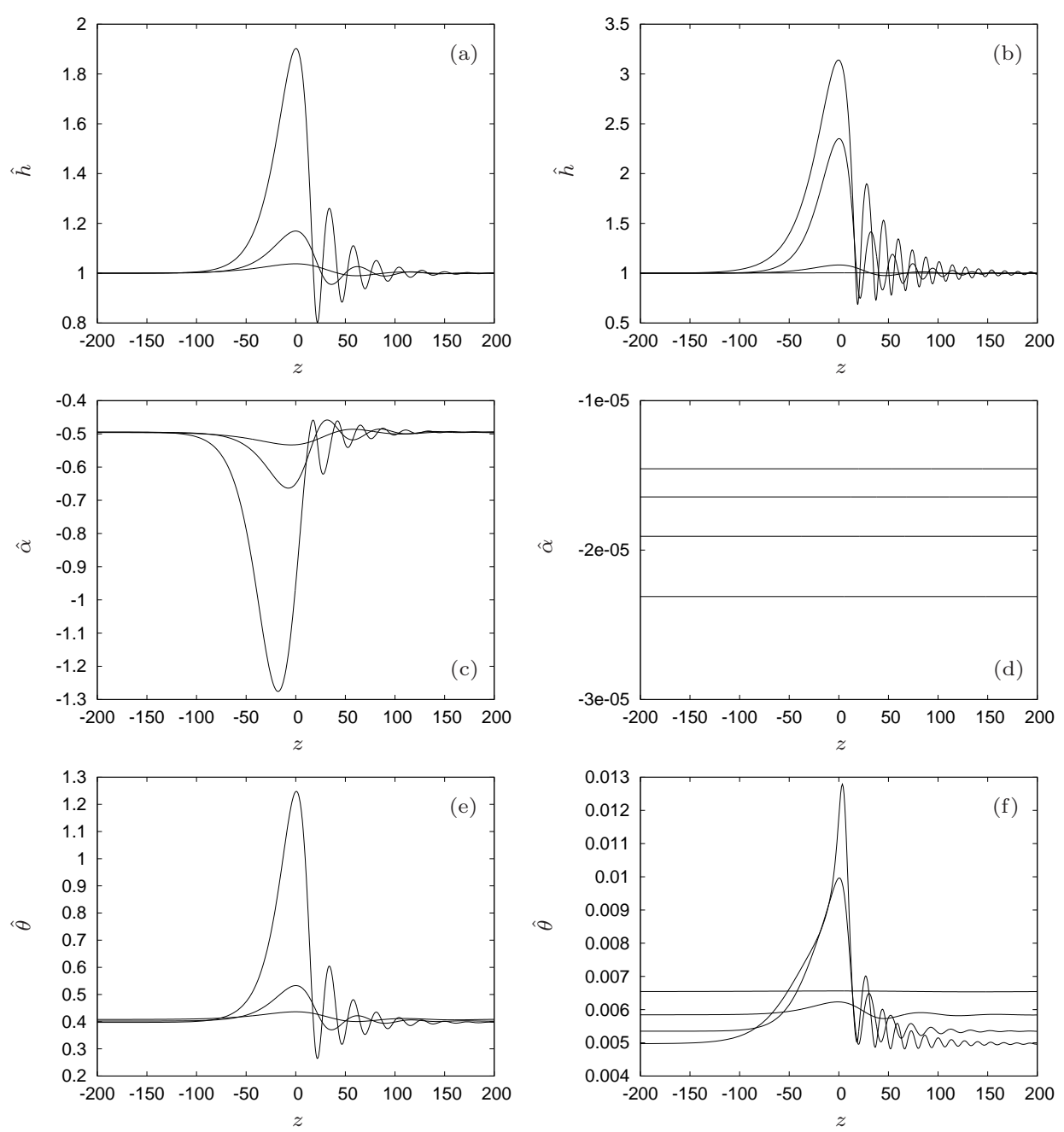

Figure 6. Profiles of the free surface thickness $\hat{h}$, wall concentration $\hat{\alpha}$ and surface temperature $\hat{\theta}$ of the traveling waves obtained with the IBL models. The values of the dimensionless groups are $\Gamma=3000, \mathrm{Bi}=0.1, \mathcal{E}=1, \phi=1$, and $\beta=\pi / 2$. (a), (c) and (e): small Da case; $\mathrm{Da}=0.01, \mathcal{M}=3.2, \mathrm{Pr}=0.01, \mathrm{Sc}=1$ and Re changes from 4 (small amplitude profiles) to 6 (large amplitude profiles) by steps of $1 .-$ (b), (d) and (f): large Da case. $\mathrm{Da}=10000, \mathcal{M}=400 \mathrm{Pr}=1, \mathrm{Sc}=10$ and Re changes from 3 (small amplitude profiles) to 6 (large amplitude profiles) by steps of 1. (In (d), Re changes from 3 to 6 from bottom to top.)

the Kuramoto-Sivashinsky and related equations. We should point out that unlike LWE which can exhibit finite-time blow up behavior in the region where solitary waves do not exist, our IBL models are quite robust and do not exhibit any singularity formation. For example, for the parameter values used in figures 7 and 8, LWE solitary waves do not exist as demonstrated in the first bifurcation diagram from the right in figure $5 \mathrm{a}$ (with the exception of Re, in figures $5 \mathrm{a}, 7$ and 8 the values for the different parameters are the same) while no blow up occurs in the IBL computations.

As noted in the previous section, for large Da and large $\mathcal{M}$, the bifurcation diagram for the IBL model is no longer monotonous but exhibits two opposite turning points, which suggests that hysteretic phenomena 


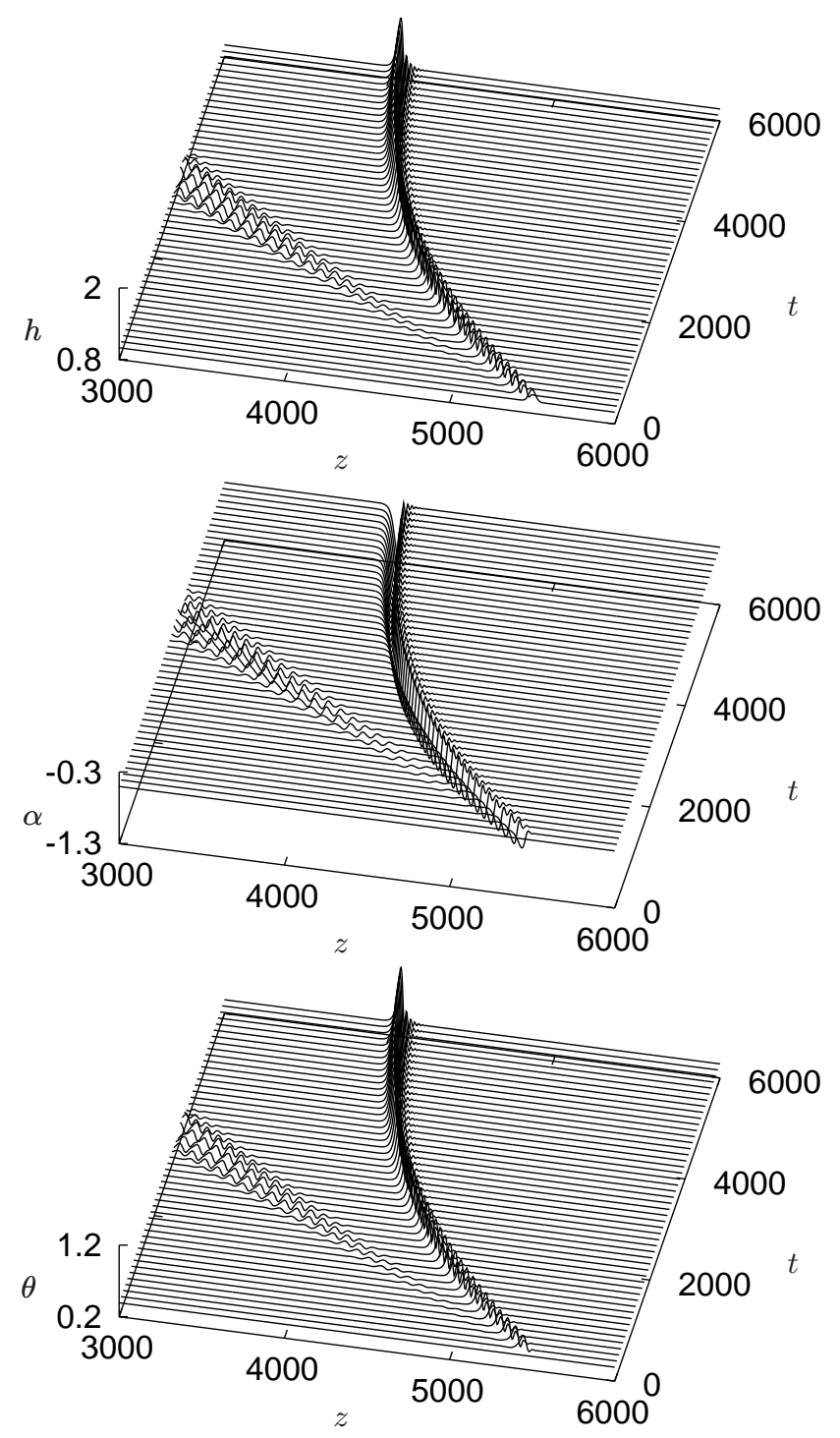

FiguRE 7. Time-evolution of the free surface $h$, wall concentration $\alpha$ and interfacial temperature $\theta$ obtained from IBL in the moving coordinate $z=x-c t$ where $c=1.68953$, the velocity of the traveling wave from figure 5 . The values of the dimensionless groups are $\Gamma=3000, \mathrm{Bi}=0.1, \mathcal{E}=1, \phi=1, \beta=\pi / 2, \mathrm{Da}=0.01, \mathcal{M}=3.2, \mathrm{Pr}=0.01, \mathrm{Sc}=1$ and $R e=6$. The initial condition is a Gaussian distribution with amplitude 0.2 at the center of the computational domain. Successive curves are separated by $\Delta t=120$.

are to be expected for that range of parameter values. This is confirmed by solving numerically the timedependent problem. In figure 9, we present two cases illustrating the evolution of a small perturbation of the film thickness. In both cases, all dimensionless groups are the same, only the amplitude of the initial perturbation is different. The value chosen for the Reynolds number is such that, according to the bifurcation diagram of figure $5 \mathrm{~d}$, three solutions co-exist. The computation reveals that, as time increases, the film surface converges indeed to different solutions depending on the amplitude of the initial perturbation, these solutions being very close both in shape and velocity to the ones obtained from 

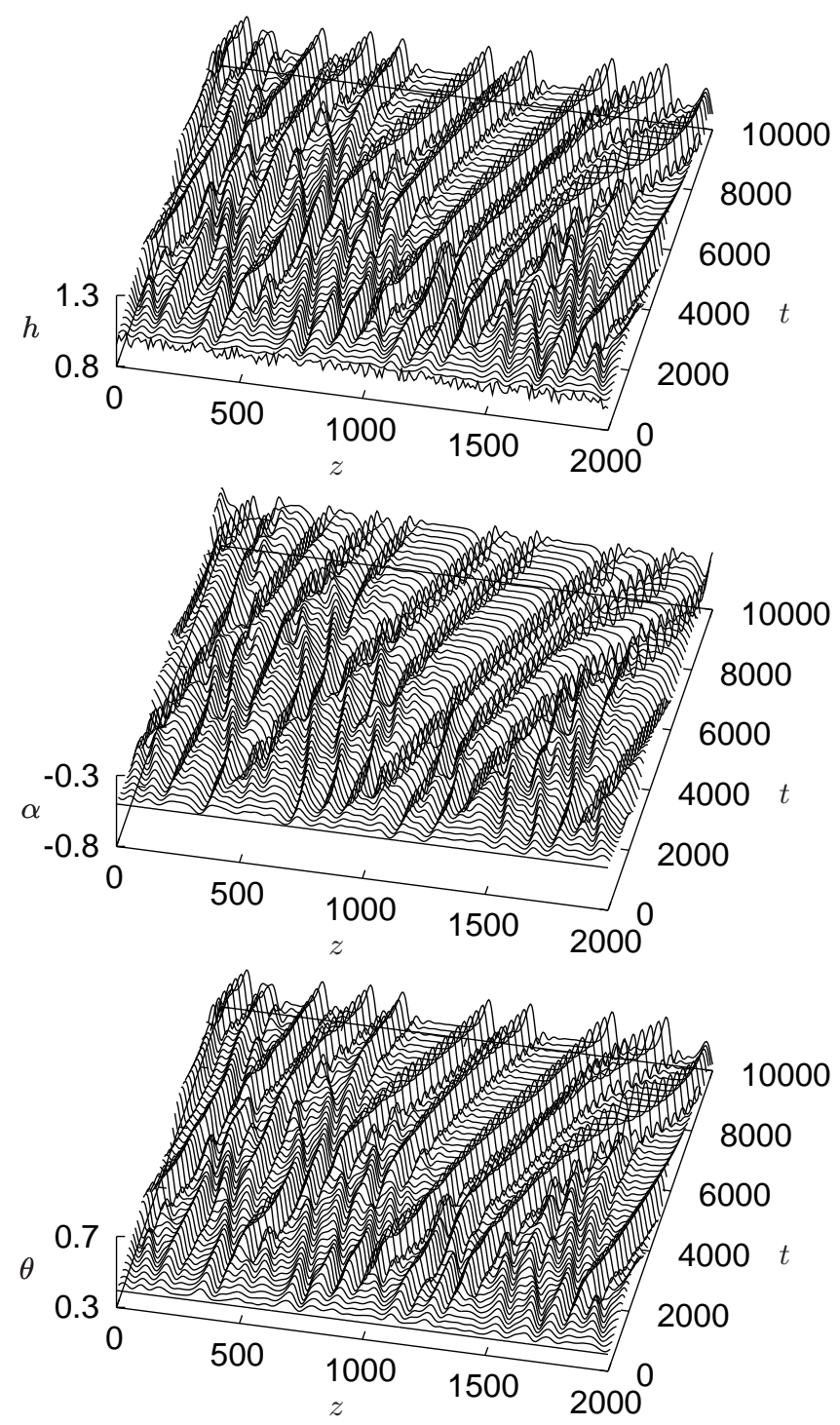

FiguRE 8. Time-evolution of the free surface $h$, wall concentration $\alpha$ and interfacial temperature $\theta$ obtained from IBL in the moving frame $z=x-c t$ where $c=1$, corresponding to the traveling wave velocity at criticality and $\mathcal{M}=0$. The values of the dimensionless groups are $\Gamma=3000, \mathrm{Bi}=0.1, \mathcal{E}=1, \phi=1, \beta=\pi / 2, \mathrm{Da}=0.01, \mathcal{M}=3.2, \operatorname{Pr}=0.01$, $\mathrm{Sc}=1$ and $\mathrm{Re}=6$. The initial condition is a random noise of maximum amplitude 0.05 . Successive curves are separated by $\Delta t=200$.

the bifurcation diagrams of $\S 7$. More precisely, the first case (small amplitude) leads to the solution of the lower branch while the second case (large amplitude) leads to the solution of the upper branch. These results are an indication of the stability of these two solutions. Regarding the solution of the middle branch, we have been unable to find a value for the initial amplitude of the perturbation leading to that solution. 

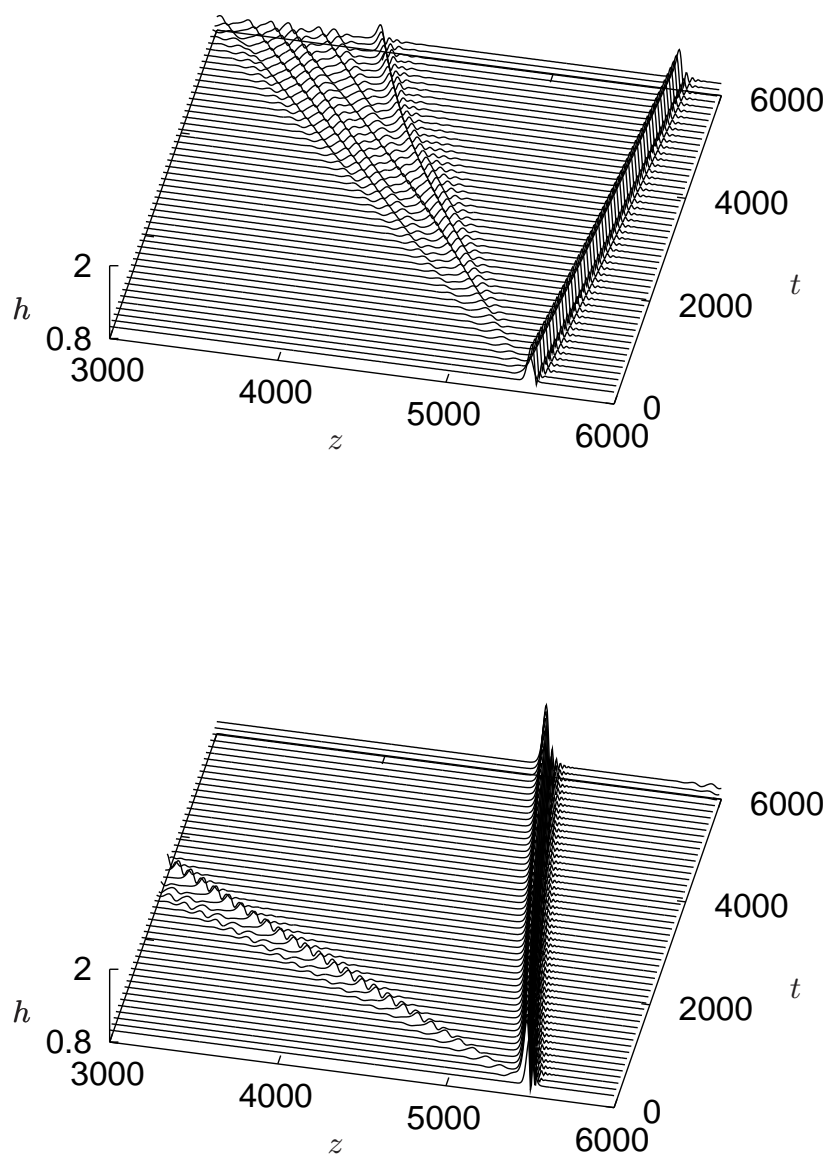

FiguRE 9. Time-evolution of the free surface $h$ obtained from the IBL model in the moving coordinate $z=x-$ ct. The values of the dimensionless groups are $\Gamma=3000$, $\mathrm{Bi}=0.1, \mathcal{E}=1, \phi=1, \beta=\pi / 2, \mathrm{Da}=10000, \mathcal{M}=500, \operatorname{Pr}=1, \mathrm{Sc}=10$ and $\operatorname{Re}=5.085$. Successive curves are separated by $\Delta t=120$. - (a) The initial condition is a Gaussian distribution with amplitude 0.2 at the center of the computational domain and $c=1.34273$, which is the velocity of the traveling wave obtained from the lower branch in figure 5d. - (b) The initial condition is a Gaussian distribution with amplitude 0.4 at the center of the computational domain and $c=1.85428$, which is the velocity of the traveling wave obtained from the upper branch in figure $5 \mathrm{~d}$. 


\section{Conclusion}

We have examined the dynamics of a falling film in the presence of an exothermic chemical reaction. The reactants in the gas above the liquid film, penetrate into the film bulk through its free surface and react as they diffuse, increasing locally the temperature of the liquid. The resulting reaction-diffusion process is fully coupled to the hydrodynamics since the temperature gradients created along the film surface by the reaction generate Marangoni stresses which in turn alter the flow, while the chemical species and consequently the reaction are affected by convection.

The steady solution reveals that the behavior of the system depends strongly on the reaction rate. In particular, for fast reactions a boundary layer is formed beneath the film surface. Asymptotic expressions for the temperature and concentration profiles in the limit of low and high reaction rates are presented as well as an approximation for intermediate reaction rates. A linear stability analysis of the homogeneous steady state shows that the chemical reaction has an overall stabilizing effect on the film whilst heat losses have a destabilizing effect. Furthermore, there can exist an optimum stabilizing reaction rate, with a maximum for the critical Reynolds number as a function of the Damköhler number.

To examine the nonlinear dynamics of the film, several models are introduced. They all rely on a gradient expansion in which the spatio-temporal variation of free surface, concentration and temperature are slow. Concerning the chemical reaction, the same two limiting cases as for the steady solution are considered, namely the low and high reaction rates, as well as the moderate reaction rate. In each case, a longwave model for the free surface and an integral-boundary-layer model for the free surface, wall concentration and interfacial temperature are derived. The latter appears to be more appropriate to account for the nonlinear regime far from the instability onset. Regarding mass transport across the interface, in the slow-reaction case the long-wave approximation revealed that the larger the amplitude of a wave the greater the mass transport. For the fast-reaction case mass transport appears to depend on the film Damköhler number only, and it is hence independent of the flow conditions beneath a wave.

Solitary waves and associated bifurcation diagrams for both longwave and integral-boundary-layer models are constructed as a function of Reynolds number. Like the case of free falling films, the longwave bifurcation diagrams are typically characterized by branch multiplicity and turning points above which solitary waves do not exist. On the other hand, the integral-boundary-layer diagrams are single-valued curves without any turning points and predict the existence of solitary waves for all Reynolds numbers. Again, like the case of free falling films, the presence of turning points for the long-wave models is unphysical and marks the failure of the long-wave approximation to correctly describe the nonlinear regime of the film far from criticality.

Our integral-boundary-layer models are found to be robust in time-dependent computations. Such computations show that for large times, the free surface, wall concentration and interfacial temperature approach a train of coherent structures which resemble the infinite-domain stationary solitary pulses obtained in the bifurcation diagrams.

Acknowledgements. We thank Christian Ruyer-Quil and Benoit Scheid for stimulating discussions. We acknowledge financial support from the Engineering and Physical Sciences Research Council of England through grant no. GR/S01023, an Advanced Research Fellowship, grant no. GR/S49520 and Platform grant EP/E046029. 


\section{A. The functions $a_{F}^{s}$ and $T_{F}^{s}$}

The functions $a_{F}^{s}$ and $T_{F}^{s}$ utilized in $\S 3.1$ are:

$$
\begin{aligned}
a_{F}^{s} & =\frac{y^{2}}{2}-\frac{h^{2}}{2}+\mathcal{D} y^{2}\left(\frac{1-\mathcal{E} \phi}{24} y^{2}+\frac{(2+B h) \phi \mathcal{E} h y}{12(1+B h)}-\frac{h^{2}}{4}\right)+\mathcal{D} \frac{h^{4}}{24}\left(5-\frac{3+B h}{1+B h} \mathcal{E} \phi\right) \\
& +\mathcal{D}^{2} y^{6}\left(\frac{\mathcal{E}^{2} \phi^{2}}{180}-\frac{\mathcal{E} \phi^{2}}{120}-\frac{\mathcal{E} \phi}{90}+\frac{1}{720}\right)+\mathcal{D}^{2} \mathcal{E} \phi h y^{5} \frac{2+B h}{1+B h}\left(\frac{\phi}{40}-\frac{\mathcal{E} \phi}{60}+\frac{1}{60}\right) \\
& +\mathcal{D}^{2} h^{2} y^{4}\left(\left(\frac{2+B h}{1+B h}\right)^{2}(\mathcal{E}-2) \frac{\mathcal{E} \phi^{2}}{96}+\frac{\mathcal{E} \phi}{24}-\frac{1}{48}\right) \\
& +\frac{\mathcal{D}^{2} \mathcal{E} \phi h^{3} y^{3}}{144(1+B h)}\left(\frac{8+5 B h+B^{2} h^{2}}{(1+B h)} \mathcal{E} \phi-20-11 B h\right)+\mathcal{D}^{2} h^{4} \frac{y^{2}}{48}\left(5-\frac{3+B h}{1+B h} \mathcal{E} \phi\right) \\
& +\frac{\mathcal{D}^{2} \mathcal{E} \phi^{2} h^{6}}{(1+B h)^{2}}\left(\frac{1}{24}+\frac{B h}{40}+\frac{B^{2} h^{2}}{240}-\mathcal{E}\left[\frac{5}{72}+\frac{3}{80} B h+\frac{1}{160} B^{2} h^{2}\right]\right) \\
& +\mathcal{D}^{2} h^{6}\left(\frac{11+4 B h}{1+B h} \frac{\mathcal{E} \phi}{80}-\frac{61}{720}\right)
\end{aligned}
$$

and

$$
\begin{aligned}
T_{F}^{s} & =\frac{(2+B h) h y}{2(1+B h)}-\frac{y^{2}}{2}+\mathcal{D} y^{2}\left(\frac{\mathcal{E} \phi-1}{24} y^{2}-\frac{(2+B h) \phi \mathcal{E} h y}{12(1+B h)}+\frac{h^{2}}{4}\right) \\
& +\mathcal{D} h^{3} y\left(\frac{\left(B^{2} h^{2}+5 B h+8\right) \phi \mathcal{E}}{24(1+B h)^{2}}-\frac{8+5 B h}{24(1+B h)}\right) \\
& -\mathcal{D}^{2} y^{5}\left(y\left(\frac{1}{720}-\frac{\mathcal{E} \phi}{90}-\frac{\mathcal{E} \phi^{2}}{120}+\frac{\mathcal{E}^{2} \phi^{2}}{180}\right)+h \frac{2+B h}{1+B h}\left(\frac{1}{60}+\frac{\phi}{40}-\frac{\mathcal{E} \phi}{60}\right) \mathcal{E} \phi\right) \\
& +\mathcal{D}^{2} h^{2} \frac{y^{2}}{48}\left(y^{2}\left(1-2 \mathcal{E} \phi-\frac{(2+B h)^{2}}{2(1+B h)^{2}}(\mathcal{E}-2) \mathcal{E} \phi^{2}\right)+\frac{3+B h}{1+B h} \mathcal{E} \phi h^{2}-5 h^{2}\right) \\
& +\mathcal{D}^{2} \mathcal{E} \phi h^{3} y^{3}\left(-\frac{\mathcal{E}\left(B^{2} h^{2}+5 B h+8\right) \phi}{144(1+B h)^{2}}+\frac{(11 B h+20)}{144(1+B h)}\right) \\
& +\frac{\mathcal{D}^{2} \mathcal{E} \phi^{2} h^{5}}{(1+B h)^{3}} y\left(\left(\frac{1}{5}+\frac{113}{720} B h+\frac{B^{2} h^{2}}{20}+\frac{B^{3} h^{3}}{160}\right) \mathcal{E}-\frac{2}{15}-\frac{B h}{10}-\frac{B^{2} h^{2}}{30}-\frac{B^{3} h^{3}}{240}\right) \\
& -\mathcal{D}^{2} h^{5} y\left(\frac{11}{40}+\frac{9}{40} B h+\frac{1}{20} h^{2} B^{2}\right) \frac{\mathcal{E} \phi}{(1+B h)^{2}}+\mathcal{D}^{2} h^{5} y \frac{96+61 B h}{720(1+B h)} .
\end{aligned}
$$

The subscript $F$ and superscript $s$ are used to denote 'flat film' and 'slow reaction', respectively. Indeed, $h=1$ in these functions gives the flat film concentration and temperature distributions for the slow reaction case. On the other hand, for $h \rightarrow h(x, t)$ these functions appear in the LWE concentration and temperature distributions given in Appendix B. 


\section{B. Long-wave functions - Slow reaction}

The functions $a_{L}^{s}$ and $T_{L}^{s}$ in $\S 5.1$ are given by

$$
\begin{aligned}
& a_{L}^{s}=a_{F}^{s}-\operatorname{ReSch}_{x}\left(\frac{3}{40} y^{5}-\frac{h}{8} y^{4}+\frac{h^{2}}{2} y^{3}-\frac{3}{2} h^{3} y^{2}+\frac{21}{20} h^{5}\right) \\
& +\operatorname{ReSc} h_{x} y^{7}\left(\frac{(2+B h) B h}{336(1+B h)^{2}} \mathcal{E} \phi-\frac{13}{1680}\right) \\
& +\operatorname{ReSc} \mathcal{D} h h_{x} y^{5}\left(\frac{7 y}{240}-\frac{2+5 B h+B^{2} h^{2}}{240(1+B h)^{2}} \mathcal{E} \phi y-\frac{h}{16}-\frac{2+2 B h+B^{2} h^{2}}{40(1+B h)^{2}} \mathcal{E} \phi h\right) \\
& +\operatorname{ReSc} \mathcal{D} h^{3} h_{x} y^{3}\left(\frac{7 y}{48}+\frac{6+7 B h+2 B^{2} h^{2}}{96(1+B h)^{2}} \mathcal{E} \phi y+\frac{5 h}{12}-\frac{6+7 B h+2 B^{2} h^{2}}{24(1+B h)^{2}} \mathcal{E} \phi h\right) \\
& +\operatorname{ReSc} h^{5} h_{x}\left(\frac{6+7 B h+2 B^{2} h^{2}}{8(1+B h)^{2}} \mathcal{E} \phi y^{2}-\frac{71}{40} y^{2}+\frac{351}{280} h^{2}\right) \\
& -\operatorname{ReSc} h^{7} h_{x} \frac{1778+2071 B h+584 B^{2} h^{2}}{3360(1+B h)^{2}} \mathcal{E} \phi+\operatorname{Re}^{2} \operatorname{Sc}^{2} h_{x x}\left(\frac{y^{9}}{640}-\frac{33 h y^{8}}{4480}+\frac{9 h^{2} y^{7}}{280}\right) \\
& -\operatorname{Re}^{2} \operatorname{Sc}^{2} h_{x x}\left(\frac{11}{80} h^{3} y^{6}-\frac{3}{10} h^{4} y^{5}+\frac{39}{160} h^{5} y^{4}+\frac{21}{40} h^{6} y^{3}-\frac{63}{40} h^{7} y^{2}+\frac{2229}{2240} h^{9}\right) \\
& +\operatorname{Re}^{2} \operatorname{Sc}^{2} h_{x}^{2}\left(\frac{3 y^{8}}{448}+\frac{3 h y^{7}}{80}-\frac{23}{80} y^{6} h^{2}+\frac{3}{4} y^{5} h^{3}-\frac{39}{32} y^{4} h^{4}-\frac{21}{8} y^{3} h^{5}+\frac{441}{40} y^{2} h^{6}\right) \\
& -\operatorname{Re}^{2} \operatorname{Sc}^{2} h_{x}^{2} h^{8} \frac{17221}{2240}
\end{aligned}
$$

and

$$
\begin{aligned}
T_{L}^{s} & =T_{F}^{s}+\frac{\operatorname{RePr} h^{4} h_{x} y}{40(1+B h)^{3}}(2+B h)\left(6 B^{2} h^{2}+20+15 B h\right) \\
& +\frac{\operatorname{RePr} h h_{x} y^{3}}{(1+B h)^{2}}\left(\frac{3}{80}(2+B h) B y^{2}+\left(2+B h+B^{2} h^{2}\right) \frac{y}{16}-\left(2+2 B h+B^{2} h^{2}\right) \frac{h}{4}\right) \\
& +\operatorname{ReSc} \mathcal{D} h_{x} y\left(\frac{y^{6}}{560}-\frac{h y^{5}}{240}+\frac{h^{2} y^{4}}{40}-\frac{h^{3} y^{3}}{8}+\frac{21 h^{5} y}{40}-\frac{(710 B h+1113) h^{6}}{1680(1+B h)}\right),
\end{aligned}
$$

where the functions $a_{F}^{s}$ and $T_{F}^{s}$ are defined in Appendix A.

The $u$-velocity is given by:

$$
\begin{aligned}
& u=\left(1-\cot \beta h_{x}+\operatorname{Weh}_{x x x}\right)\left(h y-\frac{y^{2}}{2}\right)+\operatorname{Reh}_{x}\left(h-\frac{y}{2}\right)\left(h^{2}+\frac{h y}{2}-\frac{y^{2}}{4}\right) y h \\
& -\mathcal{M} h_{x} \frac{h(2+B h) y}{2(1+B h)^{2}}+\frac{\mathcal{M D} h^{3} h_{x} y}{(1+B h)^{3}}\left(\frac{1}{2}+\frac{7 B h}{8}+\frac{3 B^{2} h^{2}}{8}-\left(\frac{5}{6}+\frac{5 B h}{8}+\frac{B^{2} h^{2}}{8}\right) \mathcal{E} \phi\right) \\
& +\frac{403}{1680} \operatorname{ReSc} \mathcal{M D} y\left(\frac{h^{7} h_{x}}{1+B h}\right)_{x}-\frac{1}{80} \operatorname{RePr} \mathcal{M} y\left(\frac{\left(50+41 B h+13 B^{2} h^{2}\right) h^{5} h_{x}}{(1+B h)^{3}}\right)_{x} \\
& +\frac{\mathcal{M D}^{2} h^{5} h_{x} y}{(1+B h)^{4}} \mathcal{E} \phi^{2}\left(\frac{11}{20}+\frac{61}{120} B h+\frac{B^{2} h^{2}}{6}+\frac{B^{3} h^{3}}{48}\right) \\
& -\frac{\mathcal{M D}^{2} h^{5} h_{x} y}{(1+B h)^{4}} \mathcal{E}^{2} \phi^{2}\left(\frac{47}{60}+\frac{89}{120} B h+\frac{B^{2} h^{2}}{4}+\frac{B^{3} h^{3}}{32}\right) \\
& +\frac{\mathcal{M D}^{2} h^{5} h_{x} y}{(1+B h)^{4}} \mathcal{E} \phi\left(\frac{33}{40}+\frac{131}{80} B h+B^{2} h^{2}+\frac{3}{16} B^{3} h^{3}\right) \\
& -\frac{\mathcal{M D}^{2} h^{5} h_{x} y}{(1+B h)^{4}}\left(\frac{7}{24}+\frac{119 B h}{144}+\frac{7 B^{2} h^{2}}{9}+\frac{35 B^{3} h^{3}}{144}\right) \text {. }
\end{aligned}
$$


Finally, the flow rate $q^{s}$ in the streamwise direction is given by:

$$
\begin{aligned}
& q^{s}=\frac{h^{3}}{3}\left(1-\cot \beta h_{x}+\operatorname{We} h_{x x x}\right)+\frac{2}{5} \operatorname{Re}^{6} h_{x}-\frac{\mathcal{M}(2+B h) h^{3} h_{x}}{4(1+B h)^{2}} \\
& +\frac{\mathcal{M D} h^{5} h_{x}}{(1+B h)^{3}}\left(\frac{1}{4}+\frac{7 B h}{16}+\frac{3 B^{2} h^{2}}{16}-\left(\frac{5}{12}+\frac{5 B h}{16}+\frac{B^{2} h^{2}}{16}\right) \mathcal{E} \phi\right)
\end{aligned}
$$

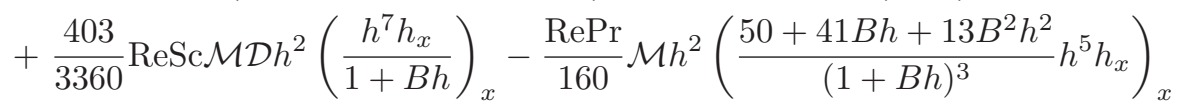

$$
\begin{aligned}
& +\frac{\mathcal{D}^{2} \mathcal{M} h^{7} h_{x}}{(1+B h)^{4}} \mathcal{E} \phi^{2}\left(\frac{11}{40}+\frac{61}{240} B h+\frac{1}{12} B^{2} h^{2}+\frac{1}{96} B^{3} h^{3}\right) \\
& -\frac{\mathcal{D}^{2} \mathcal{M} h^{7} h_{x}}{(1+B h)^{4}} \mathcal{E}^{2} \phi^{2}\left(\frac{47}{120}+\frac{89}{240} B h+\frac{1}{8} B^{2} h^{2}+\frac{1}{64} B^{3} h^{3}\right) \\
& +\frac{\mathcal{D}^{2} \mathcal{M} h^{7} h_{x}}{(1+B h)^{4}} \mathcal{E} \phi\left(\frac{33}{80}+\frac{131}{160} B h+\frac{1}{2} B^{2} h^{2}+\frac{3}{32} B^{3} h^{3}\right) \\
& -\frac{\mathcal{D}^{2} \mathcal{M} h^{7} h_{x}}{(1+B h)^{4}}\left(\frac{7}{48}+\frac{119}{288} B h+\frac{7}{18} B^{2} h^{2}+\frac{35}{288} B^{3} h^{3}\right) \text {. }
\end{aligned}
$$

\section{Long-wave functions - Fast reaction}

For a fast reaction, the long wave expansion in $\S 5.2$ yields the following solutions for the leading order velocity, concentration and temperature solutions

$$
u_{0,0}=h y-\frac{y^{2}}{2}, \quad a_{0,0}=\frac{1}{\mathcal{D}}\left(e^{\Pi \mathcal{D}^{1 / 2}(y-h)}-1\right), \quad T_{0,0}=\frac{B+\Pi \mathcal{D}^{1 / 2}}{\mathcal{D}(1+B h)} y-\frac{1}{\mathcal{D}} e^{\Pi \mathcal{D}^{1 / 2}(y-h)} .
$$

The first order corrections are:

$$
a_{0,1}=\frac{3 \operatorname{ReSc} h_{x}}{4 \Pi \mathcal{D}^{3 / 2}} e^{\Pi \mathcal{D}^{1 / 2}(y-h)} h(h-y)\left(\mathcal{D}^{1 / 2} \Pi(y-h)-1\right),
$$

and

$$
\begin{aligned}
T_{0,1} & =-\frac{3 \operatorname{ReSch} h_{x} y}{4 \Pi^{2} \mathcal{D}^{2}(1+B h)}\left(3 \Pi \mathcal{D}^{1 / 2}+8 B\right) \\
& +\frac{\operatorname{RePr} h_{x} y^{3}}{40 \mathcal{D}(1+B h)^{2}}\left(\Pi \mathcal{D}^{1 / 2}+B\right)\left(3 B y^{2}-5(1+3 B h) y+20 B h^{2}\right) \\
& +\frac{\operatorname{RePr} h_{x} h y}{\mathcal{D}(1+B h)}\left(\left[\frac{1}{2}-\frac{B h}{4}-\frac{B^{2} h^{2}}{5}\right] \frac{\Pi \mathcal{D}^{1 / 2}+B}{(1+B h)^{2}} h^{2}+\frac{3}{\Pi^{2} \mathcal{D}}\left(\Pi \mathcal{D}^{1 / 2}+2 B\right)\right) \\
& +\frac{3 \operatorname{ReSc} h_{x}}{4 \Pi^{2} \mathcal{D}^{2}} h\left(\Pi^{2} \mathcal{D}(h-y)^{2}+5 \Pi \mathcal{D}^{1 / 2}(h-y)+8\right) e^{\Pi \mathcal{D}^{1 / 2}(y-h)} \\
& +\frac{3 \operatorname{RePr} h_{x}}{\Pi^{2} \mathcal{D}^{2}} h\left(\Pi \mathcal{D}^{1 / 2}(y-h)-2\right) e^{\Pi \mathcal{D}^{1 / 2}(y-h)} .
\end{aligned}
$$

The $\mathcal{O}(\epsilon) u$-velocity term is,

$$
\begin{aligned}
u_{1,0} & =\frac{1}{8} \operatorname{Re} h h_{x} y(y-2 h)\left(y^{2}-2 h y-4 h^{2}\right)+\left(\cot \beta h_{x}-\operatorname{We}_{x x x}\right) y \frac{y-2 h}{2} \\
& -\frac{\mathcal{M}\left(\Pi \mathcal{D}^{1 / 2}+B\right)}{\mathcal{D}(1+B h)^{2}} h_{x} y
\end{aligned}
$$


the $\mathcal{O}(\epsilon \Delta) u$-velocity term is,

$$
\begin{aligned}
u_{1,1} & =\frac{3 \operatorname{ReSc} \mathcal{M} y}{4 \Pi^{2} \mathcal{D}^{2}}\left[\left(3 h \Pi \mathcal{D}^{1 / 2}-8\right) \frac{h h_{x}}{(1+B h)^{2}}\right]_{x} \\
& +\frac{\operatorname{RePr} \mathcal{M} y}{\Pi^{2} \mathcal{D}^{2}}\left[\left(\frac{h^{3}(7 B h-15)}{40(1+B h)^{2}} \Pi^{2} \mathcal{D}\left(\Pi \mathcal{D}^{1 / 2}+B\right)-3 \Pi \mathcal{D}^{1 / 2} h+6\right) \frac{h h_{x}}{1+B h}\right]_{x}
\end{aligned}
$$

Finally, the flow rate $q^{f}$ in the streamwise direction is given by:

$$
\begin{aligned}
q^{f} & =\frac{h^{3}}{3}\left(1-\cot \beta h_{x}+\text { We } h_{x x x}\right)+\frac{2}{5} \operatorname{Re}^{6} h_{x}-\mathcal{M} \frac{\Pi \mathcal{D}^{1 / 2}+B}{2 \mathcal{D}(1+B h)^{2}} h^{2} h_{x} \\
& +\frac{3 \operatorname{ReSc} \mathcal{M} h^{2}}{8 \Pi^{2} \mathcal{D}^{2}}\left[\left(3 h \Pi \mathcal{D}^{1 / 2}-8\right) \frac{h h_{x}}{(1+B h)^{2}}\right]_{x} \\
& +\frac{\operatorname{RePr} \mathcal{M} h^{2}}{2 \Pi^{2} \mathcal{D}^{2}}\left[\left(\frac{h^{3}(7 B h-15)}{40(1+B h)^{2}} \Pi^{2} \mathcal{D}\left(\Pi \mathcal{D}^{1 / 2}+B\right)-3 \Pi \mathcal{D}^{1 / 2} h+6\right) \frac{h h_{x}}{1+B h}\right]_{x} .
\end{aligned}
$$

\section{Rescaled long-wave functions}

For the slow reaction case (LWE-S) the functions $\mathcal{A}^{s}, \mathcal{B}^{s}$ and $\mathcal{C}^{s}$ are given by:

$$
\begin{gathered}
\mathcal{A}^{s}=\frac{h^{3}}{\mathrm{We}^{\frac{1}{3}}}\left(\frac{2}{5} \operatorname{Re} h^{3}-\frac{\cot \beta}{3}-\mathcal{M} G^{s}\right) \\
\mathcal{B}^{s}=\mathcal{M} \frac{\operatorname{Re}^{6}}{\mathrm{We}^{2 / 3}}\left(\frac{403}{3360} \operatorname{Sc}^{2} h^{2} \frac{7+6 B h}{(1+B h)^{2}}-\operatorname{Pr} \frac{125+173 B h+107 B^{2} h^{2}+26 B^{3} h^{3}}{80(1+B h)^{4}}\right) \\
\mathcal{C}^{s}=\mathcal{M} \frac{\operatorname{Re}^{7}}{\mathrm{We}^{2 / 3}}\left(\frac{403}{3360} \frac{\mathrm{Sc} \mathcal{D} h^{2}}{(1+B h)}-\operatorname{Pr} \frac{50+41 B h+13 B^{2} h^{2}}{160(1+B h)^{3}}\right),
\end{gathered}
$$

where,

$$
\begin{aligned}
G^{s} & =\frac{2+B h}{4(1+B h)^{2}}-\frac{\mathcal{D} h^{2}}{16}\left(\frac{4+3 B h}{(1+B h)^{2}}-\mathcal{E} \phi \frac{20+15 B h+3 B^{2} h^{2}}{3(1+B h)^{3}}\right)+\frac{7 \mathcal{D}^{2} h^{4}(6+5 B h)}{288(1+B h)^{2}} \\
& -\mathcal{D}^{2} \frac{\mathcal{E} \phi h^{4}}{160}\left(\frac{66+65 B h+15 B^{2} h^{2}}{(1+B h)^{3}}+\phi \frac{132+122 B h+40 B^{2} h^{2}+5 B^{3} h^{3}}{3(1+B h)^{4}}\right) \\
& +\frac{\mathcal{D}^{2} \mathcal{E}^{2} \phi^{2} h^{4}}{960(1+B h)^{4}}\left(376+356 B h+120 B^{2} h^{2}+15 B^{3} h^{3}\right) .
\end{aligned}
$$

For the fast reaction case (LWE-F) the functions $\mathcal{A}^{f}, \mathcal{B}^{f}$ and $\mathcal{C}^{f}$ are given by:

$$
\begin{gathered}
\mathcal{A}^{f}=\frac{h^{3}}{\mathrm{We}^{\frac{1}{3}}}\left(\frac{2}{5} \operatorname{Re}^{3}-\frac{\cot \beta}{3}-\mathcal{M} G^{f}\right) \\
\mathcal{B}^{f}=\frac{6 \mathcal{M R e S c h}{ }^{2}\left(3 \Pi \mathcal{D}^{1 / 2} h+4 B h-4\right)}{8 \Pi^{2} \mathcal{D}^{2} \mathrm{We}^{2 / 3}(1+B h)^{3}}+\frac{\mathcal{M} \operatorname{RePr} h^{4}}{40 \mathcal{D W e} \mathrm{We}^{2 / 3}}\left(33 \frac{1-\Pi \mathcal{D}^{1 / 2} h}{(1+B h)^{4}}-\frac{29+4 \Pi \mathcal{D}^{1 / 2} h}{(1+B h)^{3}}\right) \\
+\frac{\mathcal{M} \operatorname{RePr}^{2}}{40 \Pi^{2} \mathcal{D}^{2} \mathrm{We}^{2 / 3}}\left(\Pi \mathcal{D}^{1 / 2} h \frac{7 \Pi \mathcal{D}^{1 / 2} h-120}{1+B h}+120 \frac{2-\Pi \mathcal{D}^{1 / 2} h}{(1+B h)^{2}}+\Pi^{2} \mathcal{D}^{2} \frac{7 \Pi \mathcal{D}^{1 / 2} h-11}{(1+B h)^{2}}\right) \\
\mathcal{C}^{f}=\frac{3 \mathcal{M} \operatorname{ReSc}\left(3 \Pi \mathcal{D}^{1 / 2} h-8\right) h^{3}}{8 \Pi^{2} \mathcal{D}^{2} \mathrm{We}^{2 / 3}(1+B h)^{2}} \\
+\frac{\mathcal{M R e P r}^{3}}{\Pi^{2} \mathcal{D}^{2} \mathrm{We}^{2 / 3}(1+B h)}\left(\frac{h^{3}(7 B h-15)}{80(1+B h)^{2}} \Pi^{2} \mathcal{D}\left(\Pi \mathcal{D}^{1 / 2}+B\right)-3 \Pi \mathcal{D}^{1 / 2} h+6\right),
\end{gathered}
$$


where,

$$
G^{f}=\frac{\Pi \mathcal{D}^{1 / 2}+B}{2 \mathcal{D} h(1+B h)^{2}} .
$$

\section{E. Additional functions involved in IBL}

The functions $J_{e a}$ and $J_{e t}$ involved in the weighted integral approach for a slow chemical reaction are given by:

$$
\begin{aligned}
J_{e a} & =\frac{1}{2}+\mathcal{D}\left[\frac{2}{5} \alpha+\left(\frac{11}{40}+\frac{7 B h}{80}\right) \phi \mathcal{E} \theta\right]+\mathcal{D}^{2} \mathcal{E} \phi \theta \alpha\left(\frac{27}{140}+\frac{19 B h}{280}\right) \\
& +\mathcal{D}^{2} \mathcal{E}(\mathcal{E}-2) \phi^{2} \theta^{2}\left(\frac{27}{280}+\frac{31}{560} B h+\frac{B^{2} h^{2}}{112}\right)
\end{aligned}
$$

and

$$
\begin{aligned}
J_{e t} & =\frac{1}{2}+\frac{B h}{8}+\mathcal{D}\left[\left(\frac{11}{40}+\frac{7 B h}{80}\right) \alpha+\left(\frac{2}{5}+\frac{7}{40} B h+\frac{B^{2} h^{2}}{40}\right) \phi \mathcal{E} \theta\right] \\
& +\mathcal{D}^{2} \mathcal{E} \phi \theta \alpha\left(\frac{27}{140}+\frac{31}{280} B h+\frac{B^{2} h^{2}}{56}\right) \\
& +\mathcal{D}^{2} \mathcal{E}(\mathcal{E}-2) \phi^{2} \theta^{2}\left(\frac{6}{35}+\frac{57}{560} B h+\frac{3 B^{2} h^{2}}{112}+\frac{3 B^{3} h^{3}}{1120}\right) .
\end{aligned}
$$

The functions $\mathcal{P}_{i}$ for $\mathrm{i}=1$ to 4 involved in the weighted integral approach for a fast chemical reaction are given by:

$$
\begin{gathered}
\mathcal{P}_{1}=1+\frac{2 B}{\mathcal{D}^{\prime}}-\frac{6+6 B h-B^{2} h^{2}}{\mathcal{D}^{\prime 2} h^{2}}+\frac{15+6 B h-9 B^{2} h^{2}}{2 \mathcal{D}^{\prime 3} h^{3}}+6 \frac{(1+B h) B}{\mathcal{D}^{\prime 4} h^{3}} \\
\mathcal{P}_{2}=\frac{3}{20}+\frac{3 B}{10 \mathcal{D}^{\prime}}-\frac{20+20 B h-3 B^{2} h^{2}}{20 \mathcal{D}^{\prime 2} h^{2}}+\frac{5+2 B h-3 B^{2} h^{2}}{4 \mathcal{D}^{\prime 3} h^{3}} \\
+\frac{2+3 B h+B^{2} h^{2}}{\mathcal{D}^{\prime 4} h^{4}}-\frac{49+34 B h-15 B^{2} h^{2}}{8 \mathcal{D}^{\prime 5} h^{5}}-6 \frac{(1+B h) B h}{\mathcal{D}^{\prime 6} h^{6}} \\
\mathcal{P}_{3}=-\frac{3}{20}+\frac{5+B h}{5 \mathcal{D}^{\prime} h}-3 \frac{25-3 B^{2} h^{2}}{40 \mathcal{D}^{\prime 2} h^{2}}-\frac{27+36 B h+5 B^{2} h^{2}}{8 \mathcal{D}^{\prime 3} h^{3}} \\
+\frac{277+160 B h-45 B^{2} h^{2}}{16 \mathcal{D}^{\prime 4} h^{4}}-\frac{147-172 B h-207 B^{2} h^{2}}{16 \mathcal{D}^{\prime 5} h^{5}}-\frac{(9+6 B h) B h}{\mathcal{D}^{\prime 6} h^{6}} \\
\mathcal{P}_{4}= \\
+9 \frac{7 \mathcal{D}^{\prime} h}{5}+\frac{33+14 B h}{5}-\frac{300+114 B h-7 B^{2} h^{2}}{5 \mathcal{D}^{\prime} h} \\
-3 \frac{195+10 B h-B^{2} h^{2}}{5 \mathcal{D}^{\prime 2} h^{2}}+3 \frac{9+50 B h+17 B^{2} h^{2}}{\mathcal{D}^{\prime 3} h^{3}} \\
2 \mathcal{D}^{\prime 4} h^{4}
\end{gathered}
$$

\section{F. Moderate reaction}

The slow and fast reaction cases can be analyzed rigorously, however, this is not the case for the moderate reaction rate, i.e. when $\mathcal{D} \sim \mathcal{O}(1)$. Here we present an approximate analytical solution for the latter case. Since the resulting equations are rather involved we refrain from solving them numerically. 


\section{F.1. Padé-like approximation to the Arrhenius term}

For the moderate reaction case the Arrhenius term, written as

$$
A(r)=e^{\frac{\mathcal{E} r}{1+r}}
$$

where $r=\mathcal{D} \phi T$, can be difficult if not impossible to deal with analytically. If $r$ or $\mathcal{E}$ are small then Taylor expansions of this term yield:

$$
A=1+\mathcal{E} r+\mathcal{E}(\mathcal{E}-2) \frac{r^{2}}{2}+\mathcal{O}\left(r^{3}\right), \quad A=1+\frac{\mathcal{E} r}{1+r}+\frac{\mathcal{E}^{2} r^{2}}{2(1+r)^{2}}+\mathcal{O}\left(\mathcal{E}^{3}\right) .
$$

However, when $r$ and $\mathcal{E}$ are $\mathcal{O}(1)$, both of these expansions diverge.

A better approximation can be obtained by employing instead a Padé-like approximation around $r=0$. If we use $N=M=1$, where $N$ and $M$ are respectively the order of the polynomial in the numerator and denominator of the approximation, we have:

$$
A(r) \simeq \frac{1+(1+\mathcal{E} / 2) r}{1+(1-\mathcal{E} / 2) r}
$$

This increases the radius of convergence over the Taylor expansion about small $r$, when $\mathcal{E}$ is small, but it is clear that at $\frac{\mathcal{E} r}{1+r}=2$ the approximation has failed with a singularity. Notice the Padé approximation $e^{r} \simeq \frac{2+r}{2-r}$ which has a $20 \%$ error by $r=1.2$ and a singularity at $r=2$. Even increasing $N$ and $M$ from 1 to 9 only extends the region of a $20 \%$ error up to $r=8.1$ with a singularity around $r=9.1$.

Since $r>0,0 \leq \frac{r}{1+r} \leq 1$, thus $1 \leq A(r) \leq e^{\mathcal{E}}$. Hence, $A$ remains bounded $\forall r$ when $\mathcal{E}$ is fixed, so an attempt to increase the radius of convergence of $\mathcal{E}$ shall be made. In order to apply a Padé-like approximation using $\mathcal{E}$ as the variable we need to explicitly choose the functional form for the terms involving $\mathcal{E}$. We shall continue with the expression

$$
R=\frac{p_{0}(\mathcal{E})+p_{1}(\mathcal{E}) r}{1+q_{1}(\mathcal{E}) r}
$$

which was the rational form with $N=M=1$ when $r$ was employed. Noting that $f$ is monotonically increasing in $r$ and $\mathcal{E}$, we specify the $\mathcal{E}$ dependence in the form:

$$
R=\frac{\hat{p}_{0} e^{a_{0} \mathcal{E}}+\hat{p}_{1} e^{a_{1} \mathcal{E}} r}{1+\hat{q}_{1} e^{b_{1} \mathcal{E}} r}
$$

Satisfying then equality of $f$ and $R$ and their first two derivatives at $\mathcal{E}=0$ we obtain $\hat{p}_{0}=1, \hat{p}_{1}=\hat{q}_{1}=1$, $a_{0}=0, a_{1}=1 / 2$ and $b_{1}=-1 / 2$ to yield:

$$
A(r, \mathcal{E}) \simeq \frac{1+e^{\mathcal{E} / 2} r}{1+e^{-\mathcal{E} / 2} r}
$$

In the limit of small $\mathcal{E}$, the maximum absolute error given by this expression is $\frac{25}{36 \sqrt{3}} \mathcal{E}^{3} \%$. Comparing numerically the Arrhenius term with expression (F.4) we find that for $\mathcal{E}=0.3,1$ and 3 the approximate maximum absolute errors are $0.01 \%, 0.4 \%$ and $10.2 \%$, respectively. It is important that $\mathcal{E}$ is at most $\mathcal{O}(1)$ since the expression in (F.4) very quickly becomes an inappropriate approximation as $\mathcal{E}$ increases.

\section{F.2. Flat film solution}

In the large- $\mathcal{D}$-limit the concentration and temperature had exponential profiles whilst in the small- $\mathcal{D}$ limit they had quadratic profiles. By utilizing a cosh test function the quadratic terms can be obtained in a suitable limit. Hence, we combine a linear test function with a cosh test function in an attempt to yield both exponential and quadratic profiles in the appropriate limits of $\mathcal{D}$. 
We suppose that the concentration and temperature can be written in the form:

$$
a=r_{0}+r_{1} y+r_{2} \cosh \left(r_{3} y\right) \text { and } T=s_{0}+s_{1} y+s_{2} \cosh \left(s_{3} y\right)
$$

The sum of the concentration and energy equations yield $a_{y y}+T_{y y} \equiv 0$, thus we require that $s_{2}=-r_{2}$ and $s_{3}=r_{3}$. The four boundary conditions require that $r_{1}=0, r_{0}=-r_{2} \cosh \left(r_{3} h\right), s_{0}=r_{2}$ and $s_{1}=r_{2}\left[r_{3} \sinh \left(r_{3} h\right)+B\left(\cosh \left(r_{3} h\right)-1\right)\right] /(1+B h)$ to yield $a=a_{F}^{m}$ and $T=T_{F}^{m}$ where

$$
a_{F}^{m}=r_{2}\left(\cosh \left(r_{3} y\right)-\cosh \left(r_{3} h\right)\right)
$$

and

$$
T_{F}^{m}=r_{2}\left(1-\cosh \left(r_{3} y\right)+\frac{y}{1+B h}\left[r_{3} \sinh \left(r_{3} h\right)+B\left(\cosh \left(r_{3} h\right)-1\right)\right]\right) .
$$

The superscript $m$ denotes a moderate reaction rate. These expressions satisfy all of the boundary conditions along with the sum of the heat and mass transport equation. The only two unknowns remaining are $r_{2}$ and $r_{3}$. We require these expressions to be asymptotically equivalent to the solutions obtained in the limits of $\mathcal{D} \rightarrow 0$ and $\mathcal{D} \rightarrow \infty$.

As $\mathcal{D} \rightarrow 0$ the leading order terms from equation (A.1) are:

$$
a=\frac{y^{2}}{2}-\frac{h^{2}}{2}+\mathcal{O}(\mathcal{D}) \quad \text { and } \quad T=\frac{(2+B h) h y}{2(1+B h)}-\frac{y^{2}}{2}+\mathcal{O}(\mathcal{D}) .
$$

As $r_{3} \rightarrow 0$ the expressions (F.5) become:

$$
a=r_{2} r_{3}^{2}\left(\frac{y^{2}}{2}-\frac{h^{2}}{2}\right)+\mathcal{O}\left(r_{3}^{4}\right) \quad \text { and } \quad T=r_{2} r_{3}^{2}\left(\frac{(2+B h) h y}{2(1+B h)}-\frac{y^{2}}{2}\right)+\mathcal{O}\left(r_{3}^{4}\right) .
$$

Thus, as $r_{3} \rightarrow 0$, the $\mathcal{D} \rightarrow 0$ limit can be obtained when $r_{2} r_{3}^{2} \rightarrow 1$.

As $\mathcal{D} \rightarrow \infty$ the leading-order terms from equation (3.3) are

$$
a=\mathcal{D}^{-1}\left(e^{\Pi \mathcal{D}^{1 / 2}(y-h)}-1\right) \quad \text { and } \quad T=\frac{1}{\mathcal{D}}\left[\frac{\Pi \mathcal{D}^{1 / 2}+B}{1+B h} y-e^{\Pi \mathcal{D}^{1 / 2}(y-h)}\right]
$$

where $\Pi=e^{\mathcal{E} / 2}$. As $r_{3} \rightarrow \infty$ the expressions (F.5) become:

$$
a=\frac{1}{2} r_{2} e^{r_{3} h}\left(e^{r_{3}(y-h)}-1\right) \quad \text { and } \quad T=\frac{1}{2} r_{2} e^{r_{3} h}\left(\frac{r_{3}+B}{1+B h} y-e^{r_{3}(y-h)}\right) .
$$

Thus as $r_{3} \rightarrow \infty$, the $\mathcal{D} \rightarrow \infty$ limit can be obtained when $r_{3} \rightarrow \Pi \mathcal{D}^{1 / 2}$ and $r_{2} \rightarrow 2 \mathcal{D}^{-1} e^{-\Pi \mathcal{D}^{1 / 2} h}$. For convenience we can choose

$$
r_{3}=\Pi \mathcal{D}^{1 / 2}
$$

then the above conditions are:

$$
\lim _{\mathcal{D} \rightarrow 0} r_{2} \rightarrow \Pi^{-2} \mathcal{D}^{-1} \text { and } \quad \lim _{\mathcal{D} \rightarrow \infty} r_{2} \rightarrow 2 \mathcal{D}^{-1} e^{-\Pi \mathcal{D}^{1 / 2} h}
$$

These two limits are insufficient to uniquely determine $r_{2}$. However, substituting the profiles for $a$ and $T$ into the concentration and energy equations and averaging across the film allows $r_{2}$ to be determined. Although a Galerkin approach could be applied here, the mass transport and heat transport equation would yield different solutions for $r_{2}$, hence, for uniqueness, the unity weight function is applied, so that the heat and mass transport equations are equivalent. We note that we have averaged across the film 
thickness because the test functions defined in equation (F.5) do not satisfy the differential equations exactly.

The mass transport equation is:

$$
a_{y y}=(1+\mathcal{D} a) e^{\frac{\mathcal{E} \mathcal{D} \phi T}{1+\mathcal{D} \phi T}} .
$$

However, the Arrhenius term can not be integrated analytically, hence, we shall employ the Padé-like approach described in $\S$ F.1 to approximate the Arrhenius term. We then use:

$$
e^{\frac{\mathcal{E} x}{1+x}} \simeq \frac{1+\Pi x}{1+\Pi^{-1} x} .
$$

This allows the mass transport equation to be written as:

$$
a_{y y}\left(1+\Pi^{-1} \phi \mathcal{D} T\right)=(1+\mathcal{D} a)(1+\Pi \phi \mathcal{D} T) .
$$

Integrating then the equation over the film thickness with a weight function of unity and requiring this averaged equation to be zero we obtain:

$$
0=\frac{1}{h} \int_{0}^{h}(1+\mathcal{D} a)(1+\Pi \phi \mathcal{D} T)-a_{y y}\left(1+\Pi^{-1} \phi \mathcal{D} T\right) d y .
$$

This integral yields a quadratic equation in $r_{2}$ given by

$$
0=1+I_{1} r_{2}+I_{2} r_{2}^{2}
$$

where

$$
I_{1}=\frac{1}{h r_{2}} \int_{0}^{h}\left(\mathcal{D} a+\Pi \phi \mathcal{D} T-a_{y y}\right) d y \quad \text { and } \quad I_{2}=\frac{1}{h r_{2}^{2}} \mathcal{D} \phi \int_{0}^{h} T\left(\mathcal{D} \Pi a-\Pi^{-1} a_{y y}\right) d y .
$$

These integrals are evaluated to give

$$
\begin{gathered}
I_{1}=-\frac{\mathcal{D}^{1 / 2}}{2 \Pi h}\left(\left(\Pi \mathcal{D}^{1 / 2} h+\Pi^{2}-1\right) e^{\Pi \mathcal{D}^{1 / 2} h}+\left(\Pi \mathcal{D}^{1 / 2} h+1-\Pi^{2}\right) e^{-\Pi \mathcal{D}^{1 / 2} h}\right) \\
+\frac{\phi \mathcal{D}^{1 / 2} B}{4(1+B h)}\left[\left(\Pi \mathcal{D}^{1 / 2} h-2\right) e^{\Pi \mathcal{D}^{1 / 2} h}+2 \Pi \mathcal{D}^{1 / 2} h+\left(\Pi \mathcal{D}^{1 / 2} h+2\right) e^{-\Pi \mathcal{D}^{1 / 2} h}\right] \\
+\frac{\phi \mathcal{D}^{1 / 2}}{4 h(1+B h)}\left[\left(\Pi^{2} \mathcal{D} h^{2}-2\right)\left(e^{\Pi \mathcal{D}^{1 / 2} h}-e^{-\Pi \mathcal{D}^{1 / 2} h}\right)+4 \Pi \mathcal{D}^{1 / 2} h\right]
\end{gathered}
$$

and

$$
\begin{gathered}
I_{2}=-\frac{\mathcal{D}^{3 / 2} \phi}{8(1+B h) h}\left(e^{2 \Pi \mathcal{D}^{1 / 2} h}-e^{-2 \Pi \mathcal{D}^{1 / 2} h}\right)\left[\Pi \mathcal{D}^{1 / 2} h^{2}\left(\Pi \mathcal{D}^{1 / 2}+B\right)-2(1+B h)\right] \\
-\frac{\Pi \mathcal{D}^{2} \phi}{4(1+B h)}\left(1+e^{-2 \Pi \mathcal{D}^{1 / 2} h}\right)\left(e^{\Pi \mathcal{D}^{1 / 2} h}(2+B h)+B h\right) .
\end{gathered}
$$

Now as $\mathcal{D} \rightarrow 0$ we find that $I_{1} \rightarrow-\Pi^{2} \mathcal{D}+\mathcal{O}\left(\mathcal{D}^{2}\right)$ and $I_{2} \rightarrow \mathcal{O}\left(\mathcal{D}^{3}\right)$. Hence, in this limit we have, $I_{1} r_{2}+1 \simeq 0$. Thus, we retrieve the same limit that $r_{2} \rightarrow \Pi^{-2} \mathcal{D}^{-1}$. Further, as $\mathcal{D} \rightarrow \infty$ we find that

$$
I_{1} \rightarrow \frac{\mathcal{D}^{3 / 2} e^{\Pi \mathcal{D}^{1 / 2} h}}{4(1+B h)}\left(\phi \Pi^{2} h+\mathcal{D}^{-1 / 2}(\phi \Pi B h-2(1+B h))\right)
$$

and

$$
I_{2} \rightarrow-\frac{\mathcal{D}^{5 / 2} \Pi h \phi}{8(1+B h)} e^{2 \Pi \mathcal{D}^{1 / 2} h}\left(\Pi+B \mathcal{D}^{-1 / 2}\right)
$$


Hence, in the limit $\mathcal{D} \rightarrow 0$ we have, $I_{2} r_{2}^{2}+I_{1} r_{2} \simeq 0$, and again we retrieve the appropriate limit, $r_{2} \rightarrow 2 \mathcal{D}^{-1} e^{-\Pi \mathcal{D}^{1 / 2} h}$.

We note that when the mass or heat transport equation is integrated using a weight function given by the concentration or the temperature test function, a quadratic equation for $r_{2}$ is still obtained and yields the same results in the extreme $\mathcal{D}$-limits. Although the resulting quadratic equation obtained using non-unity weight functions yields more complicated versions of $I_{1}$ and $I_{2}$ than the ones presented here, all three methods produce very similar results for the solution $r_{2}$.

\section{F.3. Integral-boundary-layer model}

Following the approach employed to the flat film solution we could approximate the concentration and temperature by,

$$
\begin{aligned}
a^{m} & =\left(\frac{\cosh (b)-\cosh (b \eta)}{\cosh (b)-1}\right) \alpha, \quad \text { where } \quad b=\Pi \mathcal{D}^{1 / 2} h \\
T^{m} & =\left(\frac{(1+B h)(1-\cosh (b \eta))+\eta[b \sinh (b \eta)+B h(\cosh (b \eta)-1)]}{b \sinh (b)+1-\cosh (b)}\right) \theta
\end{aligned}
$$

which are test functions satisfying all of the boundary conditions and which also give the appropriate limiting solutions for both slow and fast reactions. However, we still have a problem with the Arrhenius term. [Note for a slow reaction a Taylor expansion of the Arrhenius term in small $\mathcal{D}$ was employed and for a fast reaction the Arrhenius term was set equal to $\Pi^{2}$.] In $\S$ F.1 we showed that a very good approximation to the Arrhenius term is

$$
e^{\frac{\mathcal{E} r}{1+r}} \simeq \frac{1+\Pi r}{1+\Pi^{-1} r}
$$

where $\Pi=e^{\mathcal{E} / 2}$ when $\mathcal{E}$ is small and $r \geq 0$. Using this approximation we can rewrite the concentration and energy residuals as,

$$
\begin{aligned}
\tilde{R}_{a}^{m} & =\left(1+\mathcal{D} a^{m}\right)\left(1+\Pi \mathcal{D} \phi T^{m}\right) \\
& +\left(3 \operatorname{ReSc}\left[a_{t}^{m}+u^{(0)} a_{x}^{m}+v^{(0)} a_{y}^{m}\right]-a_{y y}^{m}\right)\left(1+\Pi^{-1} \mathcal{D} \phi T^{m}\right) \\
\tilde{R}_{T}^{m} & =-\left(1+\mathcal{D} a^{m}\right)\left(1+\Pi \mathcal{D} \phi T^{m}\right) \\
& +\left(3 \operatorname{RePr}\left[T_{t}^{m}+u^{(0)} T_{x}^{m}+v^{(0)} T_{y}^{m}\right]-T_{y y}^{m}\right)\left(1+\Pi^{-1} \mathcal{D} \phi T^{m}\right),
\end{aligned}
$$

which in fact should be appropriate in both the small and large $\mathcal{D}$ limits as well as in the $\mathcal{O}(1)$ case. The Galerkin approach can then be applied, however, due to the size of the resulting equations, they are not listed here. Instead, we give the simplest averaged equation, the concentration one, obtained from a Shkadov approach, i.e. by employing a weight function of unity:

$$
\begin{aligned}
0 & =3 \operatorname{ReSc}\left[\left(\frac{d_{1}}{b}+\frac{\phi \theta\left(d_{2}+B h d_{3}\right)}{\Pi^{3} h^{2}(b H-1)}\right) \alpha_{t}+\left(\frac{d_{4}}{b^{3}}+\frac{\phi \theta\left(d_{5}+B h d_{6}\right)}{\Pi^{3} h^{2} b(b H-1)}\right) \frac{q}{h} \alpha_{x}\right] \\
& +3 \operatorname{ReSc}\left[\left(\frac{d_{7}}{b^{3}}+\frac{\phi \theta\left(d_{8}+B h d_{9}\right)}{\Pi^{3} h^{2} b^{2}(b H-1)}\right) \frac{q_{x}}{h} \alpha+\left(\frac{d_{10}}{b^{3}}+\frac{\phi \theta\left(d_{11}+B h d_{12}\right)}{\Pi^{3} h^{2} b^{2}(b H-1)}\right) \frac{q h_{x}}{h^{2}} \alpha\right] \\
& +\frac{1}{2}+\frac{b H}{2 h^{2}} \alpha+\frac{b d_{1}}{h^{2} \Pi^{2}} \alpha+\frac{b \phi \theta\left(d_{13}+B h d_{14}\right)}{2 \Pi^{3} h^{4}(b H-1)}\left(8 h^{2} \Pi^{2}+\alpha b^{2}\left(1+H^{2}\right)\right)
\end{aligned}
$$

where the functions $d_{1}$ to $d_{14}$ are only functions of $b=\Pi \mathcal{D}^{1 / 2} h$. With the introduction of $H=\operatorname{coth}(b / 2)$, these functions can be expressed as polynomials up to sixth order in $b$ and up to fifth order in $H$, defined 
by:

$$
\begin{aligned}
& d_{1}=\frac{b}{4}\left(H^{2}+1\right)-\frac{1}{2} H, \quad d_{13}=\left(b^{2}-2\right) H+b\left(H^{2}-1\right), \quad d_{14}=H(b H-2) \\
& d_{2}=\frac{b}{16}\left(2 b^{2} H\left(H^{2}+1\right)+b\left(3 H^{4}-10 H^{2}-1\right)-2 H\left(3 H^{2}-5\right)\right) \\
& d_{3}=\frac{b^{2}}{16}\left(3 H^{4}+1\right)-\frac{3 b}{8} H\left(H^{2}+1\right)+\frac{1}{2} \\
& d_{4}=\frac{b^{3}}{4}\left(H^{2}+1\right)-\frac{3 b^{2}}{4} H+\frac{3 b}{4}\left(1-H^{2}\right)+\frac{3}{2} H \\
& d_{5}=\frac{5 b^{4}}{32} H\left(H^{2}+1\right)+\frac{b^{3}}{16}\left(3 H^{4}-14 H^{2}-1\right)+\frac{3 b^{2}}{16}\left(3-H^{2}\right)-\frac{3 b}{64}\left(15 H^{4}-46 H^{2}-1\right) \\
& +\frac{3}{32} H\left(15 H^{2}-49\right) \\
& d_{6}=\frac{b^{4}}{32}\left(6 H^{4}+H^{2}+3\right)-\frac{9 b^{3}}{16} H\left(H^{2}+1\right)-\frac{3 b^{2}}{64}\left(15 H^{4}-22 H^{2}-9\right)+\frac{45 b}{32} H\left(H^{2}+1\right)-\frac{9}{2} \\
& d_{7}=\frac{b^{4}}{4} H\left(H^{2}-1\right)-\frac{b^{3}}{4}\left(H^{2}-1\right)-\frac{3 b^{2}}{4} H-\frac{3 b}{4}\left(H^{2}-1\right)+\frac{3}{2} H \\
& d_{8}=\frac{b^{4}}{32}\left(4 b^{2} H^{2}\left(H^{2}-1\right)+2 b H\left(3 H^{4}-10 H^{2}+7\right)-\left(9 H^{4}+10 H^{2}+5\right)\right) \\
& +\frac{3 b}{128}\left(4 b^{2} H\left(5 H^{2}+13\right)-b\left(15 H^{4}-94 H^{2}-15\right)+10 H\left(3 H^{2}-29\right)\right) \\
& d_{9}=\frac{b^{5}}{16} H\left(3 H^{4}-4 H^{2}+1\right)-\frac{3 b^{4}}{32}\left(3 H^{4}-2 H^{2}-1\right)-\frac{9 b^{3}}{32} H\left(H^{2}+1\right) \\
& -\frac{3 b^{2}}{128}\left(15 H^{4}-62 H^{2}-17\right)+\frac{45}{64} b H\left(H^{2}+1\right)-6 \\
& d_{10}=-\frac{b^{4}}{4} H\left(H^{2}-1\right)+\frac{3 b^{3}}{8}\left(H^{2}-1\right)+\frac{3 b^{2}}{4} H^{3}+\frac{3 b}{4}\left(H^{2}-1\right)-\frac{9}{2} H \\
& d_{11}=\frac{b^{4}}{64}\left(-10 b^{2} H^{2}\left(H^{2}-1\right)-12 b H\left(H^{4}-4 H^{2}+3\right)+3\left(H^{4}+10 H^{2}+5\right)\right) \\
& +\frac{3 b}{128}\left(2 b^{2} H\left(15 H^{4}-56 H^{2}+5\right)-b\left(15 H^{4}+95 H^{2}-13\right)-10 H\left(9 H^{2}-87\right)\right) \\
& d_{12}=-\frac{b^{5}}{32} H\left(6 H^{4}-7 H^{2}+1\right)+\frac{9 b^{4}}{64}\left(3 H^{4}-2 H^{2}-1\right)+\frac{3 b^{3}}{64} H\left(15 H^{4}-32 H^{2}+29\right) \\
& -\frac{3 b^{2}}{128}\left(15 H^{4}+31 H^{2}-13\right)-\frac{135}{64} b H\left(H^{2}+1\right)+18 .
\end{aligned}
$$

\section{G. Validity check of the temperature and concentration fields used in IBL}

To check the validity of the temperature and concentration fields used in our IBL models. We follow [24] and carry out a computation of the first-order heat and mass transport equations for $\mathcal{M}=0$ using the fluid flow obtained from IBL. The reason for this is two-fold: (i) in this limit the hydrodynamics is not affected by the concentration and temperature which then reduces substantially the computational effort; (ii) we know from previous studies on free falling films (e.g. [2]) that the averaged momentum equation (6.13) (for $\mathcal{M}=0$ ) provides a proper description of the hydrodynamics. Substituting then the 

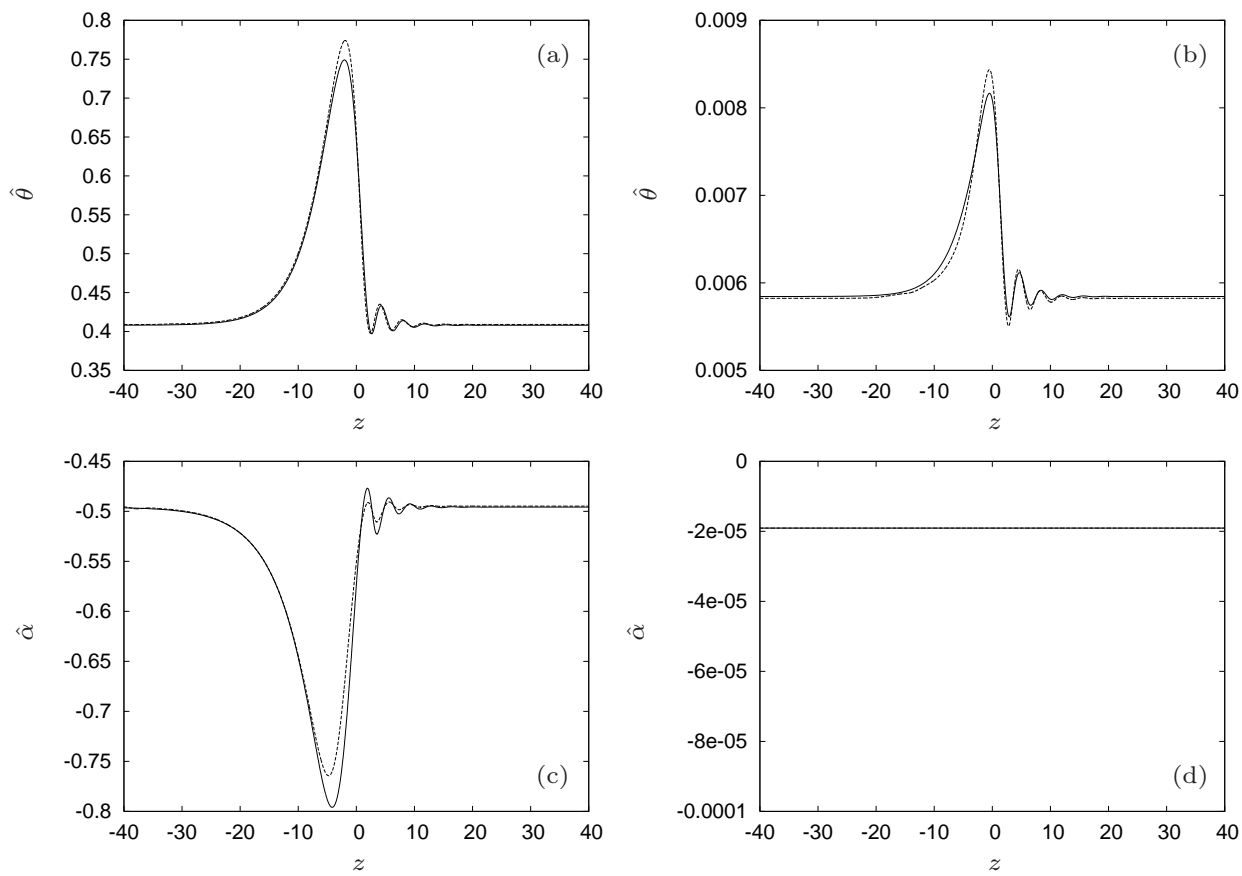

FiguRE 10. Comparison of the surface temperature $\hat{\theta}$ and wall concentration $\hat{\alpha}$ wave profiles for $\mathcal{M}=0$. The solid lines are computed with the IBL models while the broken lines are obtained from the finite-differencing scheme. The dimensionless groups are $\Gamma=3000, \mathrm{Bi}=0.1, \operatorname{Pr}=7, \mathrm{Sc}=7, \mathcal{E}=1, \phi=1$, and $\beta=\pi / 2 .-(\mathrm{a})$ and (c): small Da case; $\mathrm{Re}=4$ and $\mathrm{Da}=0.01-(\mathrm{b})$ and $(\mathrm{d})$ : large Da case; $\mathrm{Re}=4$ and $\mathrm{Da}=10000$. The concentration curves in this case are practically indistinguishable.

flow field from (6.10) into the heat and mass transport equations (6.14), we obtain

$$
\begin{aligned}
a_{\eta \eta} & =\frac{3 \mathrm{ScRe}}{2 \mathrm{We}^{1 / 3}}\left[\left(3 q h\left(2 \eta-\eta^{2}\right)-2 c h^{2}\right) a_{z}+c h h_{z} \eta(\eta-1)(\eta-2) a_{\eta}\right] \\
& +h^{2}(1+\mathcal{D} a) e^{\frac{\mathcal{E} \phi \phi T}{1+\mathcal{D} \phi T}} \\
T_{\eta \eta} & =\frac{3 \operatorname{PrRe}}{2 \mathrm{We}^{1 / 3}}\left[\left(3 q h\left(2 \eta-\eta^{2}\right)-2 c h^{2}\right) T_{z}+c h h_{z} \eta(\eta-1)(\eta-2) T_{\eta}\right] \\
& -h^{2}(1+\mathcal{D} a) e^{\frac{\mathcal{E} \phi T T}{1+\mathcal{D} \phi T}}
\end{aligned}
$$

subject to the boundary conditions,

$$
\begin{array}{rll}
a_{\eta}=T=0 & \text { on } & \eta=0 \\
a=T_{\eta}+B h T=0 & \text { on } & \eta=1 .
\end{array}
$$

The above system is solved numerically in two dimensions along with periodic boundary conditions in the $z$-direction using a finite-differences scheme. The film thickness $h$ is obtained from the isothermal momentum equation (6.13) in the moving frame $z$ with $q=\frac{1}{3}+c(h-1)$. Profiles of the wall concentration $\hat{\alpha}$ and surface temperature $\hat{\theta}$ constructed this way for typical values of the dimensionless groups are depicted in figure 10 and compared to those obtained with the IBL equations (for brevity we drop the superscripts $s$ and $f$ corresponding to small and large Da, respectively). They show that the test functions used in the IBL models to account for the concentration and temperature fields in the film allow for an accurate 
description of the nonlinear regime for $\hat{\alpha}$ and $\hat{\theta}$. The agreement is very good in both the small- and large-Da limits. In the latter case, the wall concentration depicted in figure $10 \mathrm{~d}$ is uniform and close to zero since, as the chemical reaction is fast, the reactant is consumed before it can reach the wall. In figure 10b, the small shift observed in the profile flat region is due to the Arrhenius term approximation: finite-difference calculations with a constant Arrhenius term yield a profile closer to the one obtained with the IBL model.

\section{References}

[1] A. Oron, S. H. Davis, S. G. Bankoff. Long-scale evolution of thin liquid films. Rev. Mod. Phys., 69 (1997), 931-980.

[2] S. Kalliadasis, U. Thiele (Ed) Thin Films of Soft Matter. Springer-Wien, New York, 2007.

[3] R. V. Craster, O. K. Matar. Dynamics and stability of thin liquid films. Rev. Mod. Phys., 81 (2009), $1131-1198$.

[4] P. L. Kapitza. Wave flow of thin layers of viscous fluid: I. Free flow. Zh. Eksp. Tear. Fiz., 18 (1948), 3-18.

[5] P. L. Kapitza. Wave flow of thin layers of a viscous fluid: II. Fluid flow in the presence of continuous gas flow and heat transfer. Zh. Eksp. Teor. Fiz., 18 (1948), 19-28.

[6] P. L. Kapitza, S. P. Kapitza. Wave flow of thin layers of a viscous fluid: III. Experimental study of undulatory flow conditions. Zh. Eksp. Teor. Fiz., 19 (1949), 105-120.

[7] H.-C. Chang. Wave evolution on a falling film. Ann. Rev. Fluid Mech., 26 (1994), 103-136.

[8] H.-C. Chang, E. A. Demekhin. Complex Wave Dynamics on Thin Films. Elsevier, 2002.

[9] Z. Dagan, L. M. Pismen. Marangoni waves induced by a multistable chemical reaction on thin liquid films. J. Colloid Interface Sci., 99 (1984), 215-225.

[10] A. Pereira, P. M. J. Trevelyan, U. Thiele, S. Kalliadasis. Interfacial hydrodynamic waves driven by chemical reactions. J. Eng. Math., 59 (2007), 207-220.

[11] A. Pereira, P. M. J. Trevelyan, U. Thiele, S. Kalliadasis. Dynamics of a horizontal thin liquid film in the presence of reactive surfactants. Phys. Fluids, 19 (2007), 112102.

[12] A. Pereira, P. M. J. Trevelyan, U. Thiele, S. Kalliadasis. Interfacial instabilities driven by chemical reactions. Eur. Phys. J. Special Topics, 166 (2009), 121-125.

[13] A. Y. Rednikov, Y. S. Ryazantsev, M. G. Velarde. Drop motion with surfactant transfer in a homogeneous surrounding. Phys. Fluids, 6 (1994), 451-468.

[14] L. M. Pismen. Chemocapillary instabilities of a contact line. Phys. Rev. E, 81 (2010), 026307.

[15] E. R. Gilliland, R. F. Baddour, P. L. T. Brian. Gas Absorption Accompanied by a Liquid-phase Chemical Reaction. Am. Inst. Chem. Eng. J., 4 (1958), 223.

[16] P. M. J. Trevelyan, S. Kalliadasis. Dynamics of a reactive falling film at large Péclet numbers. I. Long-wave approximation. Phys. Fluids, 16 (2004), 3191-3208.

[17] P. M. J. Trevelyan, S. Kalliadasis. Dynamics of a reactive falling film at large Péclet numbers. II. Nonlinear waves far from criticality: Integral-boundary-layer approximation. Phys. Fluids, 16 (2004), 3209-3226.

[18] A. A. Nepomnyashchy, M. G. Velarde, P. Colinet. Interfacial Phenomena and Convection. Chapman \& Hall, London, 2002.

[19] R. C. Weast, M. J. Astle. Handbook of Chemistry and Physics. CRC Press, Boca Raton, FL, 1979.

[20] C. E. Wylock, P. Colinet, T. Cartage, B. Haut. Coupling between mass transfer and chemical reactions during the absorption of $\mathrm{CO}_{2}$ in a $\mathrm{NaHCO}_{3}-\mathrm{Na}_{2} \mathrm{HCO}_{3}$ brine: Experimental and theoretical study. Int. J. Chem. React. Engng., 6 (2008), A4.

[21] S. Kalliadasis, E. A. Demekhin, C. Ruyer-Quil, M. G. Velarde. Thermocapillary instability and wave formation on a film falling down a uniformly heated plane. J. Fluid Mech., 492 (2003), 303-338.

[22] P. Trevelyan, S. Kalliadasis. Wave dynamics on a thin-liquid film falling down a heated wall. J. Eng. Math., 50 (2004), 177-208.

[23] C. Ruyer-Quil, B. Scheid, S. Kalliadasis, M. G. Velarde, R. Kh. Zeytounian. Thermocapillary long waves in a liquid film flow. Part 1. Low-dimensional formulation. J. Fluid Mech., 538 (2005), 199-222.

[24] P. M. J. Trevelyan, B. Scheid, C. Ruyer-Quil, S. Kalliadasis. Heated falling films. J. Fluid Mech., 592 (2007), $295-334$.

[25] T. B. Benjamin. Wave formation in laminar flow down an inclined plane. J. Fluid Mech., 2 (1957), 554-574.

[26] C. S. Yih. Stability of liquid flow down an inclined plane. Phys. Fluids, 6 (1963), 321-334.

[27] D. J. Benney. Long waves on liquid films. J. Math. Phys., 45 (1966), 150-155.

[28] B. Scheid, C. Ruyer-Quil, S. Kalliadasis, M. G. Velarde, R. Kh. Zeytounian. Thermocapillary long waves in a liquid film flow. Part 2. Linear stability and nonlinear waves. J. Fluid Mech., 538 (2005), 223-244.

[29] C. Ruyer-Quil, P. Trevelyan, F. Giorgiutti-Dauphiné, C. Duprat, S. Kalliadasis. Modelling film flows down a fibre. J. Fluid Mech., 603 (2008), 431-462.

[30] A. Pumir, P. Manneville, Y. Pomeau. On solitary waves running down an inclined plane. J. Fluid Mech., 135 (1983), 27-50.

[31] C. Nakaya. Waves on a viscous fluid film down a vertical wall. Phys. Fluids, 1 (1989), 1143-1154.

[32] A. Oron, O. Gottlieb. Nonlinear dynamics of temporally excited falling liquid films. Phys. Fluids, 14 (2002), 2622-2636. 
[33] B. Scheid, C. Ruyer-Quil, U. Thiele, O.A. Kabov, J.C. Legros, P. Colinet. Validity domain of the Benney equation including Marangoni effect for closed and open flows. J. Fluid Mech., 527 (2004), 303-335.

[34] V. Ya. Shkadov. Wave modes in the flow of thin layer of a viscous liquid under the action of gravity. Izv. Akad. Nauk SSSR, Mekh. Zhidk Gaza, 1 (1967), 43-50.

[35] C. Ruyer-Quil, P. Manneville. Modeling film flows down inclined planes. Eur. Phys. J. B, 6 (1998), $277-292$.

[36] C. Ruyer-Quil, P. Manneville. Improved Modeling of flows down inclined planes. Eur. Phys. J. B, 15 (2000), $357-369$.

[37] C. Ruyer-Quil, P. Manneville. Further accuracy and convergence results on the modeling of flows down inclined planes by weighted-residual approximations. Phys. Fluids, 14 (2002), 170-183.

[38] S. Kalliadasis, A. Kiyashko, E. A. Demekhin. Marangoni instability of a thin liquid film heated from below by a local heat source. J. Fluid Mech., 475 (2003), 377-408.

[39] I. L. Kliakhandler, S. H. Davis, S. G. Bankoff. Viscous beads on vertical fibre. J. Fluid Mech., 429 (2001), 381-390.

[40] C. Duprat, C. Ruyer-Quil, S. Kalliadasis, F. Giorgiutti-Dauphiné. Absolute and convective instabilities of a viscous film flowing down a vertical fiber. Phys. Rev. Lett., 98 (2007), 244502.

[41] N. A. Malamataris, M. Vlachogiannis, V. Bontozoglou. Solitary waves on inclined films: Flow structure and binary interactions. Phys. Fluids, 14 (2002), 1082-1094.

[42] B. Scheid, C. Ruyer-Quil, P. Manneville. Wave patterns in film flows: modelling and three-dimensional waves. J. Fluid Mech., 562 (2006), 183-222.

[43] E. Doedel, A. Champneys, T. Fairfrieve, Y. Kuznetsov, B. Sandstede, X. Wang. AUTO97: Continuation and bifurcation software for ordinary differential equations. Concordia University, Montreal, 1997.

[44] P. Huerre, P. A. Monkewitz. Local and global instabilities in spatially developing flows. Annu. Rev. Fluid. Mech., 22 (1990), 473-537.

[45] P. Huerre, M. Rossi. Hydrodynamic and Nonlinear Instabilities. In: Hydrodynamic Instabilities in Open Flows (Ed. C. Godréche, P. Manneville), Cambridge University Press, 1998, 81-294.

[46] H.-C. Chang, E. A. Demekhin, D. I. Kopelevich. Stability of a solitary pulse against wave packet disturbances in an active medium. Phys. Rev. Lett., 75 (1995), 1747-1750.

[47] E. A. Demekhin, E. N. Kalaidin, S. Kalliadasis, S. Yu. Vlaskin. Three-dimensional localized coherent structures of surface turbulence. II. A solitons., Phys. Fluids, 19 (2007), 114104.

[48] A. A. Golovin, A. A. Nepomnyashchy, L. M. Pismen. Interaction between short-scale Marangoni convection and longscale deformation instability. Phys. Fluids, 6 (1994), 34-48.

[49] U. Thiele, E. Knobloch. Thin liquid films on a slightly inclined heated plate. Physica D, 190 (2004), 213-248. 